\title{
BIVARIANCE, GROTHENDIECK DUALITY AND HOCHSCHILD HOMOLOGY I: CONSTRUCTION OF A BIVARIANT THEORY*
}

\author{
LEOVIGILDO ALONSO TARRÍO ${ }^{\dagger}$, ANA JEREMÍAS LÓPEZ AND JOSEPH LIPMAN $^{\S}$
}

To Heisuke Hironaka, on the occasion of his 80th birthday

\begin{abstract}
A procedure for constructing bivariant theories by means of Grothendieck duality is developed. This produces, in particular, a bivariant theory of Hochschild (co)homology on the category of schemes that are flat, separated and essentially of finite type over a noetherian scheme $S$. The theory takes values in the category of symmetric graded modules over the graded-commutative ring $\oplus_{i} \mathrm{H}^{i}\left(S, \mathcal{O}_{S}\right)$. In degree $i$, the cohomology and homology $\mathrm{H}^{0}\left(S, \mathcal{O}_{S}\right)$-modules thereby associated to such an $x: X \rightarrow S$, with Hochschild complex $\mathcal{H}_{x}$, are $\operatorname{Ext}_{\mathcal{O}_{X}}^{i}\left(\mathcal{H}_{x}, \mathcal{H}_{x}\right)$ and $\operatorname{Ext}_{\mathcal{O}_{X}}^{-i}\left(\mathcal{H}_{x}, x^{!} \mathcal{O}_{S}\right)(i \in \mathbb{Z})$. This lays the foundation for a sequel that will treat orientations in bivariant Hochschild theory through canonical relative fundamental class maps, unifying and generalizing previously known manifestations, via differential forms, of such maps.
\end{abstract}

Key words. Hochschild homology, bivariant, Grothendieck duality, fundamental class.

AMS subject classifications. Primary 14F99.

Introduction. Grothendieck duality is a cornerstone of cohomology theory for quasi-coherent sheaves in Algebraic Geometry. It relates the classical theory of the canonical linear system of a variety to an analogue of Poincaré duality. Indeed, one of the outstanding features of Grothendieck duality is the interplay between concrete and abstract aspects of the theory, the former being expressed in terms of differentials and residues, while the latter are conveyed in terms of a formalism of certain functors between derived categories - the Grothendieck operations, and a web of relations among them (see, e.g., [L3]). These two aspects are linked by the fundamental class of a scheme-map.

In its usual incarnation the fundamental class is, for a noetherian-scheme map $x: X \rightarrow S$ that is separated, essentially finite type, perfect (i.e., of finite flat dimension or finite tor-dimension), and equidimensional of relative dimension $n$, a canonical derived-category map from suitably shifted top-degree relative differentials to the relative dualizing complex:

$$
C_{X \mid S}: \Omega_{X \mid S}^{n}[n] \rightarrow x^{!} \mathcal{O}_{S}
$$

where $x^{!}$is the twisted inverse image functor which is the principal actor in Grothendieck duality theory; or equivalently, a map of coherent sheaves

$$
c_{X \mid S}: \Omega_{X \mid S}^{n} \rightarrow \omega_{X \mid S}:=H^{-n} x^{!} \mathcal{O}_{S},
$$

where $\omega_{X \mid S}$ is the relative dualizing (or canonical) sheaf associated to $x$.

*Received May 24, 2010; accepted for publication May 20, 2011. Authors partially supported by Spain's MICIIN and E.U.'s FEDER research project MTM2008-03465. Third author also partially supported over time by NSF and NSA.

†Departamento de Álxebra, Facultade de Matemáticas, Universidade de Santiago de Compostela, E-15782 Santiago de Compostela, Spain (leo.alonso@usc.es).

‡Departamento de Álxebra, Facultade de Matemáticas, Universidade de Santiago de Compostela, E-15782 Santiago de Compostela, Spain (ana.jeremias@usc.es).

$\S$ Department of Mathematics, Purdue University, West Lafayette IN 47907, USA (lipman@math. purdue.edu). 
In case $x$ is a smooth map, $c_{X \mid S}$ is the isomorphism that is well-known from Serre duality. More general situations have been studied in various contexts, local and global, algebraic and analytic, e.g., [AnZ], [An], [AnL], [KW], [Kd]. In [L1], there is a concrete treatment of the case when $S=\operatorname{Spec}(k)$ with $k$ a perfect field and $X$ an integral algebraic scheme over $k$. The map $c_{X \mid k}$ is realized there as a globalization of the local residue maps at the points of $X$, leading to explicit versions of local and global duality and the relation between them. These results are generalized to certain maps of noetherian schemes in [HS]. In all these approaches, an important role is played - via factorizations of $x$ as smooth $\circ$ finite - by the case $n=0$, where the notion of fundamental class is equivalent to that of traces of differential forms.

After [L2] it became clear that Hochschild homology and cohomology play a role in this circle of ideas. The connection with differentials comes via canonical maps from differential forms to sheafified Hochschild homology.

Over schemes, the theory of Hochschild homology and cohomology goes back to work of Gerstenhaber and Schack [GhS] on deformation problems, see [BF1], [GeW], $[\mathrm{Ca} 2]$ and $[\mathrm{CaW}]$. Recently, more refined versions of the theory have been developed, in $[\mathrm{BF} 1]$ and $[\mathrm{LV}]$.

Our first main task is to construct, over a fixed noetherian base scheme $S$, a bivariant theory $[\mathrm{FM}]$, taking values in derived categories of complexes with quasicoherent homology, those categories being enriched by graded modules over the graded-commutative ring $H:=\bigoplus_{i \in \mathbb{Z}} H^{i}\left(S, \mathcal{O}_{S}\right)$.

The construction makes use of properties of the Hochschild complex $\mathcal{H}_{x}$ of a separated, essentially finite-type, perfect map $x: X \rightarrow S$ - that is, the derived-category object $\mathrm{L} \delta^{*} \mathrm{R} \delta_{*} \mathcal{O}_{X}$ where $\delta: X \rightarrow X \times_{S} X$ is the diagonal map - and on basic facts from Grothendieck duality theory. (Strictly speaking, this $\mathcal{H}_{x}$ should be called the "Hochschild complex" only when $x$ is flat.) The $H$-module thereby associated to a morphism $f:(X \stackrel{x}{\rightarrow} S) \rightarrow(Y \stackrel{y}{\rightarrow} S)$ of such $S$-schemes is

$$
\mathrm{HH}^{*}(f):=\oplus_{i \in \mathbb{Z}} \operatorname{Ext}_{\mathcal{O}_{X}}^{i}\left(\mathcal{H}_{x}, f^{!} \mathcal{H}_{y}\right)=\oplus_{i \in \mathbb{Z}} \operatorname{Hom}_{\mathbf{D}(X)}\left(\mathcal{H}_{x}, f^{!} \mathcal{H}_{y}[i]\right)
$$

so that the associated cohomology groups are

$$
\mathrm{HH}^{i}(X \mid S):=\operatorname{HH}^{i}\left(\operatorname{id}_{X}\right)=\operatorname{Ext}_{\mathcal{O}_{X}}^{i}\left(\mathcal{H}_{x}, \mathcal{H}_{x}\right)
$$

and the associated homology groups are

$$
\mathrm{HH}_{i}(X \mid S):=\mathrm{HH}^{-i}(x)=\operatorname{Ext}_{\mathcal{O}_{X}}^{-i}\left(\mathcal{H}_{x}, x^{!} \mathcal{O}_{S}\right)
$$

Over smooth $\mathbb{C}$-schemes, these bivariant homology groups have been studied in [Ca1], and in more sophisticated terms, in $[\mathrm{CaW}]$. The bivariant cohomology groups form a graded algebra, of which the cohomology algebra in [Ca1] is an algebra retract. (These bivariant groups are not to be confused with the bivariant cohomology groups in $[$ Lo, $§ 5.5 .1]$.)

The data constituting the bivariant theory are specified in section 3, and satisfaction of the bivariant axioms is verified in section 4 . The construction is organized around purely category-theoretic properties of the derived direct- and inverse-image pseudofunctors, and of the twisted inverse image pseudofunctor (section 2), and of $\mathcal{H}_{x}$ (section 3). This makes it applicable in other contexts where duality theory exists, such as noetherian formal schemes or certain maps of nonnoetherian schemes. Moreover, the simple properties of $\mathcal{H}_{x}$ that are needed are shared, e.g., by the cotangent complex of $x$, or by the "true" Hochschild complex in [BF1]. 
Section 5 is devoted to showing that the formal properties in section 2 do come out of Grothendieck duality for separated essentially-finite-type perfect maps of noetherian schemes. It is only recently that duality theory has been made available for essentially-finite-type, rather than just finite-type, maps (see [Nk2]), making possible a unified treatment of local and global situations. That theory requires the tedious verification of commutativity of a multitude of diagrams, and more of the same is needed for our purposes. That accounts in part for the length of section 5 ; but there is more to be checked, for example because of the upgrading of results about derived categories to the $H$-graded context. Thus the bivariant Hochschild theory, though quickly describable, as above, encompasses many relations.

To put the present results in context, let us discuss very briefly our second main task, to be carried out in the sequel to this paper-namely, to develop the notion of the fundamental class of an $f$ as above. This is an element

$$
\varrho(f):=\mathbf{c}_{f}\left(\mathcal{O}_{Y}\right) \in \operatorname{HH}^{0}(f) .
$$

In particular, when $y=\mathrm{id}_{S}$, one gets a map in $\mathrm{HH}^{0}(x)=\mathrm{HH}_{0}(X \mid S)$,

$$
\varrho(x): \mathcal{H}_{x} \rightarrow x^{!} \mathcal{O}_{S},
$$

which together with a natural map $\Omega_{X \mid S}^{i} \rightarrow H^{-i} \mathcal{H}_{x}$ gives a map

$$
\Omega_{X \mid S}^{i} \rightarrow H^{-i} x^{!} \mathcal{O}_{S} \quad(i \geq 0),
$$

that generalizes (0.0.1) when $x$ is flat, separated, and essentially finite type.

Two basic properties of the fundamental class are:

1) Transitivity vis-à-vis a composite map of $S$-schemes $X \stackrel{u}{\rightarrow} Y \stackrel{v}{\rightarrow} Z$, i.e.,

$$
\mathbf{c}_{v u}=u ! \mathbf{c}_{v} \circ \mathbf{c}_{u} v^{*} .
$$

2) Compatibility with essentially étale base change.

Transitivity gives in particular that $\mathbf{c}_{v u}\left(\mathcal{O}_{Z}\right)=u^{!} \mathbf{c}_{v}\left(\mathcal{O}_{Z}\right) \circ \mathbf{c}_{u}\left(\mathcal{O}_{Y}\right)$. In terms of the bivariant product $\mathrm{HH}^{0}(u) \times \mathrm{HH}^{0}(v) \rightarrow \mathrm{HH}^{0}(v u)$, this says:

$$
\varrho(v u)=\varrho(u) \cdot \varrho(v) .
$$

Thus the family $\varrho(f)$ is a family of canonical orientations, compatible with essentially étale base change, for the flat maps in our bivariant theory [FM, p. 28, 2.6.2].

With this in hand, one can apply the general considerations in [FM] to obtain, for example, Gysin morphisms, that provide "wrong-way" functorialities for homology and cohomology.

1. Review of graded categories and functors. Let there be given a gradedcommutative ring $H=\oplus_{i \in \mathbb{Z}} H^{i}$,

$$
h h^{\prime}=(-1)^{m n} h^{\prime} h \in H^{m+n} \quad\left(h \in H^{n}, h^{\prime} \in H^{m}\right) .
$$

We will use the language of $H$-graded categories. So let us recall some of the relevant basic notions.

1.1. A category $\mathbf{E}$ is $H$-graded if

(i) for any objects $A, B$ in $\mathbf{E}$, the set $\mathbf{E}(A, B)$ of arrows from $A$ to $B$ is equipped with a symmetric graded $H$-module structure: $\mathbf{E}(A, B)$ is a graded abelian group

$$
\mathbf{E}(A, B)=\oplus_{i \in \mathbb{Z}} \mathbf{E}^{i}(A, B)
$$


with both left and right graded $H$-module structures such that

$$
h \alpha=(-1)^{m n} \alpha h \quad\left(h \in H^{n}, \alpha \in \mathbf{E}^{m}(A, B)\right),
$$

(so each of these structures determines the other); and further,

(ii) for any $C \in \mathbf{E}$, the composition map $\mathbf{E}(B, C) \times \mathbf{E}(A, B) \stackrel{\circ}{\longrightarrow} \mathbf{E}(A, C)$ is graded $H$-bilinear: it is $\mathbb{Z}$-bilinear, and such that for $\beta \in \mathbf{E}^{m}(B, C), \alpha \in \mathbf{E}^{n}(A, B), h \in H$, it holds that $\beta \circ \alpha \in \mathbf{E}^{m+n}(A, C)$ and

$$
(h \beta) \circ \alpha=h(\beta \circ \alpha), \quad \beta \circ(\alpha h)=(\beta \circ \alpha) h .
$$

It follows that $(\beta h) \circ \alpha=\beta \circ(h \alpha)$, and then that composition factors uniquely through a homomorphism of symmetric graded $H$-modules

$$
\mathbf{E}(B, C) \otimes_{H} \mathbf{E}(A, B) \rightarrow \mathbf{E}(A, C) .
$$

Any full subcategory of an $H$-graded category $\mathbf{E}$ is naturally $H$-graded.

1.1.1. For any object $A$ in an $H$-graded category $\mathbf{E}, \mathbf{E}(A, A)$ has a natural graded $H$-algebra structure. Indeed, the identity id ${ }_{A}$, being idempotent, is in $\mathbf{E}^{0}(A, A)$, and the map $\tau_{A}: H \rightarrow \mathbf{E}(A, A)$ such that for all $n$ and $h \in H^{n}$,

$$
\tau_{A}(h)=h \operatorname{id}_{A}=\operatorname{id}_{A} h \in \mathbf{E}^{n}(A, A)
$$

is a graded-ring homomorphism - since

$$
\left(h \operatorname{id}_{A}\right) \circ\left(h^{\prime} \operatorname{id}_{A}\right)=h\left(\operatorname{id}_{A} \circ\left(\operatorname{id}_{A} h^{\prime}\right)\right)=h\left(\left(\operatorname{id}_{A} \circ \operatorname{id}_{A}\right) h^{\prime}\right)=h h^{\prime} \operatorname{id}_{A}-
$$

that maps $H$ to the graded center of $\mathbf{E}(A, A)$ - that is, for $\alpha \in \mathbf{E}^{m}(A, A)$,

$$
\left(h \operatorname{id}_{A}\right) \circ \alpha=h\left(\operatorname{id}_{A} \circ \alpha\right)=h \alpha=(-1)^{m n}(\alpha h) \circ \operatorname{id}_{A}=(-1)^{m n} \alpha \circ\left(h \operatorname{id}_{A}\right) .
$$

1.1.2. A preadditive category is an $H$-graded category with $H=\oplus_{i \in \mathbb{Z}} H^{i}$, the graded ring such that $H^{0}=\mathbb{Z}$ and $H^{i}=(0)$ for all $i \neq 0$.

1.2. Let $\mathbf{E}_{1}$ and $\mathbf{E}_{2}$ be $H$-graded categories. A functor $F: \mathbf{E}_{1} \rightarrow \mathbf{E}_{2}$ is said to be $H$-graded if the maps $\mathbf{E}_{1}(A, B) \rightarrow \mathbf{E}_{2}(F A, F B)\left(A, B \in \mathbf{E}_{1}\right)$ associated to $F$ are graded $H$-linear.

Another $H$-graded functor $G$ being given, a functorial map $\xi: F \rightarrow G$ of degree $n$ is a family of arrows $\xi_{A} \in \mathbf{E}_{2}^{n}(F A, G A)\left(A \in \mathbf{E}_{1}\right)$ such that for any $\alpha \in \mathbf{E}_{1}^{m}(A, B)$, it holds that $(G \alpha) \circ \xi_{A}=(-1)^{m n} \xi_{B} \circ(F \alpha)$; in other words, the following diagram commutes up to the sign $(-1)^{m n}$ :

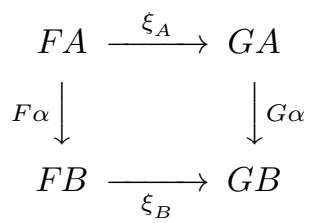

Composing a functorial map of degree $n_{1}$ with one of degree $n_{2}$ produces one of degree $n_{1}+n_{2}$. 
1.3. The graded center $\mathcal{C}=\mathcal{C}_{\mathbf{E}}$ of an $H$-graded category $\mathbf{E}$ is, to begin with, the graded abelian group whose $n$-th degree homogeneous component $\mathcal{C}^{n}$ consists of the degree- $n$ self-maps of the identity functor id $\mathbf{E}$ of $\mathbf{E}$.

This $\mathcal{C}_{\mathbf{E}}$ does not change when $H$ is replaced by the trivially graded ring $\mathbb{Z}$.

Composition of functorial maps gives a product

$$
\mathcal{C}^{m} \times \mathcal{C}^{n} \rightarrow \mathcal{C}^{m+n} \quad(m, n \in \mathbb{Z}),
$$

for which, evidently, if $\xi \in \mathcal{C}^{m}$ and $\zeta \in \mathcal{C}^{n}$ then $\xi \zeta=(-1)^{m n} \zeta \xi$. Hence $\mathcal{C}$ can be viewed, via the graded-ring homomorphism $\tau: H \rightarrow \mathcal{C}$ that takes $h \in H^{n}$ to the family $\tau_{A}(h)=h \operatorname{id}_{A} \in \mathbf{E}^{n}(A, A)(A \in \mathbf{E})$, as a graded-commutative graded $H$-algebra.

For $\xi \in \mathcal{C}^{n}$, composition with $\xi_{A}$ (resp. $\xi_{B}$ ) maps $\mathbf{E}^{m}(A, B)$ to $\mathbf{E}^{m+n}(A, B)$; this produces a symmetric graded $\mathcal{C}$-module structure on $\mathbf{E}(A, B)$. Hence the category $\mathbf{E}$ is $\mathcal{C}$-graded. The original $H$-grading is obtained from the $\mathcal{C}$-grading by restricting scalars via $\tau$.

In the case $H=\mathcal{C}$, the above map $\tau_{A}$ becomes the evaluation map

$$
\mathrm{ev}_{A}: \mathcal{C} \rightarrow \mathbf{E}(A, A)
$$

taking $\xi \in \mathcal{C}^{n}$ to the map $\xi_{A}$.

1.4. The tensor product $\mathbf{E}_{1} \otimes_{H} \mathbf{E}_{2}$ of $H$-graded categories is the $H$-graded category whose objects are pairs $\left(A_{1}, A_{2}\right)\left(A_{1} \in \mathbf{E}_{1}, A_{2} \in \mathbf{E}_{2}\right)$, and such that

$$
\left(\mathbf{E}_{1} \otimes_{H} \mathbf{E}_{2}\right)\left(\left(A_{1}, A_{2}\right),\left(B_{1}, B_{2}\right)\right):=\mathbf{E}_{1}\left(A_{1}, B_{1}\right) \otimes_{H} \mathbf{E}_{2}\left(A_{2}, B_{2}\right)
$$

with the obvious symmetric graded $H$-module structure, composition

$$
\begin{aligned}
\left(\mathbf{E}_{1}\left(B_{1}, C_{1}\right) \otimes_{H} \mathbf{E}_{2}\left(B_{2}, C_{2}\right)\right) \times\left(\mathbf{E}_{1}\left(A_{1}, B_{1}\right) \otimes_{H}\right. & \left.\mathbf{E}_{2}\left(A_{2}, B_{2}\right)\right) \\
& \longrightarrow \mathbf{E}_{1}\left(A_{1}, C_{1}\right) \otimes_{H} \mathbf{E}_{2}\left(A_{2}, C_{2}\right)
\end{aligned}
$$

being derived from the graded $H$-quadrilinear map

$$
\mathbf{E}_{1}\left(B_{1}, C_{1}\right) \times \mathbf{E}_{2}\left(B_{2}, C_{2}\right) \times \mathbf{E}_{1}\left(A_{1}, B_{1}\right) \times \mathbf{E}_{2}\left(A_{2}, B_{2}\right) \rightarrow \mathbf{E}_{1}\left(A_{1}, C_{1}\right) \otimes_{H} \mathbf{E}_{2}\left(A_{2}, C_{2}\right)
$$

such that for all $A_{1} \stackrel{\alpha_{1}}{\longrightarrow} B_{1} \stackrel{\beta_{1}}{\longrightarrow} C_{1}$ in $\mathbf{E}_{1}$ and $A_{2} \stackrel{\alpha_{2}}{\longrightarrow} B_{2} \stackrel{\beta_{2}}{\longrightarrow} C_{2}$ in $\mathbf{E}_{2}$, with $\alpha_{1} \in \mathbf{E}_{1}^{m_{1}}\left(A_{1}, B_{1}\right)$ and $\beta_{2} \in \mathbf{E}_{2}^{n_{2}}\left(B_{2}, C_{2}\right)$, it holds that

$$
\left(\beta_{1}, \beta_{2}, \alpha_{1}, \alpha_{2}\right) \mapsto(-1)^{n_{2} m_{1}}\left(\beta_{1} \circ \alpha_{1}\right) \otimes\left(\beta_{2} \circ \alpha_{2}\right) .
$$

In particular,

$$
\left(\beta_{1} \otimes \beta_{2}\right) \circ\left(\alpha_{1} \otimes \alpha_{2}\right)=(-1)^{n_{2} m_{1}}\left(\beta_{1} \circ \alpha_{1}\right) \otimes\left(\beta_{2} \circ \alpha_{2}\right): A_{1} \otimes A_{2} \rightarrow C_{1} \otimes C_{2} .
$$

1.4.1. Notation. Given $A_{k}, B_{k} \in \mathbf{E}_{k}, \alpha_{k} \in \mathbf{E}_{k}\left(A_{k}, B_{k}\right)(k=1,2)$, and a functor $\bar{\otimes}: \mathbf{E}_{1} \otimes_{H} \mathbf{E}_{2} \rightarrow \mathbf{E}$, set

$$
\begin{gathered}
A_{1} \bar{\otimes} A_{2}:=\bar{\otimes}\left(A_{1}, A_{2}\right), \\
\alpha_{1} \bar{\otimes} \alpha_{2}:=\bar{\otimes}\left(\alpha_{1} \otimes \alpha_{2}\right): A_{1} \bar{\otimes} A_{2} \rightarrow B_{1} \bar{\otimes} B_{2} .
\end{gathered}
$$


1.4.2. A unital product on an $H$-graded category $\mathbf{E}$ is a quadruple $(\bar{\otimes}, \mathcal{O}, \lambda, \rho)$ where:

(i) $\bar{\otimes}: \mathbf{E} \otimes_{H} \mathbf{E} \rightarrow \mathbf{E}$ is an $H$-graded functor,

(ii) $\mathcal{O}$ is an object in $\mathbf{E}$ (whence, by (i), there are $H$-graded endofunctors of $\mathbf{E}$ taking $A \in \mathbf{E}$ to $\mathcal{O} \bar{\otimes} A$ and to $A \bar{\otimes} \mathcal{O}$, respectively), and

(iii) $\lambda:(\mathcal{O} \bar{\otimes}-) \stackrel{\sim}{\longrightarrow} \mathrm{id}_{\mathbf{E}}$ and $\rho:(-\bar{\otimes} \mathcal{O}) \stackrel{\sim}{\longrightarrow} \mathrm{id}_{\mathbf{E}}$ are degree-0 functorial isomorphisms such that $\lambda_{\mathcal{O}}=\rho_{\mathcal{O}}: \mathcal{O} \bar{\otimes} \mathcal{O} \sim \mathcal{O}$.

1.4.3. When such a unital product is given, one verifies that the map that takes $\eta \in \mathbf{E}^{n}(\mathcal{O}, \mathcal{O})$ to the family $\left(\eta_{A}\right)_{A \in \mathbf{E}}$ in $\mathcal{C}^{n}$ such that $\eta_{A}$ is the composite map

$$
A \underset{\lambda_{A}^{-1}}{\stackrel{\sim}{\longrightarrow}} \mathcal{O} \bar{\otimes} A \underset{\eta \bar{\otimes} \operatorname{id}_{A}}{\longrightarrow} \mathcal{O} \bar{\otimes} A \underset{\lambda_{A}}{\stackrel{\sim}{\longrightarrow}} A
$$

is a homomorphism of graded $H$-algebras, right-inverse to ev $_{\mathcal{O}}: \mathcal{C} \rightarrow \mathbf{E}(\mathcal{O}, \mathcal{O})$ (see $(1.3 .1))$.

Thus $\mathbf{E}(\mathcal{O}, \mathcal{O})$ is a graded- $H$-algebra retract of $\mathcal{C}$, and so it is a gradedcommutative $H$-algebra; and the $\mathcal{C}$-grading on $\mathbf{E}$ induces an $\mathbf{E}(\mathcal{O}, \mathcal{O})$-grading.

2. The underlying setup. We now describe the formalism from which a bivariant theory will emerge in sections 3 and 4 . The formalism will be illustrated in section 5 by several instances involving Grothendieck duality.

2.1. Fix a category $\mathrm{S}$ and a graded-commutative ring $H$.

An orientation of a relation $f \circ v=u \circ g$ among four S-maps is an ordered pair (right arrow, bottom arrow) whose members are $f$ and $u$. This can be represented by one of two oriented commutative squares, namely $\mathbf{d}$ with bottom arrow $u$, and its transpose $\mathbf{d}^{\prime}$ with bottom arrow $f$.
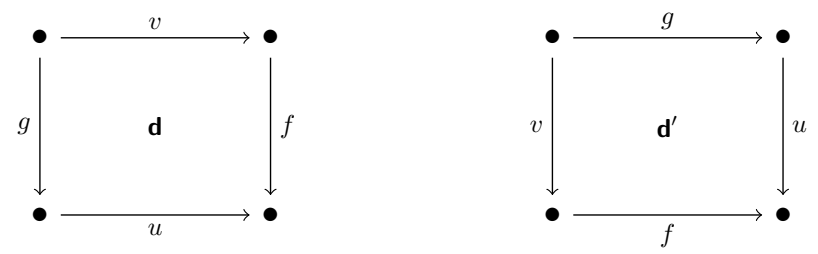

Assume that the category $\mathrm{S}$ is equipped with a class of maps, whose members are called confined maps, and a class of oriented commutative squares, whose members are called independent squares; and that these classes satisfy (A1), (A2), (B1), (B2) and $(\mathrm{C})$ in [FM, $§ 2.1]$ - identity maps and composites of confined maps are confined, vertical and horizontal composites of independent squares are independent, any $\mathbf{d}$ in which $f=g$ and in which $u$ and $v$ are identity maps is independent, and if in the independent square $\mathbf{d}$ the map $f$ (resp. $u$ ) is confined then so is $g$ (resp. $v$ ).

2.2. With terminology as in $\S 1$, assume given:

(i) for each object $W \in \mathrm{S}$ an $H$-graded category $\mathbf{D}_{W}$, and

(ii) contravariant $H$-graded pseudofunctors $(-)^{*}$ and $(-)^{\text {! }}$ over $\mathrm{S}$, with values in the categories $\mathbf{D}_{W}$-that is, to each $f: X \rightarrow Y$ in $\mathbf{S}$ there are assigned $H$-graded functors $f^{*}$ and $f^{!}$from $\mathbf{D}_{Y}$ to $\mathbf{D}_{X}$; and to each $X \stackrel{f}{\rightarrow} Y \stackrel{g}{\rightarrow} Z$ in $\mathbf{S}$ there are assigned 
functorial isomorphisms of degree 0

$$
\mathrm{ps}^{*}: f^{*} g^{*} \stackrel{\sim}{\longrightarrow}(g f)^{*}, \quad \mathrm{ps}^{!}: f^{!} g^{!} \stackrel{\sim}{\longrightarrow}(g f)^{!}
$$

such that for any $X \stackrel{f}{\rightarrow} Y \stackrel{g}{\rightarrow} Z \stackrel{h}{\rightarrow} W$ in S, the corresponding diagrams
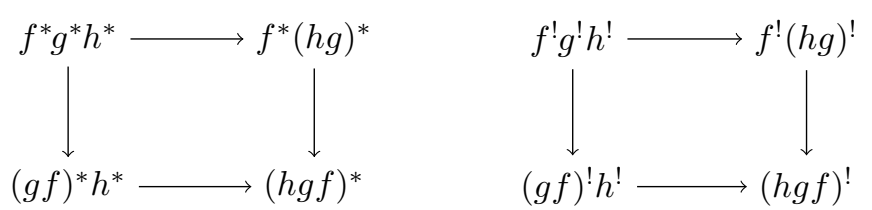

commute.

Replacing $(-)^{*}$ and $(-)^{!}$by isomorphic pseudofunctors, we may assume further that if $f$ is the identity map of $X$, then $f^{*}$ (resp. $f^{!}$) is the identity functor of $\mathbf{D}_{X}$, and that ps* (resp. ps!) is the identity transformation of the functor $g^{*}$ (resp. $g^{!}$); and likewise if $g$ is the identity map of $Y$.

Suggesting identification via $\mathrm{ps}^{*}$ or $\mathrm{ps}^{!}$, the notations

$$
f^{*} g^{*} \stackrel{\mathrm{ps}^{*}}{=}(g f)^{*}, \quad \quad f^{!} g^{!} \stackrel{\mathrm{ps} !}{=}(g f)^{!},
$$

will be used to represent these functorial isomorphisms or their inverses.

Henceforth, any pseudofunctor under consideration will be assumed to have been modified so as to exhibit the above-described simple behavior with respect to identity maps.

2.3. Assume that there is assigned to each independent square

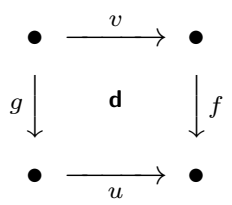

a degree-0 isomorphism of $H$-graded functors

$$
\mathrm{B}_{\mathbf{d}}: v^{*} f^{!} \stackrel{\sim}{\longrightarrow} g^{!} u^{*}
$$

These $B_{\mathbf{d}}$ are to satisfy horizontal and vertical transitivity: if the composite square $\mathbf{d}_{0}=\mathbf{d}_{2} \circ \mathbf{d}_{1}$ (with $g$ resp. $v$ deleted)
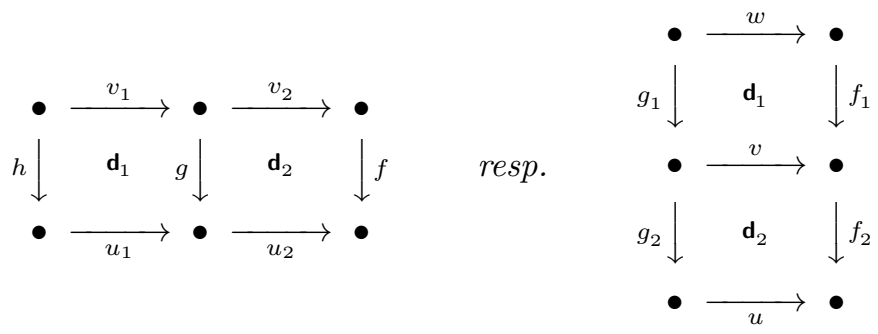
has independent constituents $\mathbf{d}_{2}$ and $\mathbf{d}_{1}$ (so that $\mathbf{d}_{0}$ itself is independent), then the corresponding natural diagram of functorial maps commutes:

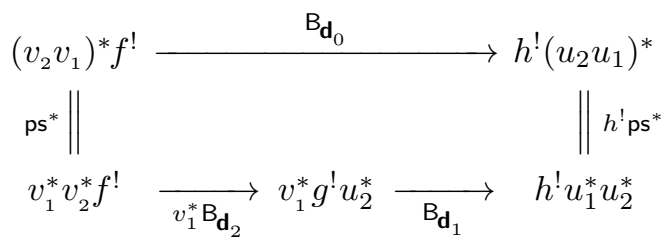

resp.

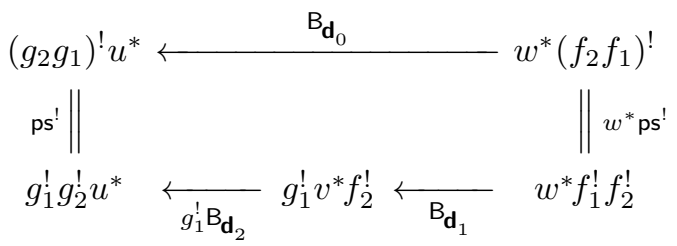

Assume further that if $u$ and $v$ are identity maps, or if $f$ and $g$ are identity maps, then $B_{\mathbf{d}}$ is the identity transformation.

2.4. Assume given a covariant H-graded pseudofunctor $(-)_{*}$ (that is, a contravariant $H$-graded pseudofunctor over the opposite category $S^{\text {op }}$ ), with values in the categories $\mathbf{D}_{W}$. Thus there are degree-0 functorial isomorphisms $\mathrm{ps}_{*}:(g f)_{*} \stackrel{\sim}{\longrightarrow} g_{*} f_{*}$ satisfying the appropriate analogs of (2.2.1) and the remarks after it. This isomorphism or its inverse will be represented as

$$
(g f)_{*} \stackrel{\mathrm{ps}_{*}}{=} g_{*} f_{*} .
$$

Assume further that this pseudofunctor is pseudofunctorially right-adjoint to $(-)^{*}$ : for any S-map $f: X \rightarrow Y$, the functor $f_{*}: \mathbf{D}_{X} \rightarrow \mathbf{D}_{Y}$ is graded right-adjoint to $f^{*}: \mathbf{D}_{Y} \rightarrow \mathbf{D}_{X}$, that is, there are degree-0 functorial unit and counit maps

$$
\eta=\eta_{f}: \mathrm{id} \rightarrow f_{*} f^{*} \quad \text { and } \quad \epsilon=\epsilon_{f}: f^{*} f_{*} \rightarrow \mathrm{id}
$$

such that for $A \in \mathbf{D}_{Y}$ and $C \in \mathbf{D}_{X}$ the corresponding compositions

$$
f_{*} A \stackrel{\eta_{f_{*} A}}{\longrightarrow} f_{*} f^{*} f_{*} A \stackrel{f_{*} \epsilon_{A}}{\longrightarrow} f_{*} A, \quad f^{*} C \stackrel{f^{*} \eta_{C}}{\longrightarrow} f^{*} f_{*} f^{*} C \stackrel{\epsilon_{f *} C}{\longrightarrow} f^{*} C
$$

are identity maps - or equivalently, the induced composite maps of symmetric graded $H$-modules

$$
\begin{aligned}
& \mathbf{D}_{Y}\left(A, f_{*} C\right) \rightarrow \mathbf{D}_{X}\left(f^{*} A, f^{*} f_{*} C\right) \rightarrow \mathbf{D}_{X}\left(f^{*} A, C\right), \\
& \mathbf{D}_{X}\left(f^{*} A, C\right) \rightarrow \mathbf{D}_{Y}\left(f_{*} f^{*} A, f_{*} C\right) \rightarrow \mathbf{D}_{Y}\left(A, f_{*} C\right)
\end{aligned}
$$

are inverse isomorphisms; and for any $X \stackrel{f}{\rightarrow} Y \stackrel{g}{\rightarrow} Z$ in $\mathrm{S}$, the following diagram commutes:

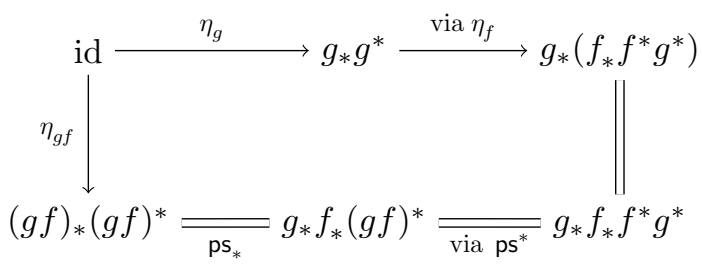


Assume also that to each confined map $f: X \rightarrow Y$ in $\mathrm{S}$ there is assigned a degree-0 functorial map

$$
\int_{f}: f_{*} f^{!} \rightarrow \mathrm{id}
$$

satisfying transitivity: for any $X \stackrel{f}{\rightarrow} Y \stackrel{g}{\rightarrow} Z$ in S with $f$ and $g$ confined, the following diagram commutes

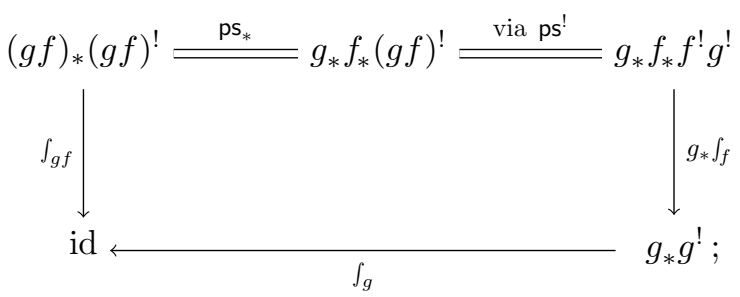

and if $f$ is the identity map of $X$ then $\int_{f}$ is the identity transformation.

2.5. Associated to any oriented commutative square in $S$

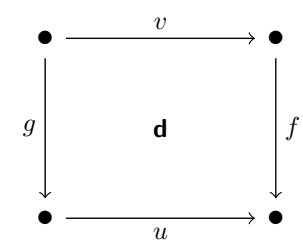

is the degree-0 functorial map

$$
\theta_{\mathbf{d}}: u^{*} f_{*} \rightarrow g_{*} v^{*}
$$

adjoint to

$$
f_{*} \stackrel{f_{*} \eta_{v}}{\longrightarrow} f_{*} v_{*} v^{*} \stackrel{\mathrm{ps}_{*}}{=} u_{*} g_{*} v^{*},
$$

i.e., $\theta_{\mathbf{d}}$ is the composition of the following chain of functorial maps:

$$
u^{*} f_{*} \stackrel{\text { via } \eta_{v}}{\longrightarrow} u^{*} f_{*} v_{*} v^{*} \stackrel{\text { via ps }}{=} u^{*} u_{*} g_{*} v^{*} \stackrel{\epsilon_{u}}{\longrightarrow} g_{*} v^{*} .
$$

It is postulated that if $\mathbf{d}$ is independent then $\theta_{\mathbf{d}}$ is an isomorphism.

2.6. Finally, it is postulated that if $\mathbf{d}$ in 2.5 is independent and $f$ (hence $g$ ) is confined, then the following diagram commutes

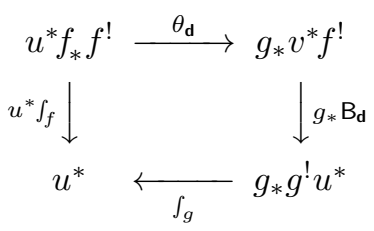


that is, the following diagram commutes

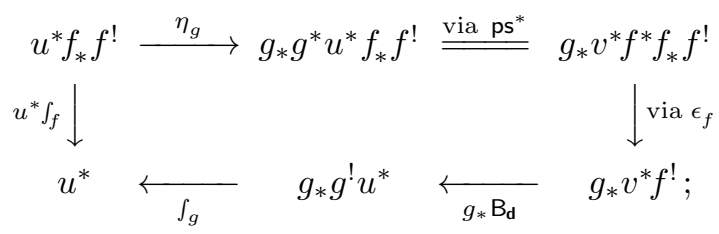

and if, in addition, $u$ (hence $v$ ) is confined, then with $\phi_{\mathbf{d}}$ the degree-0 functorial map adjoint to the composite map

$$
v^{*} f^{!} u_{*} \stackrel{\mathrm{B}_{\mathbf{d}}}{\longrightarrow} g^{!} u^{*} u_{*} \stackrel{g^{!} \epsilon_{u}}{\longrightarrow} g^{!},
$$

the following diagram commutes

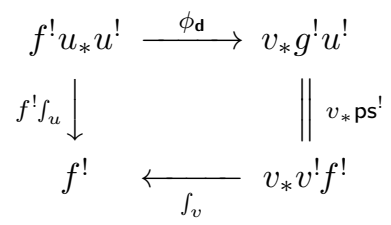

that is, the following diagram commutes

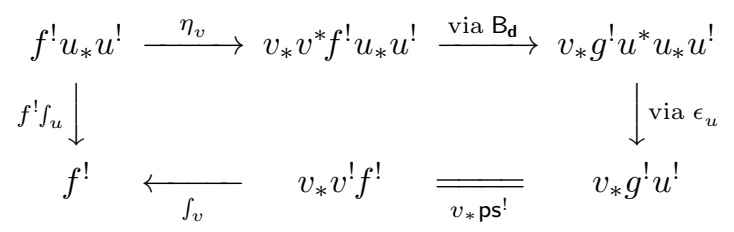

This completes the description of the underlying setup.

REMARK. The order of composition of the functors in the domain and target of $\theta_{\mathbf{d}}: u^{*} f_{*} \rightarrow g_{*} v^{*}$ indicates that we are considering that orientation of the relation $f \circ v=u \circ g$ for which $u$ is the bottom arrow. So when such a relation is given, we usually simplify notation by writing $\theta: u^{*} f_{*} \rightarrow g_{*} v^{*}$ instead of $\theta_{\mathbf{d}}: u^{*} f_{*} \rightarrow g_{*} v^{*}$; and likewise for $B_{\mathbf{d}}$ and $\phi_{\mathbf{d}}$.

\section{Defining a bivariant theory.}

3.1. In this section, we define data that will be shown in the next section to constitute a bivariant theory $[\mathrm{FM}]$. The approach will be purely formal, but justified by concrete examples (see 3.5 and $\S 6$ ).

3.1.1. Fix a setup, that is, a category $S$ with confined maps and independent squares, a graded-commutative ring $H$, a family $\left(\mathbf{D}_{W}\right)_{W \in \mathrm{S}}$ of $H$-graded categories, $H$-graded $\mathbf{D}_{W}$-valued pseudofunctors $(-)^{*},(-)^{!}$and $(-)_{*}$ over $\mathbf{S}$ (the first two contravariant and the last covariant), for each independent square $\mathbf{d}$, degree-0 functorial isomorphisms $\mathbf{B}_{\mathbf{d}}$ and $\theta_{\mathbf{d}}$, for each S-map $f$, degree-0 functorial maps

$$
\eta=\eta_{f}: \mathrm{id} \rightarrow f_{*} f^{*} \quad \text { and } \quad \epsilon=\epsilon_{f}: f^{*} f_{*} \rightarrow \mathrm{id},
$$

and for each confined map, a degree-0 functorial map

$$
\int_{f}: f_{*} f^{!} \rightarrow \mathrm{id}
$$

all subject to the conditions specified in $\S 2$. Assume also that $\mathrm{S}$ has a final object $S$. 
3.1.2. One associates to the pseudofunctor $(-)^{*}$ the "fibered category" $p: F \rightarrow S$, where the category $\mathrm{F}$ has as objects the pairs $(W, C)$ such that $W \in \mathrm{S}$ and $C \in \mathbf{D}_{W}$, and as morphisms the pairs $(f, \psi):(X, A) \rightarrow(Y, B)$ such that $f: X \rightarrow Y$ is an S-map and $\psi: f^{*} B \rightarrow A$ is a $\mathbf{D}_{X}$-map, the composition of such morphisms being defined in the obvious way, and where the functor $\mathrm{p}$ is "projection to the first coordinate." The bivariant theory will be constructed from a section s - a right inverse - of p. Such an $\mathrm{s}$ can be specified without reference to $\mathrm{F}$ or $\mathrm{p}$, see $\S 3.2$.

For any $W \in \mathrm{S}$, set $\left(W, \mathcal{H}_{W}\right):=\mathrm{s}(W)$. (This notation reflects our original motivation, the case where $\mathcal{H}_{X}$ is a Hochschild complex, see example 3.5(b) below.)

Assume throughout that if $f: X \rightarrow Y$ is the bottom or top arrow of an independent square, then the s-induced map $f^{*} \mathcal{H}_{Y} \rightarrow \mathcal{H}_{X}$ is an isomorphism.

We say that an S-map is co-confined if it is represented by the bottom arrow of some independent square.

To each S-map $f: X \rightarrow Y$ is attached the symmetric graded $H$-module

$$
\mathrm{HH}^{*}(X \stackrel{f}{\rightarrow} Y):=\mathbf{D}_{X}\left(\mathcal{H}_{X}, f^{!} \mathcal{H}_{Y}\right)=\oplus_{i \in \mathbb{Z}} \mathbf{D}_{X}^{i}\left(\mathcal{H}_{X}, f^{!} \mathcal{H}_{Y}\right)
$$

We will define graded homomorphisms between such modules-products, pushforwards via confined maps, and pullbacks via independent squares - and then verify in the next section that for these operations in the given setup, the axioms of a bivariant theory hold.

3.1.3. There result homology groups, covariant for confined S-maps,

$$
\mathrm{HH}_{i}(X):=\mathbf{D}_{X}^{-i}\left(\mathcal{H}_{X}, x^{!} \mathcal{H}_{S}\right) \quad(i \in \mathbb{Z})
$$

where $x: X \rightarrow S$ is the unique S-map; and cohomology groups, contravariant for co-confined S-maps,

$$
\operatorname{HH}^{i}(X):=\mathbf{D}_{X}^{i}\left(\mathcal{H}_{X}, \mathcal{H}_{X}\right),
$$

see $[\mathrm{FM}, \S 2.3]$. As in $\S 1.1 .1$,

$$
\mathrm{HH}^{*}(X):=\oplus_{i \in \mathbb{Z}} \mathrm{HH}^{i}(X)=\mathbf{D}_{X}\left(\mathcal{H}_{X}, \mathcal{H}_{X}\right)
$$

is a graded $H$-algebra. (We will actually focus on the opposite $H$-algebra.) Composition of $\mathbf{D}_{X}$-maps makes the symmetric graded $H$-module

$$
\mathrm{HH}_{*}(X):=\oplus_{i \in \mathbb{Z}} \mathrm{HH}_{-i}(X)=\mathbf{D}_{X}\left(\mathcal{H}_{X}, x^{!} \mathcal{H}_{S}\right)
$$

into a graded right $\mathrm{HH}^{*}(X)$-module (= graded left module over the opposite algebra).

By way of illustration, we will indicate in $\S 6$ the relation to the present formalism of some previously defined Hochschild homology and cohomology functors on schemes.

3.2. We now begin the detailed description of a bivariant theory.

Fix a setup $(\mathrm{S}, H, \ldots)$ as in 3.1.1. Our construction assumes given:

(i) For each $X \in \mathrm{S}$ an object $\mathcal{H}_{X} \in \mathbf{D}_{X}$.

(ii) For each S-map $f: X \rightarrow Y$ a $\mathbf{D}_{X}$-morphism

$$
f^{\sharp}: f^{*} \mathcal{H}_{Y} \rightarrow \mathcal{H}_{X},
$$


such that

(iii) if $f$ is an identity map then so is $f^{\sharp}$, and

(iv) (transitivity) for S-maps $X \stackrel{f}{\rightarrow} Y \stackrel{g}{\rightarrow} Z$ the next diagram commutes:

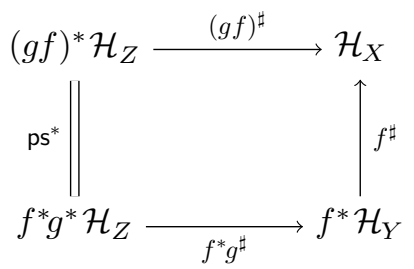

It is further assumed that if $f: X \rightarrow Y$ is the bottom or top arrow of an independent square, then $f^{\sharp}$ is an isomorphism.

The adjoint of the map $f^{\sharp}$ will be denoted $f_{\sharp}: \mathcal{H}_{Y} \rightarrow f_{*} \mathcal{H}_{X}$.

LEMMa 3.2.2.

Let $X \stackrel{f}{\rightarrow} Y \stackrel{g}{\rightarrow} Z$ be S-maps. The next diagram commutes.

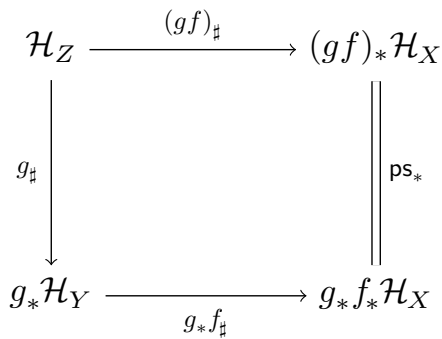

Proof. The diagram expands as follows:

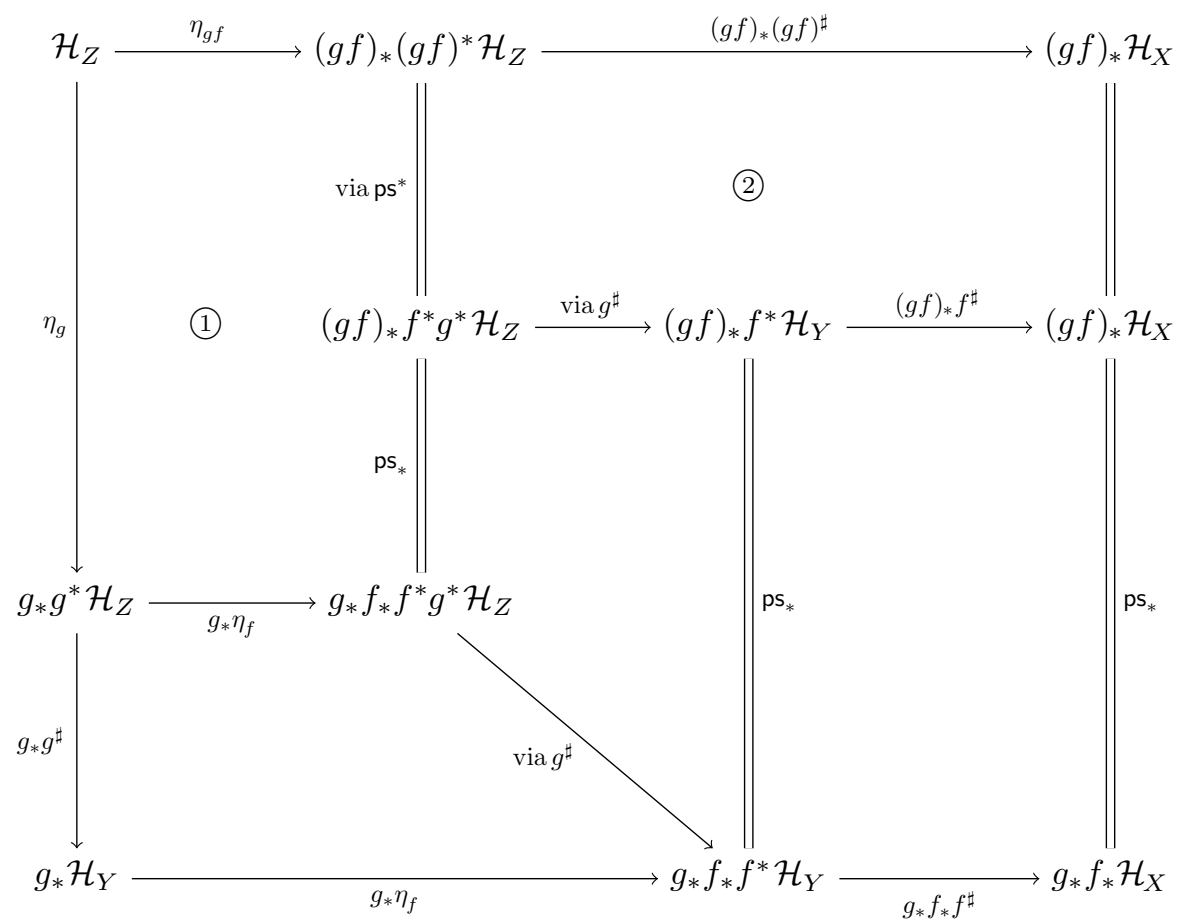


Commutativity of subdiagram (1) is given by (2.4.2); of (2) by (3.2.1); and of the remaining subdiagrams is obvious.

3.3. Associate to any S-map $f: X \rightarrow Y$ the symmetric graded $H$-module

$$
\mathrm{HH}^{*}(X \stackrel{f}{\rightarrow} Y):=\mathbf{D}_{X}\left(\mathcal{H}_{X}, f^{!} \mathcal{H}_{Y}\right)=\oplus_{i \in \mathbb{Z}} \mathbf{D}_{X}^{i}\left(\mathcal{H}_{X}, f^{!} \mathcal{H}_{Y}\right) .
$$

There are three basic bivariant operations on these $H$-modules, as follows.

3.3.2. Product. Let $f: X \rightarrow Y$ and $g: Y \rightarrow Z$ be maps in S.

For $i, j \in \mathbb{Z}$ and $\alpha \in \mathrm{HH}^{i}(X \stackrel{f}{\rightarrow} Y), \beta \in \mathrm{HH}^{j}(Y \stackrel{g}{\rightarrow} Z)$, let the product

$$
\alpha \cdot \beta \in \mathrm{HH}^{i+j}(X \stackrel{g f}{\longrightarrow} Z)
$$

be $(-1)^{i j}$ times the composite map

$$
\mathcal{H}_{X} \stackrel{\alpha}{\longrightarrow} f^{!} \mathcal{H}_{Y} \stackrel{f^{!} \beta}{\longrightarrow} f^{!} g^{!} \mathcal{H}_{Z} \stackrel{\mathrm{ps}}{=}(g f)^{!} \mathcal{H}_{Z}
$$

Since composition $\mathrm{S}$ is $H$-bilinear, since $f^{!}$is a graded functor and since $\mathrm{ps}^{!}\left(\mathcal{H}_{Z}\right)$ has degree 0 , therefore this product gives a graded $H$-bilinear map

$$
\mathrm{HH}^{*}(X \stackrel{f}{\longrightarrow} Y) \times \mathrm{HH}^{*}(Y \stackrel{g}{\longrightarrow} Z) \longrightarrow \mathrm{HH}^{*}(X \stackrel{g f}{\longrightarrow} Z) .
$$

For the case when $X=Y$ and $f=$ identity, the identity map of $\mathcal{H}_{X}$ is a left unit for the product. Similarly when $Y=Z$ and $g=$ identity, the identity map of $\mathcal{H}_{Z}$ is a right unit.

3.3.3. Pushforward. Let $f: X \rightarrow Y$ and $g: Y \rightarrow Z$ be maps in S, with $f$ confined. The pushforward by $f$

$$
f_{\star}: \mathrm{HH}^{*}(X \stackrel{g f}{\rightarrow} Z) \rightarrow \mathrm{HH}^{*}(Y \stackrel{g}{\rightarrow} Z)
$$

is the graded $H$-linear map such that for $i \in \mathbb{Z}$ and $\alpha \in \mathrm{HH}^{i}(X \stackrel{g f}{\longrightarrow} Z)$, the image $f_{\star} \alpha \in \mathrm{HH}^{i}(Y \stackrel{g}{\rightarrow} Z)$ is the natural composition

$$
\mathcal{H}_{Y} \stackrel{f_{\sharp}}{\longrightarrow} f_{*} \mathcal{H}_{X} \stackrel{f_{*} \alpha}{\longrightarrow} f_{*}(g f) ! \mathcal{H}_{Z} \stackrel{f_{*} \mathrm{ps} !}{=} f_{*} f^{!} g^{!} \mathcal{H}_{Z} \stackrel{\int_{f}}{\longrightarrow} g^{!} \mathcal{H}_{Z} .
$$

In other words, $f_{\star} \alpha$ is the composition

$$
\mathcal{H}_{Y} \stackrel{f_{\sharp}}{\longrightarrow} f_{*} \mathcal{H}_{X} \stackrel{\widetilde{\alpha}}{\longrightarrow} g ! \mathcal{H}_{Z}
$$

where $\widetilde{\alpha}: f_{*} \mathcal{H}_{X} \rightarrow g^{!} \mathcal{H}_{Z}$ is the map obtained by adjunction from

$$
\mathcal{H}_{X} \stackrel{\alpha}{\longrightarrow}(g f)^{!} \mathcal{H}_{Z} \stackrel{\mathrm{ps} !}{=} f^{!} g^{!} \mathcal{H}_{Z}
$$


3.3.4. Pullback. Let $\mathbf{d}$ be an independent square in $\mathrm{S}$

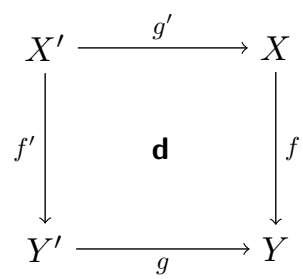

The maps $g^{\sharp}: g^{*} \mathcal{H}_{Y} \rightarrow \mathcal{H}_{Y^{\prime}}$ and $g^{\prime \sharp}: g^{\prime *} \mathcal{H}_{X} \rightarrow \mathcal{H}_{X^{\prime}}$ are isomorphisms (§3.2).

The pullback by $g$, through $\mathbf{d}$,

$$
g^{\star}: \mathrm{HH}^{*}(X \stackrel{f}{\rightarrow} Y) \longrightarrow \mathrm{HH}^{*}\left(X^{\prime} \stackrel{f^{\prime}}{\rightarrow} Y^{\prime}\right)
$$

is the graded $H$-linear map such that for $i \in \mathbb{Z}$ and $\alpha \in \mathrm{HH}^{i}(X \stackrel{f}{\rightarrow} Y)$, the image $g^{\star} \alpha \in \mathrm{HH}^{i}\left(X^{\prime} \stackrel{f^{\prime}}{\longrightarrow} Y^{\prime}\right)$ is the natural composition

$$
\mathcal{H}_{X^{\prime}} \stackrel{\left(g^{\prime \sharp}\right)^{-1}}{\longrightarrow} g^{\prime *} \mathcal{H}_{X} \stackrel{g^{\prime *} \alpha}{\longrightarrow} g^{\prime *} f^{!} \mathcal{H}_{Y} \stackrel{\mathrm{B}_{\mathbf{d}}}{\longrightarrow} f^{\prime !} g^{*} \mathcal{H}_{Y} \stackrel{f^{\prime !}\left(g^{\sharp}\right)}{\longrightarrow} f^{\prime !} \mathcal{H}_{Y^{\prime}} .
$$

For $X=Y, X^{\prime}=Y^{\prime}, f$ and $f^{\prime}$ identity maps, pullback takes the identity map of $\mathcal{H}_{X}$ to that of $\mathcal{H}_{X^{\prime}}$

Thus identity maps are units in the sense of [FM, p.22].

ThEOREM 3.4. The data in sections 3.2-3.3 constitute a bivariant theory, with units, on $\mathrm{S}$, taking values in symmetric graded $H$-modules.

The proof of Theorem 3.4-that is, the verification of the bivariance axioms - is given in $\S 4$.

In the rest of this section, we discuss some examples, and their associated bivariant homology-cohomology pairs.

EXAMPLES 3.5. In $\S 5$ we will show in detail that there is a setup in which $\mathrm{S}$ is a category of essentially-finite-type perfect (i.e., finite tor-dimension) separated maps of noetherian schemes, closed under fiber product and having a final object $S$, with proper maps as confined maps, and oriented fiber squares with flat bottom arrow as independent squares; and in which $H:=\oplus_{i \geq 0} H^{i}\left(S, \mathcal{O}_{S}\right)$ with its natural commutative-graded ring structure. Moreover, for each $X \in \mathbf{S}, \mathbf{D}_{X}$ is the full subcategory $\mathbf{D}_{\mathrm{qc}}(X)$ of the derived category $\mathbf{D}(X)$ - enriched in the standard way with an $\mathrm{H}$ graded structure - such that an $\mathcal{O}_{X}$-complex $C$ is an object of $\mathbf{D}_{\mathrm{qc}}(X)$ if and only if all the homology sheaves of $C$ are quasi-coherent; and for any $S$-map $f: X \rightarrow Y, f^{*}$ is the graded enrichment of the derived inverse-image functor (usually denoted $L f^{*}$ ).

The following examples refer to such a setup.

(a) Fix an object $\mathcal{H}_{S} \in \mathbf{D}_{S}$. For each $X \in \mathrm{S}$, with its unique S-map $x: X \rightarrow S$, set $\mathcal{H}_{X}:=x^{*} \mathcal{H}_{S}$. For an S-map $f$, let $f^{\sharp}$ be $f^{*} x^{*} \mathcal{H}_{S} \stackrel{\text { ps }}{=}(x f)^{*} \mathcal{H}_{S}$.

(b) For each $X \in \mathrm{S}$ let $\mathcal{H}_{X}$ be the Hochschild complex $\mathbb{H}_{X / S}$, and $f^{\sharp}$ as explained in the proof of [BF1, Theorem 1.3].

(c) For each $X \in \mathrm{S}$, let $\mathcal{H}_{X}$ be the cotangent complex $L_{X / S}$, and $f^{\sharp}$ the map given by [Il, p. 132, (1.2.7.2)] (with $Y=Y^{\prime}:=S$ ). 
Examples (b) and (c) are not unrelated — see [BF2, Theorem 3.1.3].

(d) There are many ways to get new families satisfying 3.2(i)-(iv) from old ones. For example, to two such families $\left(\mathcal{H}_{X, 1}, f_{1}^{\sharp}\right)$ and $\left(\mathcal{H}_{X, 2}, f_{2}^{\sharp}\right)$, apply the derived tensor product functor, or the direct sum functor, or ...

3.5.1. In examples 3.5(b) and (c), if an S-map $f: X \rightarrow Y$ is essentially étale (see $\S 5.7$ below) then $f^{*} \mathcal{H}_{Y} \rightarrow \mathcal{H}_{X}$ is an isomorphism. (The assertion for Example (b) will be treated in a sequel to this paper. Example (c) is covered by [Il, p. 135, 2.1.2.1 and p. 203, 3.1.1].) So for these examples, one needs, according to $§ 3.2$, to restrict the class of independent squares to those fiber squares whose bottom (hence top) arrow is essentially étale.

No such restriction is needed in Example (a).

3.6. The bivariant theory provides symmetric graded $H$-modules

$$
\mathrm{HH}^{*}(X):=\mathrm{HH}^{*}(X \stackrel{\mathrm{id}}{\rightarrow} X)=\mathbf{D}_{X}\left(\mathcal{H}_{X}, \mathcal{H}_{X}\right)=\oplus_{i \in \mathbb{Z}} \mathbf{D}_{X}^{i}\left(\mathcal{H}_{X}, \mathcal{H}_{X}\right)
$$

(bivariant cohomology), and, with $x: X \rightarrow S$ the unique S-map,

$$
\mathrm{HH}_{*}(X):=\mathrm{HH}^{*}(X \stackrel{x}{\rightarrow} S)=\mathbf{D}_{X}\left(\mathcal{H}_{X}, x^{!} \mathcal{H}_{S}\right)=\oplus_{i \in \mathbb{Z}} \mathbf{D}_{X}^{-i}\left(\mathcal{H}_{X}, x^{!} \mathcal{H}_{S}\right)
$$

(bivariant homology).

For instance, if, in $3.5(\mathrm{a}), \mathcal{H}_{S}=\mathcal{O}_{S}$, then bivariant cohomology is just

$$
\mathrm{HH}^{i}(X)=\mathrm{H}^{i}\left(X, \mathcal{O}_{X}\right)
$$

and homology is the (hyper)cohomology of the relative dualizing complex:

$$
\mathrm{HH}_{i}(X)=\mathrm{H}^{-i}\left(X, x^{!} \mathcal{O}_{S}\right) .
$$

For the bivariant Hochschild theory of example 3.5(b), the corresponding (co)homology is discussed - at least for flat maps - in $\S 6$.

Functoriality, basic properties of, and operations between, $\mathrm{HH}^{*}$ and $\mathrm{HH}_{*}$ result from the structure of a bivariant theory, and correspond to the usual structure of a theory of cohomology and homology, as follows.

The cup product

$$
\smile: \mathrm{HH}^{i}(X) \otimes \mathrm{HH}^{j}(X) \longrightarrow \mathrm{HH}^{i+j}(X)
$$

is the product 3.3.2 associated to the composition $X \stackrel{\text { id }}{\rightarrow} X \stackrel{\text { id }}{\rightarrow} X$ : for each $\alpha \in \mathbf{D}_{X}^{i}\left(\mathcal{H}_{X}, \mathcal{H}_{X}\right)$ and $\beta \in \mathbf{D}_{X}^{j}\left(\mathcal{H}_{X}, \mathcal{H}_{X}\right)$,

$$
\alpha \smile \beta:=(-1)^{i j} \beta \circ \alpha \in \mathbf{D}_{X}^{i+j}\left(\mathcal{H}_{X}, \mathcal{H}_{X}\right) .
$$

Cup product makes $\mathrm{HH}^{*}(X)$ into a graded ring-opposite to $\mathbf{D}_{X}\left(\mathcal{H}_{X}, \mathcal{H}_{X}\right)$ with its composition product. Both rings have the same graded center, and so $\operatorname{HH}^{*}(X)$ is a graded $H$-algebra.

As in $\S 1.3$, both $\mathrm{HH}^{*}(X)$ and $\mathrm{HH}_{*}(X)$ are actually symmetric graded modules over the graded center $\mathcal{C}_{X}$ of $\mathbf{D}_{X}$. In fact, since $\mathcal{C}_{X}$ is graded-commutative, the evaluation map (1.3.1) with $A=\mathcal{H}_{X}$ sends $\mathcal{C}_{X}$ to the graded center of $\operatorname{HH}^{*}(X)$, so that $\operatorname{HH}^{*}(X)$ is a graded $\mathcal{C}_{X}$-algebra. 
Recall that an S-map is co-confined if it is represented by the bottom arrow of some independent square.

It results from Proposition 4.5 below (with $X=Y=Z$ and $f=g=1$ ) that for every co-confined map $f: X^{\prime} \rightarrow X$, the graded $H$-linear pullback

$$
f^{\star}: \mathrm{HH}^{*}(X) \longrightarrow \mathrm{HH}^{*}\left(X^{\prime}\right)
$$

of 3.3.4 is a ring homomorphism.

Thus:

Proposition 3.6.1. With $\mathrm{S}_{\mathrm{co}}$ the subcategory of all co-confined maps in $\mathrm{S}$, bivariant cohomology with the cup product gives a contravariant functor

$$
\mathrm{HH}^{*}: \mathrm{S}_{\mathrm{co}} \rightarrow\{H \text {-algebras }\}
$$

For $x: X \rightarrow S$ in $\mathrm{S}$, the cap product

$$
\frown: \mathrm{HH}^{i}(X) \otimes \mathrm{HH}_{j}(X) \longrightarrow \mathrm{HH}_{j-i}(X)
$$

is defined to be the product associated to the composition $X \stackrel{\mathrm{id}}{\rightarrow} X \stackrel{x}{\rightarrow} S$ : for each $\alpha \in \mathbf{D}_{X}^{i}\left(\mathcal{H}_{X}, \mathcal{H}_{X}\right)$ and $\beta \in \mathbf{D}_{X}^{-j}\left(\mathcal{H}_{X}, x^{!} \mathcal{O}_{S}\right)$,

$$
\alpha \frown \beta:=(-1)^{i j} \beta \circ \alpha \in \mathbf{D}_{X}^{i-j}\left(\mathcal{H}_{X}, x^{!} \mathcal{O}_{S}\right) .
$$

With this product, $\mathrm{HH}_{*}(X)$ is a graded left $\mathrm{HH}^{*}(X)$-module.

Associated to a confined S-map $f: X^{\prime} \rightarrow X$ one has the $H$-linear pushforward of 3.3.3:

$$
f_{\star}: \mathrm{HH}_{*}\left(X^{\prime}\right) \longrightarrow \mathrm{HH}_{*}(X) .
$$

Thus:

Proposition 3.6.2. With $\mathrm{S}_{\mathrm{cf}}$ the subcategory of all confined maps in $\mathrm{S}$, bivariant homology together with the cap product, gives a covariant functor

$$
\left.\mathrm{HH}_{*}: \mathrm{S}_{\mathrm{cf}} \longrightarrow \text { \{symmetric graded } H \text {-modules }\right\} \text {. }
$$

Moreover, for every $X \in \mathrm{S}, \mathrm{HH}_{*}(X)$ is a graded left $\mathrm{HH}^{*}(X)$-module.

Proposition 4.7 (with $Z=S, f=\mathrm{id}_{X}, f^{\prime}=\mathrm{id}_{X^{\prime}}$ ) yields:

Proposition 3.6.3. If $g: X^{\prime} \rightarrow X$ is both confined and co-confined, then for all $\alpha \in \mathrm{HH}^{*}(X)$ and $\beta \in \mathrm{HH}_{*}\left(X^{\prime}\right)$,

$$
g_{\star}\left(g^{\star} \alpha \frown \beta\right)=\alpha \frown g_{\star} \beta .
$$

4. Checking the axioms. In this section we prove Theorem 3.4 by verifying that the axioms for a bivariant theory do hold for the data referred to in that theorem.

In the diagrams which appear, labels on the arrows are meant to indicate where the represented maps come from-usually by application of some obvious functors. Moreover, to reduce clutter we have hidden symbols like $[i]$ that denote translation functors; but the serious reader will easily find them. 
Recall from (3.3.1) that for an S-map $f: X \rightarrow Y$,

$$
\mathrm{HH}^{i}(X \stackrel{f}{\rightarrow} Y):=\mathbf{D}_{X}^{i}\left(\mathcal{H}_{X}, f^{!} \mathcal{H}_{Y}\right) \quad(i \in \mathbb{Z})
$$

Following [FM], we indicate that $\alpha \in \mathrm{HH}^{*}(X \stackrel{f}{\rightarrow} Y):=\oplus_{i \in \mathbb{Z}} \mathrm{HH}^{i}(X \stackrel{f}{\rightarrow} Y)$ by the notation

$$
X \underset{\propto}{\stackrel{f}{\propto}} Y
$$

Proposition 4.1. $\left(A_{1}\right)$ Associativity of product:

For any S-diagram

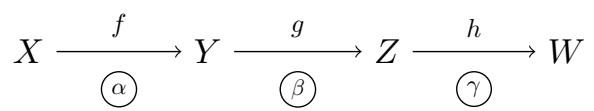

one has, in $\mathrm{HH}^{*}(X \stackrel{h g f}{\longrightarrow} W)$,

$$
(\alpha \cdot \beta) \cdot \gamma=\alpha \cdot(\beta \cdot \gamma)
$$

Proof. This property results from the obvious commutativity of the following diagram, with $\alpha \in \mathrm{HH}^{i}(X \stackrel{f}{\rightarrow} Y), \beta \in \mathrm{HH}^{j}(Y \stackrel{g}{\rightarrow} Z)$ and $\gamma \in \mathrm{HH}^{k}(Z \stackrel{h}{\rightarrow} W)$ :

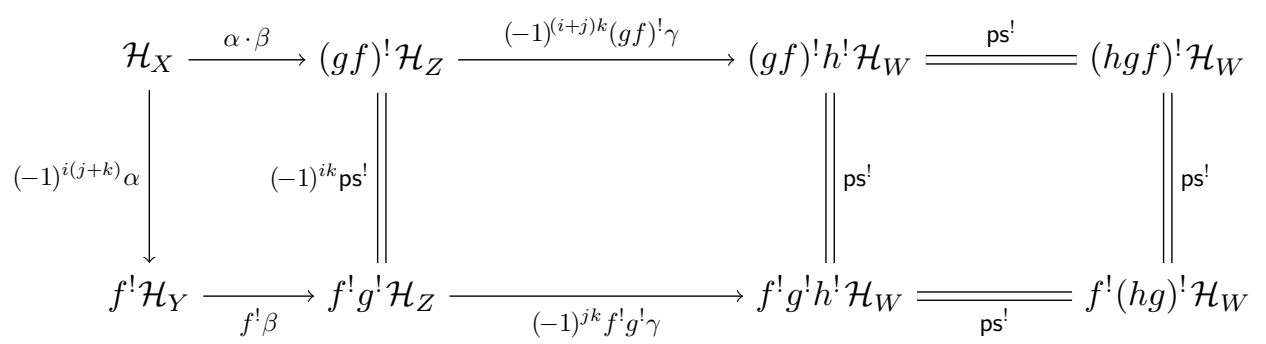

Proposition 4.2. $\left(A_{2}\right)$ Functoriality of pushforward:

For S-maps $f: X \rightarrow Y, g: Y \rightarrow Z$ and $h: Z \rightarrow W$, with $f$ and $g$ confined, and $\alpha \in \mathrm{HH}^{*}(X \stackrel{h g f}{\longrightarrow} W)$, one has, in $\mathrm{HH}^{*}(Z \stackrel{h}{\rightarrow} W)$,

$$
(g f)_{\star}(\alpha)=g_{\star} f_{\star}(\alpha) .
$$

Proof. We may assume, $\alpha \in \mathrm{HH}^{i}(X \stackrel{h g f}{\longrightarrow} W)$. What is then asserted is commutativity of the border of the following diagram: 


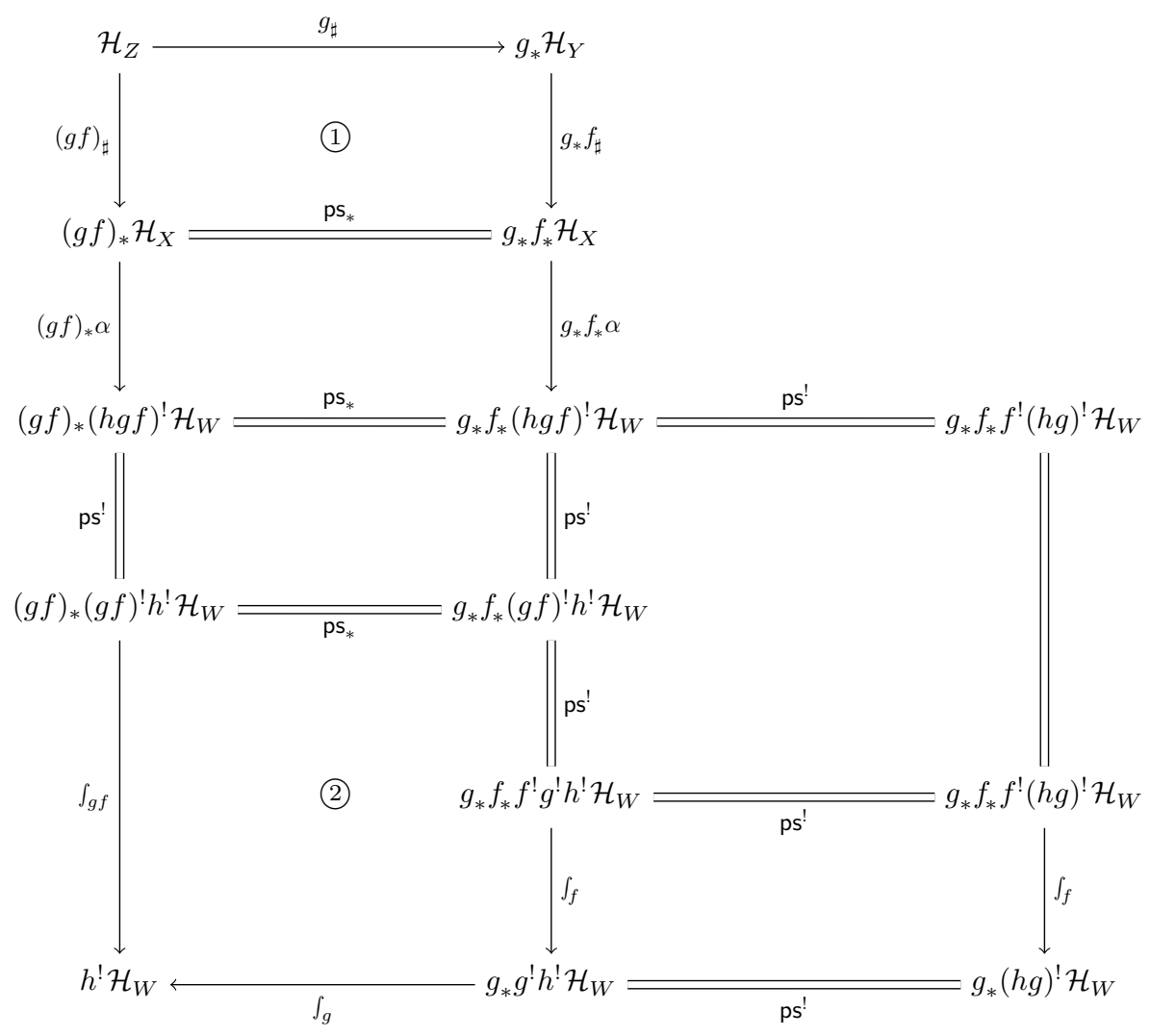

Commutativity of subdiagram (1) is given by Lemma 3.2.2. Commutativity of (2) (without $h^{!} \mathcal{H}_{W}$ ) results from that of (2.4.4). Commutativity of the unlabeled subdiagrams is clear. The result follows.

Proposition 4.3. $\left(A_{3}\right)$ Functoriality of pullback:

For any S-diagram, with independent squares,

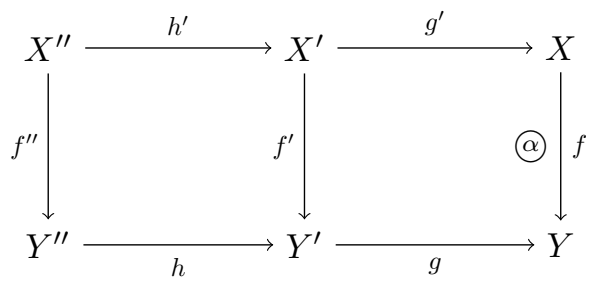

one has, in $\mathrm{HH}^{*}\left(X^{\prime \prime} \stackrel{f^{\prime \prime}}{\longrightarrow} Y^{\prime \prime}\right)$,

$$
(g h)^{\star}(\alpha)=h^{\star} g^{\star}(\alpha)
$$


Proof. The assertion amounts to commutativity of the border of the next diagram:

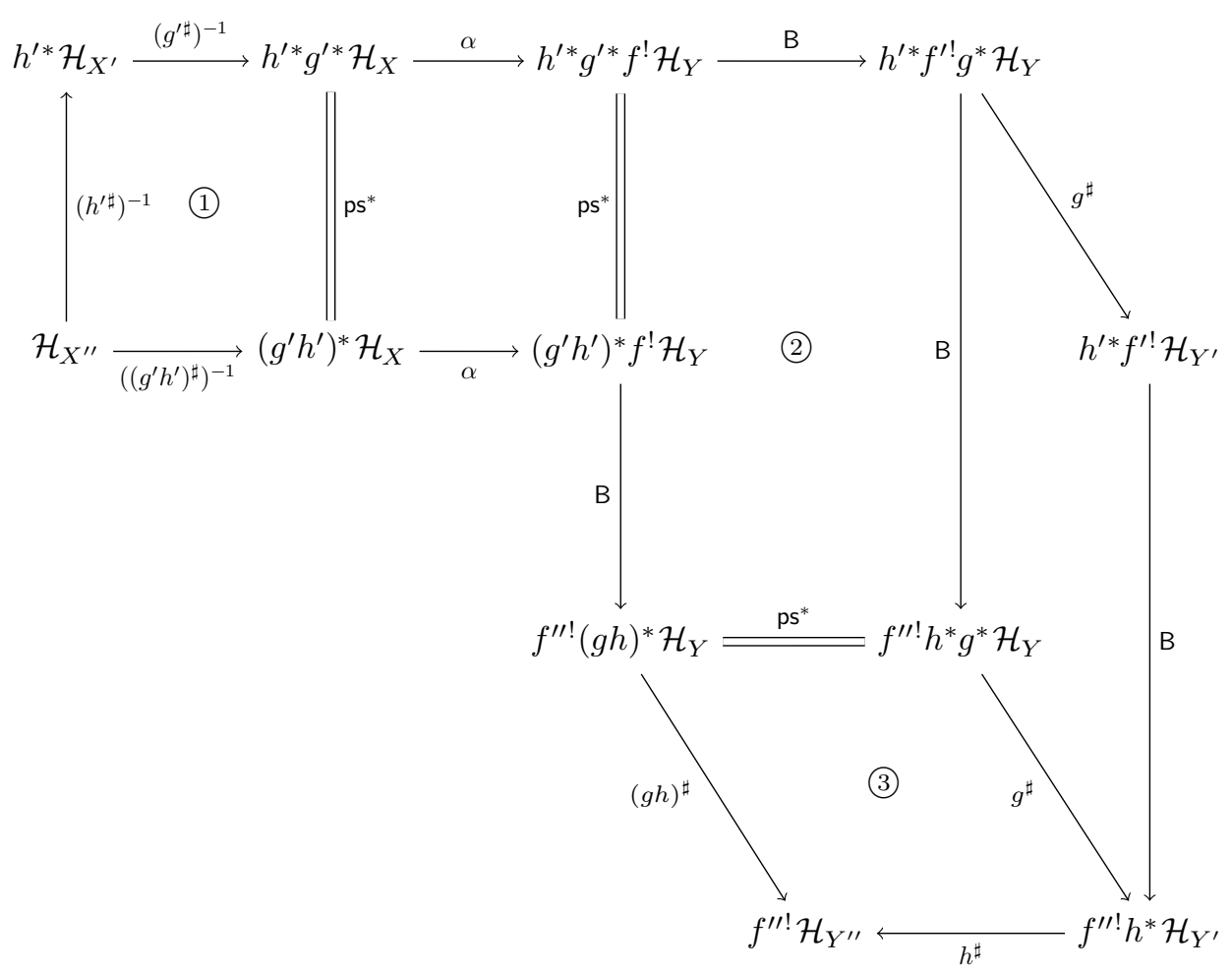

Subdiagrams (1) and (3) commute by 3.2(iv); subdiagram (2) commutes by (2.3.1); and commutativity of the other two subdiagrams is clear. The desired conclusion results. $\mathrm{C}$

Proposition 4.4. $\left(A_{12}\right)$ Product and pushforward commute:

For any S-diagram

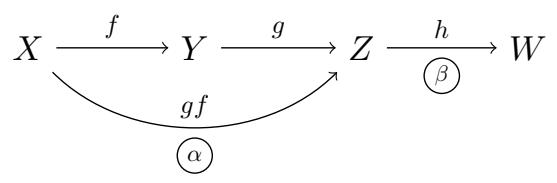

with $f: X \rightarrow Y$ confined, one has, in $\mathrm{HH}^{*}(Y \stackrel{h g}{\longrightarrow} W)$,

$$
f_{\star}(\alpha \cdot \beta)=f_{\star}(\alpha) \cdot \beta
$$

Proof. We may assume that $\alpha \in \mathrm{HH}^{i}(X \stackrel{g f}{\longrightarrow} Z)$ and $\beta \in \mathrm{HH}^{j}(Z \stackrel{h}{\rightarrow} W)$. Then 
what is asserted is commutativity of the border of the next diagram:

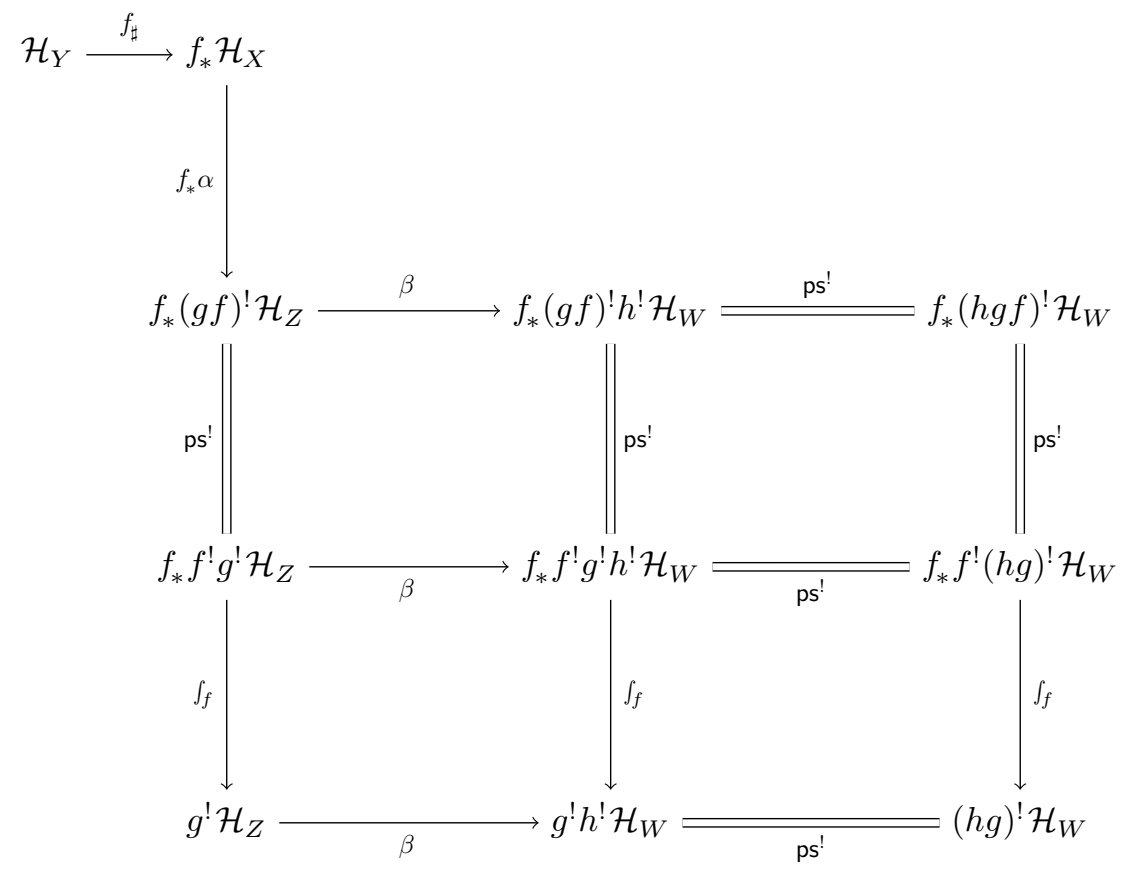

The subdiagrams obviously commute, whence the assertion.

Proposition 4.5. $\left(A_{13}\right)$ Product and pullback commute:

For any S-diagram with independent squares,

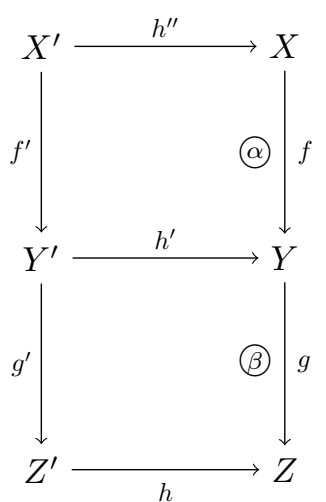

one has, in $\mathrm{HH}^{*}\left(X^{\prime} \stackrel{g^{\prime} f^{\prime}}{\longrightarrow} Z^{\prime}\right)$,

$$
h^{\star}(\alpha \cdot \beta)=h^{\prime \star}(\alpha) \cdot h^{\star}(\beta) .
$$

Proof. We may assume that $\alpha \in \mathrm{HH}^{i}(X \stackrel{f}{\rightarrow} Y)$ and $\beta \in \mathrm{HH}^{j}(Y \stackrel{g}{\rightarrow} Z)$. Then 
what is asserted is commutativity of the border of the next diagram:

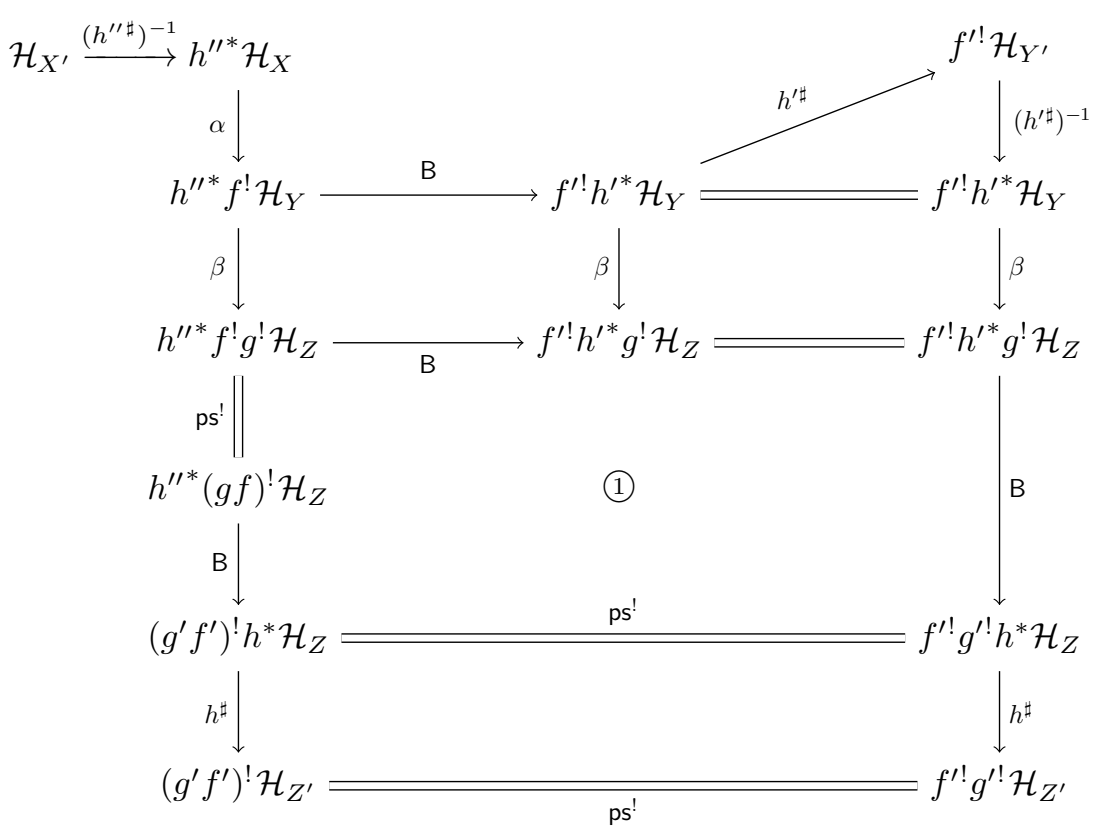

Subdiagram (1) commutes by (2.3.2); and commutativity of the other subdiagrams is clear. The desired result follows.

Proposition 4.6. $\left(A_{23}\right)$ Pushforward and pullback commute:

For any $\mathrm{S}$-diagram with independent squares and with $f$ confined,

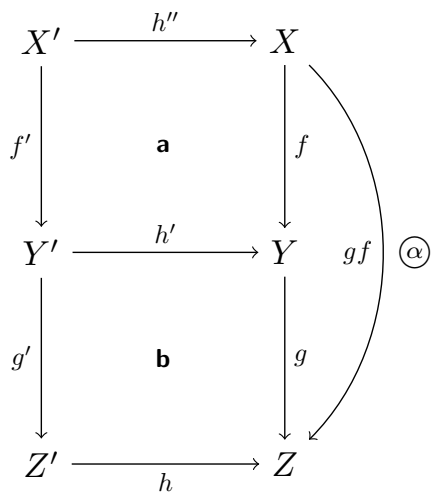

one has, in $\mathrm{HH}^{*}\left(Y^{\prime} \stackrel{g^{\prime}}{\rightarrow} Z^{\prime}\right)$,

$$
f_{\star}^{\prime}\left(h^{\star}(\alpha)\right)=h^{\star}\left(f_{\star}(\alpha)\right) .
$$


Proof. What is asserted is commutativity of the border of the following diagram, in which $\mathbf{c}$ denotes the square obtained by pasting $\mathbf{a}$ and $\mathbf{b}$ :

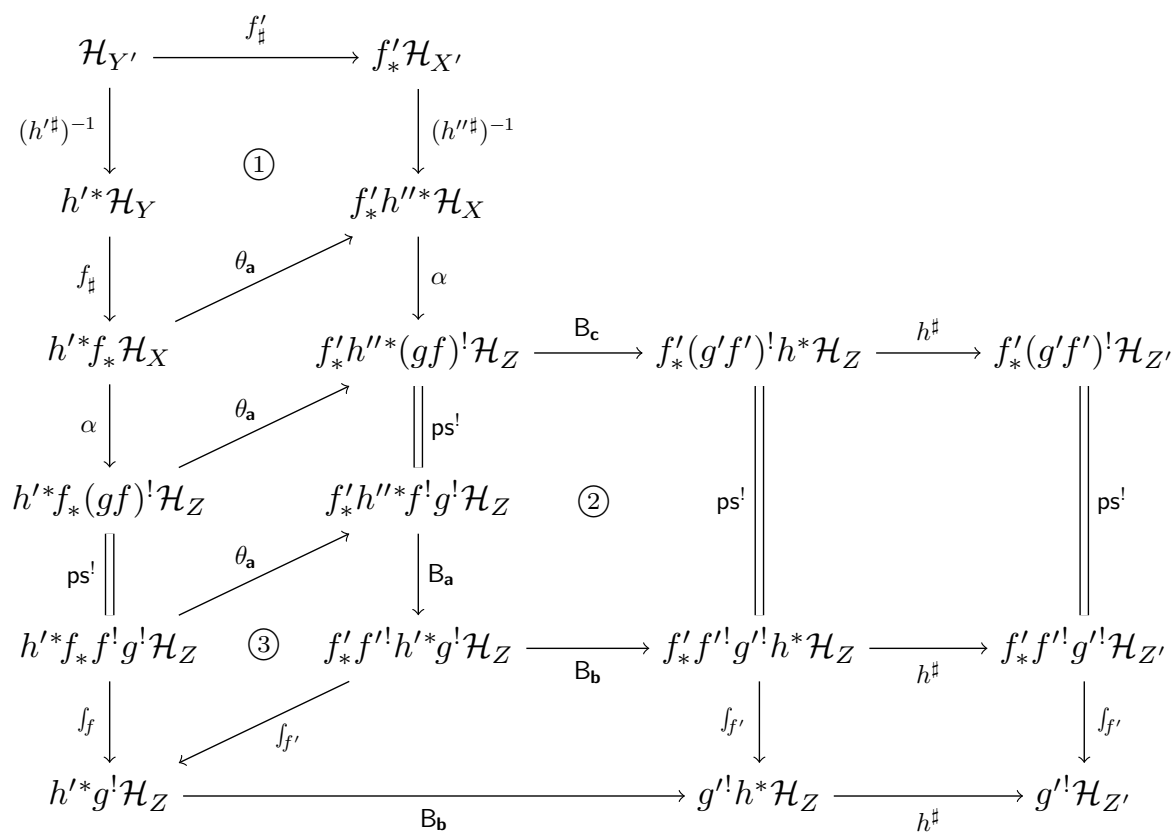

Commutativity of subdiagram (2) is given by (2.3.2), and of (3) by (2.6.1). Commutativity of the unlabeled subdiagrams is clear.

Commutativity of subdiagram (1) is equivalent to that of its adjoint, and so of the border of the following diagram, where $k:=h^{\prime} f^{\prime}=f h^{\prime \prime}$, so that commutativity of (4) and (5) results from (3.2.1), and where commutativity of the other subdiagrams results directly from the definitions of the maps involved.

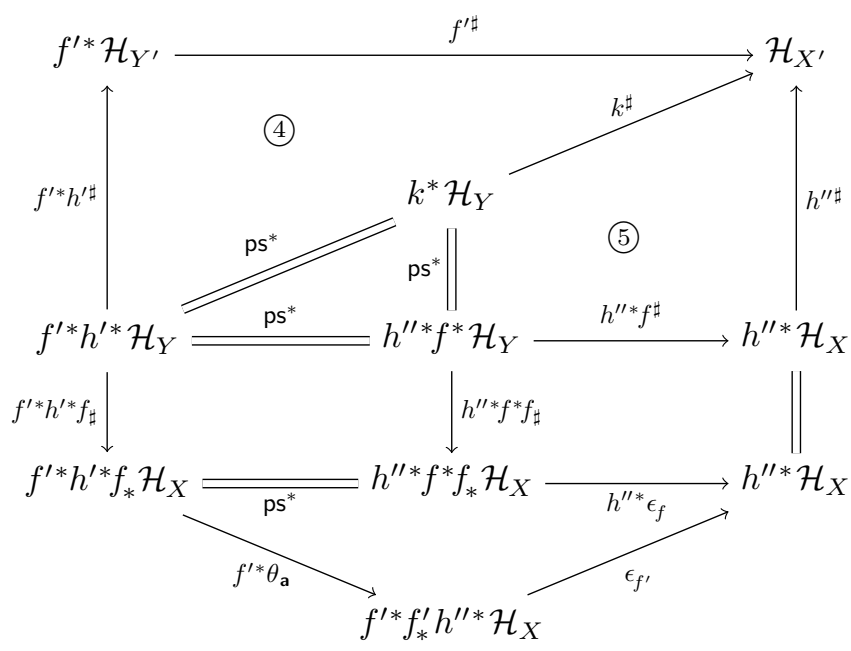

The desired result follows. 
Proposition 4.7. $\left(A_{123}\right)$ Projection formula:

For any S-diagram, with independent square and $g$ confined,

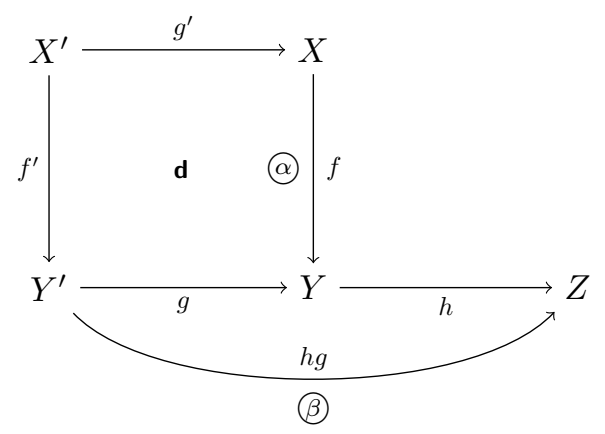

one has, in $\mathrm{HH}^{*}(X \stackrel{h f}{\longrightarrow} Z)$.

$$
g_{\star}^{\prime}\left(g^{\star} \alpha \cdot \beta\right)=\alpha \cdot g_{\star}(\beta)
$$

Proof. We may assume that $\alpha \in \mathrm{HH}^{i}(X \stackrel{f}{\rightarrow} Y)$ and $\beta \in \mathrm{HH}^{j}\left(Y^{\prime} \stackrel{h g}{\longrightarrow} Z\right)$. What is asserted is commutativity of the border of diagram (4.7.1) below, where commutativity of the unlabeled subdiagrams is obvious, and that of subdiagrams (1) and (2) holds by adjointness of $g^{\prime \sharp}$ and $g_{\sharp}^{\prime}$ (resp. $g^{\sharp}$ and $g_{\sharp}$ ). It remains then to show that (3) commutes.

Via the next, obviously commutative, diagram (in which $\mathcal{H}_{Z}$ is omitted),

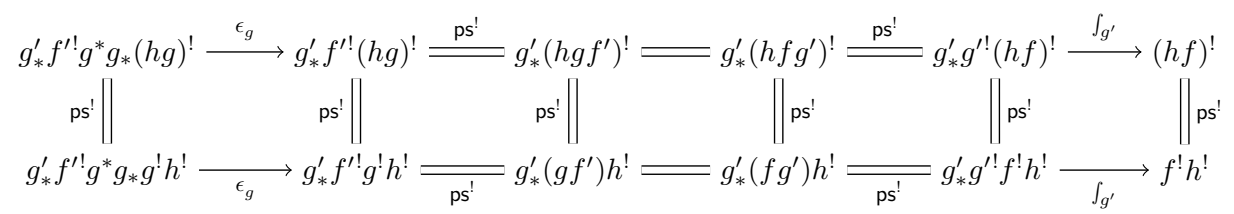

commutativity of (3) becomes equivalent to that of

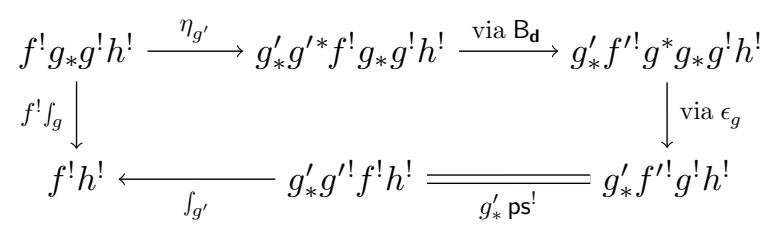

which commutativity is an instance of that of (2.6.2).

The proof of Proposition 4.7 is now complete. 
(4.7.1)

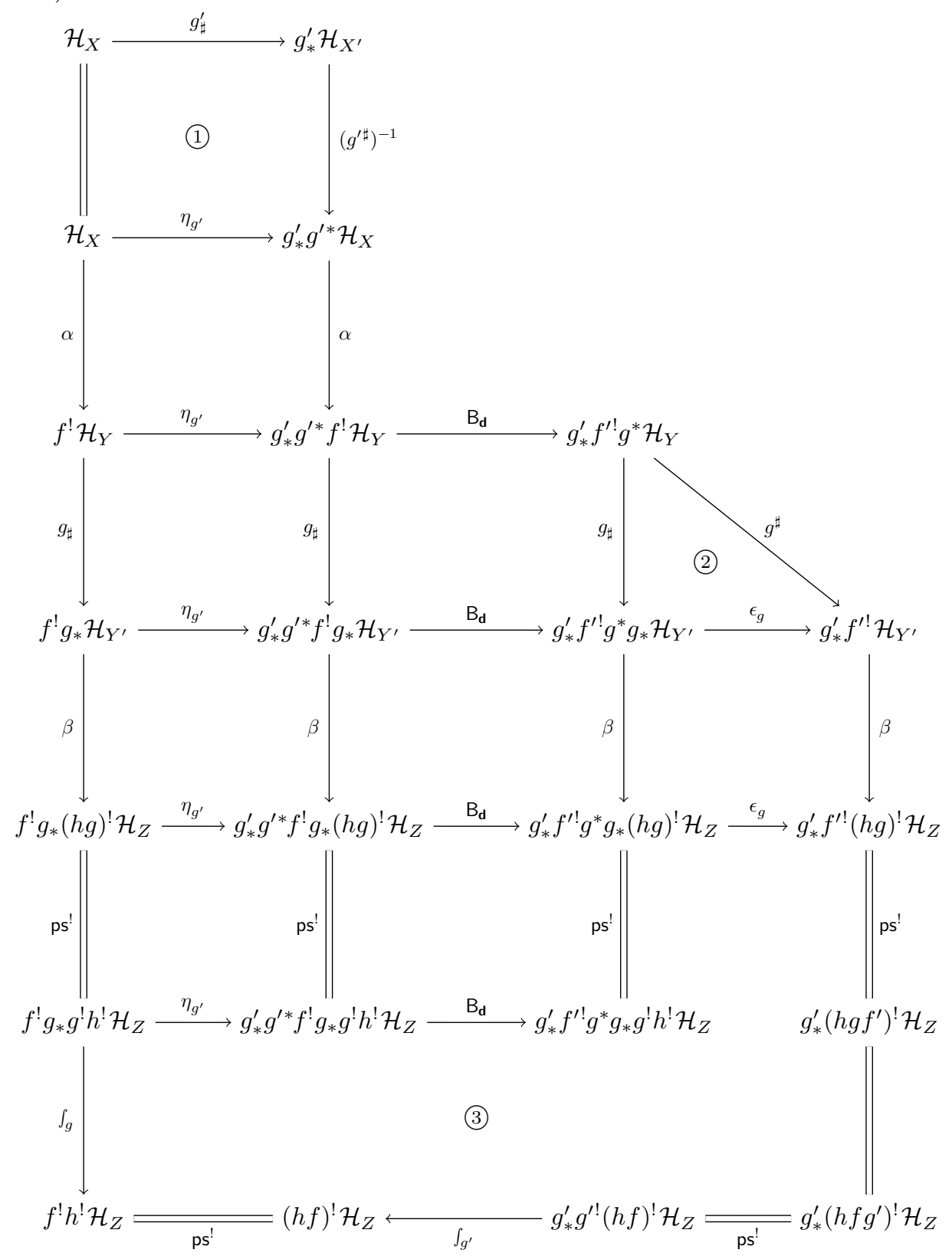

5. Realization via Grothendieck duality. In this section we show that the setup of $\S 2$ can be realized in a number of situations involving Grothendieck duality.

5.1. (Notation and summary.) A ringed space is a pair $\left(X, \mathcal{O}_{X}\right)$ such that $X$ is a topological space and $\mathcal{O}_{X}$ is a sheaf of commutative rings on $X$. Although only schemes will be of interest in this paper, some initial results make sense for arbitrary ringed spaces, enabling us to treat several situations simultaneously. For example, 
it may well be possible to go through all of this section in the context of noetherian formal schemes, see [AJL], [Nk1, 7.1.6].

A map of ringed spaces $\bar{f}:\left(X, \mathcal{O}_{X}\right) \rightarrow\left(Y, \mathcal{O}_{Y}\right)$ is a continuous map $f: X \rightarrow Y$ together with a homomorphism of sheaves of rings $\mathcal{O}_{Y} \rightarrow f_{*} \mathcal{O}_{X}$. Composition of such maps is defined in the obvious way. Ordinarily, $\mathcal{O}_{X}$ and $\mathcal{O}_{Y}$ are omitted from the notation, and one just speaks of ringed-space maps $f: X \rightarrow Y$, the rest being understood.

For a ringed space $\left(X, \mathcal{O}_{X}\right)$, let $\mathbf{D}(X)$ be the derived category of the abelian category of sheaves of $\mathcal{O}_{X}$-modules, and $T=T_{X}$ its usual translation automorphism. For $A \in \mathbf{D}(X)$ (object or arrow) and $i \in \mathbb{Z}$, set $A[i]:=T^{i} A$.

We take for granted the formalism of relations among the derived functors RHom and $\otimes^{\mathrm{L}}$ and the derived direct- and inverse-image pseudofunctors $\mathrm{R}(-)_{*}$ resp. $\mathrm{L}(-)^{*}$, as presented e.g., in [L3, Chapter 3]. ${ }^{1}$ For instance, for any $f: X \rightarrow Y$ as above, the functor $\mathbf{L} f^{*}: \mathbf{D}(Y) \rightarrow \mathbf{D}(X)$ is left-adjoint to $\mathrm{R} f_{*}$, see [L3, 3.2.3]; in particular, there are unit and counit maps

$$
\bar{\eta}=\bar{\eta}_{f}: \mathrm{id} \rightarrow \mathrm{R} f_{*} \mathrm{~L} f^{*}, \quad \bar{\epsilon}=\bar{\epsilon}_{f}: \mathrm{L} f^{*} \mathrm{R} f_{*} \rightarrow \mathrm{id} .
$$

For any $f: X \rightarrow Y$, there are canonical functorial isomorphisms

$$
\mathrm{R} f_{*} \circ T_{X} \stackrel{\sim}{\longrightarrow} T_{Y} \circ \mathrm{R} f_{*}, \quad \mathrm{~L} f^{*} \circ T_{Y} \stackrel{\sim}{\longrightarrow} T_{X} \circ \mathrm{L} f^{*} .
$$

Accordingly, for any $A \in \mathbf{D}(X), B \in \mathbf{D}(Y)$ and $i \in \mathbb{Z}$, we will allow ourselves to abuse notation by writing

$$
\mathrm{R} f_{*}(A[i])=\left(\mathrm{R} f_{*} A\right)[i], \quad \mathrm{L} f^{*}(B[i])=\left(\mathrm{L} f^{*} B\right)[i] .
$$

5.1.2. Let $\mathbf{E}_{X}$ be the preadditive category whose objects $A, B, C, \ldots$ are just those of $\mathbf{D}(X)$, with

$$
\mathbf{E}_{X}^{i}(A, B):=\operatorname{Hom}_{\mathbf{D}(X)}(A, B[i]) \cong \operatorname{Ext}_{X}^{i}(A, B),
$$

and composition determined by the graded $\mathbb{Z}$-bilinear Yoneda product

$$
\mathbf{E}_{X}^{i}(B, C) \times \mathbf{E}_{X}^{j}(A, B) \rightarrow \mathbf{E}_{X}^{i+j}(A, C)
$$

taking a pair of $\mathbf{D}(X)$-maps $\beta: B \rightarrow C[i], \alpha: A \rightarrow B[j](i, j \in \mathbb{Z})$ to the map

$$
(\beta \circ \alpha): A \stackrel{\alpha}{\longrightarrow} B[j] \stackrel{\beta[j]}{\longrightarrow} C[i][j]=C[i+j] .
$$

5.1.3. In subsection 5.2 , using their interaction with translation functors, we enrich the derived direct- and inverse-image pseudofunctors to an adjoint pair of $\mathbb{Z}$ graded pseudofunctors $(-)^{*}$ and $(-)_{*}$ on the category of ringed spaces, taking values in the categories $\mathbf{E}_{X}$.

\footnotetext{
${ }^{1}$ We will often use [L3] as a convenient compendium of needed facts about Grothendieck duality for schemes. This does not mean that referred-to results cannot be found in other earlier sources.
} 
Then we show in Proposition 5.4 that

$$
H_{X}:=\mathbf{E}_{X}\left(\mathcal{O}_{X}, \mathcal{O}_{X}\right)=\oplus_{i \geq 0} \operatorname{Ext}_{X}^{i}\left(\mathcal{O}_{X}, \mathcal{O}_{X}\right) \cong \oplus_{i \geq 0} \mathrm{H}^{i}\left(X, \mathcal{O}_{X}\right)
$$

with its Yoneda product is a commutative-graded ring, and that the category $\mathbf{E}_{X}$ is naturally $H_{X}$-graded - whence so is any full subcategory. In fact, Proposition 5.5 gives that $H_{X}$ can be identified with the subring of the graded center of $\mathbf{E}_{X}$ consisting of all "tensor-compatible" elements. Furthermore, Proposition 5.6.1 gives that for any map $f: X \rightarrow Y$, the functors $f^{*}$ and $f_{*}$ respect such graded structures.

5.1.4. A scheme-map $f: X \rightarrow Y$ is essentially of finite presentation if it is quasi-compact and quasi-separated, and for all $x \in X$ there exist affine open subsets Spec $L \supset\{x\}$ of $X$ and $\operatorname{Spec} K \supset f(\operatorname{Spec} L)$ of $Y$ such that the resulting $K$-algebra $L$ is a ring of fractions of a finitely-presentable $K$-algebra.

For maps of noetherian schemes, we use in place of "finite presentation" the equivalent term "finite type."

5.1.5. Now fix a scheme $S$, and let $\mathrm{S}$ be one of:

(a) The category of essentially-finite-type separated perfect (i.e., finite tordimension) maps of noetherian $S$-schemes, with proper maps as confined maps, and oriented fiber squares with flat bottom arrow as independent squares.

(b) The category of composites of étale maps and flat quasi-proper (equivalently, flat quasi-perfect) maps of arbitrary quasi-compact quasi-separated $S$-schemes (see $[\mathrm{L} 3, \S 4.7])$, with quasi-proper maps confined and all oriented fiber squares independent. (The reader who wishes to avoid the technicalities involved can safely ignore this case (b).)

Conditions (A1), (A2), (B1), (B2) and (C) in $\S 2.1$ are then easily checked.

As is customary, we will usually denote an object $W \stackrel{w}{\rightarrow} S$ in S simply by $W$, with the understanding that $W$ is equipped with a "structure map" $w$.

For any such $W$, let $\mathbf{D}_{W}$ be the full subcategory of $\mathbf{E}_{W}$ whose objects are just those of $\mathbf{D}_{\mathrm{qc}}(W)$, that is, $\mathcal{O}_{X}$-complexes whose homology sheaves are all quasicoherent. Since for $f: X \rightarrow Y$ in $\mathrm{S}$ it holds that $\mathrm{L} f^{*} \mathbf{D}_{\mathrm{qc}}(Y) \subset \mathbf{D}_{\mathrm{qc}}(X)[\mathrm{L} 3,3.9 .1]$ and $\mathrm{R} f_{*} \mathbf{D}_{\mathrm{qc}}(X) \subset \mathbf{D}_{\mathrm{qc}}(Y)[\mathrm{L} 3,3.9 .2]$, it follows that the pseudofunctors $(-)^{*}$ and $(-)_{*}$ in 5.1.3 can be restricted to take values in the categories $\mathbf{D}_{W}$. It is assumed henceforth that they are so restricted.

Let $H$ be the commutative-graded ring $H_{S}:=\mathbf{E}_{S}\left(\mathcal{O}_{S}, \mathcal{O}_{S}\right)$. For any S-object $w: W \rightarrow S$, the natural composite map

$$
\mathbf{E}_{S}\left(\mathcal{O}_{S}, \mathcal{O}_{S}\right) \rightarrow \mathbf{E}_{W}\left(w^{*} \mathcal{O}_{S}, w^{*} \mathcal{O}_{S}\right) \stackrel{\sim}{\longrightarrow} \mathbf{E}_{W}\left(\mathcal{O}_{W}, \mathcal{O}_{W}\right)
$$

is a graded-ring homomorphism from $H_{S}$ to $H_{W}$. Hence $\mathbf{D}_{W}$ is $H$-graded, and the adjoint pseudofunctors $(-)^{*}$ and $(-)_{*}$ are $H$-graded, see 5.1.3.

We note in Proposition 5.2.4 that for an independent square d, the associated functorial map $\theta_{\mathbf{d}}(\S 2.5)$ is a degree-0 isomorphism.

Thus, we have in place all those elements of a setup that do not involve the pseudofunctor $(-)^{!}$.

5.1.6. In subsections $5.7-5.10$, we treat those elements involving $(-)^{!}$by using the twisted inverse-image pseudofunctor from Grothendieck duality. The twisted inverse image is generally defined only for bounded-below complexes. But we want a 
pseudofunctor with values on all of $\mathbf{D}_{W}$. (For instance, we have in mind Hochschild homology, which involves complexes that are bounded above, not below.) That is why we restrict in the examples 5.1.5(a) and (b) to maps of finite tor-dimension: the twisted inverse image functor $f_{+}^{!}$that is attached to such a map $f: X \rightarrow Y$ extends to a functor $f^{!}: \mathbf{D}_{\mathrm{qc}}(Y) \rightarrow \mathbf{D}_{\mathrm{qc}}(X)$ with

$$
f^{!} C:=f_{+}^{!} \mathcal{O}_{Y} \otimes_{X}^{\llcorner} \mathrm{L} f^{*} C \quad\left(C \in \mathbf{D}_{\mathrm{qc}}(Y)\right) .
$$

"Extends" means that for cohomologically bounded-below $C \in \mathbf{D}_{\mathrm{qc}}(Y)$, there is a canonical functorial isomorphism

$$
f^{!} C \stackrel{\sim}{\longrightarrow} f_{+}^{!} C .
$$

(For case (a), see [Nk2, 5.9]; for (b), [L3, 4.7.2]). This extension can be made pseudofunctorial ( $\S 5.7)$, and $H$-graded, the latter as a consequence of the compatibility of $\otimes_{X}^{L}$ and $\mathbf{L} f^{*}$ with the $H_{X}$-grading on $\mathbf{E}_{X}$ (Propositions 5.5 and 5.6.1).

In $\S 5.8$ we associate to each independent square $\mathbf{d}$ an isomorphism $\boldsymbol{B}_{\mathbf{d}}$ as in $\S 2.3$, for which the diagrams (2.3.1) and (2.3.2) commute. In $\S 5.9$, we associate to each confined map $f$ a degree-0 functorial map $\int_{f}: f_{*} f^{!} \rightarrow$ id that satisfies transitivity (see $\S 2.4)$.

We conclude by showing that with the preceding data, diagrams (2.6.1) and (2.6.2) commute, thereby establishing all the properties of a setup.

5.2. Let $f: X \rightarrow Y$ be a ringed-space map. For any object $C$ in $\mathbf{E}_{Y}$, denote the derived inverse image $\mathrm{L} f^{*} C \in \mathbf{E}_{X}$ simply by $f^{*} C$. (Despite this notation, it should not be forgotten that we will be dealing throughout with derived functors.) To any map $\gamma: C \rightarrow D[i]$ in $\mathbf{E}_{Y}^{i}(C, D)$ assign the map

$$
f^{*} \gamma: f^{*} C \stackrel{\left\llcorner f^{*} \gamma\right.}{\longrightarrow} f^{*}(D[i])=\left(f^{*} D\right)[i]
$$

in $\mathbf{E}_{X}^{i}\left(f^{*} C, f^{*} D\right)$. Using functoriality of the isomorphism represented by "=" (see §5.1), one checks that this assignment is compatible with composition in $\mathbf{E}_{Y}$ and $\mathbf{E}_{X}$; so one gets a $\mathbb{Z}$-graded functor $f^{*}: \mathbf{E}_{Y} \rightarrow \mathbf{E}_{X}$.

In a similar manner, the derived direct image functor $\mathrm{R} f_{*}$ gives rise to a $\mathbb{Z}$-graded functor $f_{*}: \mathbf{E}_{X} \rightarrow \mathbf{E}_{Y}$.

Proposition 5.2.1. There is an adjunction $f^{*} \dashv f_{*}$ for which the corresponding unit and counit maps

$$
\eta: \text { id } \rightarrow f_{*} f^{*} \quad \text { and } \quad \epsilon: f^{*} f_{*} \rightarrow \text { id }
$$

are degree-0 maps of $\mathbb{Z}$-graded functors.

Proof. Let $\eta_{C} \in \mathbf{E}_{Y}^{0}\left(C, f_{*} f^{*} C\right)$ be the $\mathbf{D}(Y)$-map $\bar{\eta}_{C}: C \rightarrow f_{*} f^{*} C$ (see (5.1.1)) and $\epsilon_{A} \in \mathbf{E}_{X}^{0}\left(f^{*} f_{*} A, A\right)$ the $\mathbf{D}(X)$-map $\bar{\epsilon}_{A}: f^{*} f_{*} A \rightarrow A$.

That the compositions

$$
f_{*} A \stackrel{\eta_{f_{*} A}}{\longrightarrow} f_{*} f^{*} f_{*} A \stackrel{f_{*} \epsilon_{A}}{\longrightarrow} f_{*} A, \quad f^{*} C \stackrel{f^{*} \eta_{C}}{\longrightarrow} f^{*} f_{*} f^{*} C \stackrel{\epsilon_{f^{*} C}}{\longrightarrow} f^{*} C
$$

are identity maps follows from the corresponding properties of $\bar{\eta}$ and $\bar{\epsilon}$. It remains then to show that the family $\eta_{C}\left(C \in \mathbf{E}_{Y}\right)$ (resp. $\left.\epsilon_{A}\left(A \in \mathbf{E}_{X}\right)\right)$ constitutes a degree-0 map 
of graded functors. For $\eta_{C}$ this means that for any $\mathbf{D}(Y)$-map $\gamma: C \rightarrow D[i](i \in \mathbb{Z})$ the following $\mathbf{D}(Y)$-diagram commutes:

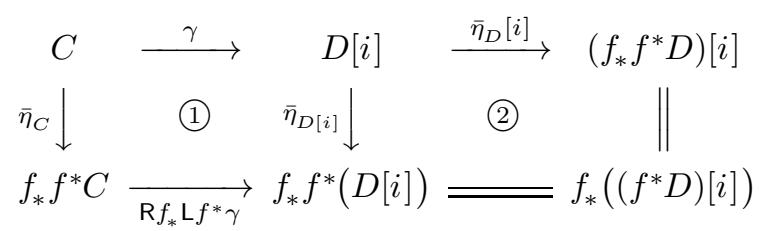

Commutativity of subdiagram (1) is clear.

For commutativity of (2), replace $D$ by a quasi-isomorphic q-flat complex, and note that the natural map from the derived inverse image to the underived inverse image of $D$ is then an isomorphism, see [L3, paragraph surrounding 2.7.3.1]. Then, with $\tilde{f}_{*}$ denoting the underived direct-image functor, consider the following cube, in which the front face is (2) and the maps are the natural ones:

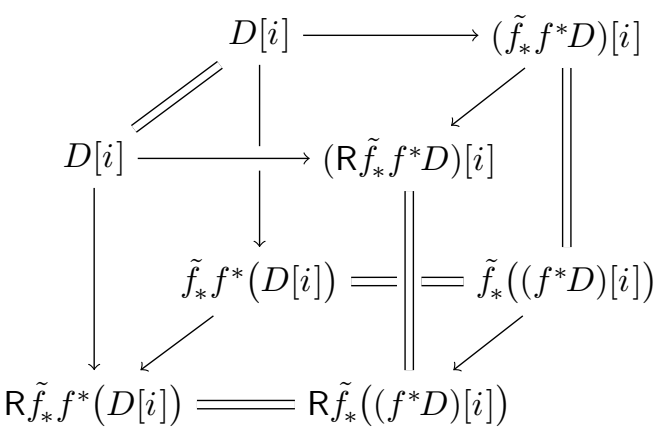

Commutativity of the bottom face is clear. Commutativity of the top and left faces results from [L3, 3.2.1.3]. To make commutativity of the right face clear, replace the complex $f^{*} D$ by a quasi-isomorphic q-injective complex $J$, and note that the canonical map $\tilde{f}_{*} J \rightarrow \mathrm{R} \tilde{f}_{*} J$ is a $\mathbf{D}(Y)$-isomorphism (see $[\mathrm{L} 3,2.3 .5]$ ). Commutativity of the rear face, which involves only underived functors, is an easy consequence of the definition of the standard functorial map id $\rightarrow \tilde{f}_{*} f^{*}$. Commutativity of the front face follows from that of the others.

An analogous argument, using [L3, 3.2.1.2], applies to the family $\epsilon_{A}$. $\square$

COROLlary 5.2.3. There exist pseudofunctorially adjoint $\mathbb{Z}$-graded pseudofunctors that associate the functors $f^{*}$ and $f_{*}$ to any S-map $f: X \rightarrow Y$.

Proof. For any $X \stackrel{f}{\rightarrow} Y \stackrel{g}{\rightarrow} Z$ in S, there are functorial isomorphisms

$$
\mathrm{ps}_{*}:(g f)_{*} \stackrel{\sim}{\longrightarrow} g_{*} f_{*}, \quad \mathrm{ps}^{*}: f^{*} g^{*} \stackrel{\sim}{\longrightarrow}(g f)^{*}
$$

such that for $A \in \mathbf{E}_{X}, \mathrm{ps}_{*}(A):(g f)_{*} A \stackrel{\sim}{\longrightarrow} g_{*} f_{*} A$ is the $\mathbf{D}(Z)$-isomorphism $\overline{\mathrm{ps}}_{*}(A): \mathrm{R}(g f)_{*} A \stackrel{\sim}{\longrightarrow} \mathrm{R}_{*} \mathrm{R} f_{*} A$, and for $C \in \mathbf{E}_{Z}, \mathrm{ps}^{*}(C): f^{*} g^{*} C \stackrel{\sim}{\longrightarrow}(g f)^{*} C$ is the $\mathbf{D}(X)$-isomorphism $\overline{\mathrm{ps}}^{*}(C): \mathrm{L} f^{*} \mathrm{~L} g^{*} C \stackrel{\sim}{\longrightarrow} \mathrm{L}(g f)^{*} C$.

That the first diagram in $(2.2 .1)$ commutes, as does its analog for $(-)_{*}$, follows from the corresponding facts for the pseudofunctors $\mathrm{L}(-)^{*}$ and $\mathrm{R}(-)_{*}$. Consequently, $\mathrm{ps}^{*}$ makes $(-)^{*}$ into a contravariant $\mathbb{Z}$-graded pseudofunctor, and $\mathrm{ps}_{*}$ makes $(-)_{*}$ into a covariant $\mathbb{Z}$-graded pseudofunctor. The adjointness of these pseudofunctors, that 
is, commutativity of (2.4.2), results from that of the corresponding diagram for the adjoint pseudofunctors $\mathrm{L}(-)^{*}$ and $\mathrm{R}(-)_{*}$ (see $[\mathrm{L} 3,3.6 .10]$ ).

From [L3, 3.9.5]), one gets:

Proposition 5.2.4. With $f^{*} \dashv f_{*}$ as above, for any independent S-square

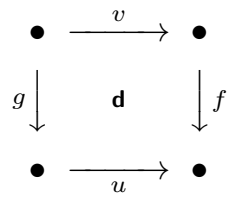

the map $\theta_{\mathbf{d}}: u^{*} f_{*} \rightarrow g_{*} v^{*}$ in $\S 2.5$ is a functorial isomorphism of degree 0 .

Proof. That $\theta_{\mathbf{d}}$ has degree 0 results from the fact that it is a composition of three functorial maps

$$
u^{*} f_{*} \stackrel{\eta_{g}}{\longrightarrow} g_{*} g^{*} u^{*} f_{*} \stackrel{\mathrm{ps}^{*}}{=} g_{*} v^{*} f^{*} f_{*} \stackrel{\epsilon_{f}}{\longrightarrow} g_{*} v^{*}
$$

all of which are of degree 0 (see 5.2.1 and the proof of 5.2.3).

The rest is clear.

5.3. For a scheme $\left(X, \mathcal{O}_{X}\right)$, if $A$ and $B$ are $\mathcal{O}_{X}$-complexes and $i, j, n \in \mathbb{Z}$, then since

$$
\left(A[i] \otimes_{X} B[j]\right)^{n}=\bigoplus_{p+q=n+i+j} A^{p} \otimes_{X} B^{q}=\left(A \otimes_{X} B\right)[i+j]^{n},
$$

therefore there is a unique isomorphism of graded $\mathcal{O}_{X}$-modules

$$
\vartheta_{i j}^{\prime}: A[i] \otimes_{X} B[j] \stackrel{\sim}{\longrightarrow}\left(A \otimes_{X} B\right)[i+j]
$$

whose restriction to $A^{p} \otimes_{X} B^{q}(p, q \in \mathbb{Z})$ is multiplication by $(-1)^{(p-i) j}$. One checks that $\vartheta_{i j}^{\prime}$ is actually a bifunctorial isomorphism of $\mathcal{O}_{X}$-complexes.

LEMma 5.3.1. For any $i, j \in \mathbb{Z}$ there exists a unique bifunctorial isomorphism $\vartheta_{i j}$ such that for any $\mathcal{O}_{X}$-complexes $A$ and $B$, the following diagram in $\mathbf{D}(X)$ commutes.

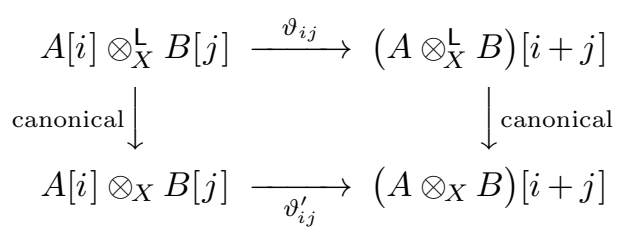

Proof. The idea is to apply $\vartheta_{i j}^{\prime}$ to suitable q-flat resolutions of $A$ and $B$.

More precisely, every $\mathcal{O}_{X}$-complex is the target of a quasi-isomorphism from a q-flat complex, and for q-flat complexes the canonical functorial map from the derived tensor product $\otimes_{X}^{\mathrm{L}}$ to the ordinary tensor product $\otimes_{X}$ is an isomorphism [L3, $\S 2.5]$; hence the assertion follows from [L3, 2.6.5] (a general method for constructing maps of derived multifunctors), dualized - i.e., with arrows reversed, in which, with abbreviated notation, take

- $L_{1}^{\prime \prime}=L_{2}^{\prime \prime}$ to be the homotopy category $\mathbf{K}(X)$ of $\mathcal{O}_{X}$-complexes, 
- $L_{k}^{\prime} \subset L_{k}^{\prime \prime}(k=1,2)$ the full subcategory whose objects are the q-flat complexes,

- $\mathbf{E}:=\mathbf{D}(X)$,

- $H$ the functor taking $(A, B) \in L_{1}^{\prime \prime} \times L_{2}^{\prime \prime}$ to $\left(A \otimes_{X} B\right)[i+j] \in \mathbf{D}(X)$ (and acting in the obvious way on arrows),

- $G$ the functor $(A, B) \in \mathbf{D}(X) \times \mathbf{D}(X) \mapsto A[i] \otimes_{X}^{\mathrm{L}} B[j] \in \mathbf{D}(X)$,

- $F$ the functor $(A, B) \in \mathbf{D}(X) \times \mathbf{D}(X) \mapsto\left(A \otimes_{X}^{\mathrm{L}} B\right)[i+j] \in \mathbf{D}(X)$,

- $\zeta: F \rightarrow H$ the canonical functorial map, and

- $\beta: G \rightarrow H$ the canonical functorial composite

$$
A[i] \otimes_{X}^{\llcorner} B[j] \longrightarrow A[i] \otimes_{X} B[j] \stackrel{\vartheta_{i j}^{\prime}}{\longrightarrow}\left(A \otimes_{X} B\right)[i+j] .
$$

Proposition 5.4. The ring

$$
H_{X}:=\mathbf{E}_{X}\left(\mathcal{O}_{X}, \mathcal{O}_{X}\right)=\oplus_{i \geq 0} \operatorname{Ext}_{X}^{i}\left(\mathcal{O}_{X}, \mathcal{O}_{X}\right) \cong \oplus_{i \geq 0} \mathrm{H}^{i}\left(X, \mathcal{O}_{X}\right)
$$

is canonically a graded-ring retract of the graded center $\mathcal{C}_{\mathbf{E}_{X}}$. Hence $H_{X}$ is gradedcommutative, and $\mathbf{E}_{X}$ is $H_{X}$-graded.

Proof. By $\$ 1.4 .3$, the assertion follows from the existence of a unital product $\left(\bar{\otimes}, \mathcal{O}_{X}, \lambda, \rho\right)$ - to be constructed - on the preadditive category $\mathbf{E}_{X}$.

Define a $\mathbb{Z}$-graded functor

$$
\bar{\otimes}: \mathbf{E}_{X} \otimes_{\mathbb{Z}} \mathbf{E}_{X} \rightarrow \mathbf{E}_{X}
$$

as follows. (Notation will be as in $\S 1.4$.)

First, for any object $(A, B) \in \mathbf{E}_{X} \otimes_{\mathbb{Z}} \mathbf{E}_{X}, A \bar{\otimes} B:=\bar{\otimes}(A, B)$ is the derived tensor product $A \otimes_{X}^{\mathrm{L}} B$, which lies in $\mathbf{E}_{X}$ [L3, p. 64, 2.5.8.1].

Next, the map taking $\left(\alpha_{1}, \alpha_{2}\right) \in \mathbf{E}_{X}^{i}\left(A_{1}, B_{1}\right) \times \mathbf{E}_{X}^{j}\left(A_{2}, B_{2}\right)$ to the map

$$
\alpha_{1} \bar{\otimes} \alpha_{2} \in \mathbf{E}_{X}^{i+j}\left(A_{1} \bar{\otimes} A_{2}, B_{1} \bar{\otimes} B_{2}\right)
$$

given by the composite $\mathbf{D}(X)$-map

$$
A_{1} \otimes_{X}^{\mathrm{L}} A_{2} \stackrel{\alpha_{1} \otimes_{X}^{\mathrm{L}} \alpha_{2}}{\longrightarrow} B_{1}[i] \otimes_{X}^{\mathrm{L}} B_{2}[j] \underset{(5.3 .1)}{\stackrel{\vartheta_{i j}}{\longrightarrow}}\left(B_{1} \otimes_{X}^{\mathrm{L}} B_{2}\right)[i+j]
$$

is $\mathbb{Z}$-bilinear, so factors uniquely through a map

$$
\bar{\otimes}^{i j}: \mathbf{E}_{X}^{i}\left(A_{1}, B_{1}\right) \otimes_{\mathbb{Z}} \mathbf{E}_{X}^{j}\left(A_{2}, B_{2}\right) \rightarrow \mathbf{E}_{X}^{i+j}\left(A_{1} \bar{\otimes} A_{2}, B_{1} \bar{\otimes} B_{2}\right)
$$

taking $\alpha_{1} \otimes \alpha_{2}$ to $\alpha_{1} \bar{\otimes} \alpha_{2}$; and $\bar{\otimes}^{i j}$ extends uniquely to a $\mathbb{Z}$-linear map

$$
\begin{aligned}
\bar{\otimes}:\left(\mathbf{E}_{X} \otimes_{\mathbb{Z}} \mathbf{E}_{X}\right)\left(\left(A_{1}, A_{2}\right),\right. & \left.\left(B_{1}, B_{2}\right)\right)= \\
& \mathbf{E}_{X}\left(A_{1}, B_{1}\right) \otimes_{\mathbb{Z}} \mathbf{E}_{X}\left(A_{2}, B_{2}\right) \rightarrow \mathbf{E}_{X}\left(A_{1} \bar{\otimes} A_{2}, B_{1} \bar{\otimes} B_{2}\right) .
\end{aligned}
$$

For functoriality, it needs to be checked that for all $A_{1} \stackrel{\alpha_{1}}{\longrightarrow} B_{1} \stackrel{\beta_{1}}{\longrightarrow} C_{1}$ and $A_{2} \stackrel{\alpha_{2}}{\longrightarrow} B_{2} \stackrel{\beta_{2}}{\longrightarrow} C_{2}$ in $\mathbf{E}_{X}$, with $\alpha_{1} \in \mathbf{E}_{X}^{m_{1}}\left(A_{1}, B_{1}\right)$ and $\beta_{2} \in \mathbf{E}_{X}^{n_{2}}\left(B_{2}, C_{2}\right)$, it holds that

$$
\left(\beta_{1} \otimes \beta_{2}\right) \circ\left(\alpha_{1} \otimes \alpha_{2}\right)=(-1)^{n_{2} m_{1}}\left(\beta_{1} \circ \alpha_{1}\right) \otimes\left(\beta_{2} \circ \alpha_{2}\right): A_{1} \otimes A_{2} \rightarrow C_{1} \otimes C_{2} .
$$


This straightforward verification is left to the patient reader.

Specializing, one gets the $\mathbb{Z}$-graded endofunctor $\mathcal{O}_{X} \bar{\otimes}-$ of $\mathbf{E}_{X}$, taking an object $A \in \mathbf{E}_{X}$ to $\mathcal{O}_{X} \otimes_{X}^{\llcorner} A$, and a $\mathbf{D}(X)$-map $\alpha: A \rightarrow B[j]$ in $\mathbf{E}_{X}^{j}(A, B)$ to the composite $\mathbf{D}(X)$-map, in $\mathbf{E}_{X}^{j}\left(\mathcal{O}_{X} \bar{\otimes} A, \mathcal{O}_{X} \bar{\otimes} B\right)$,

$$
\mathcal{O}_{X} \otimes_{X}^{\mathrm{L}} A \stackrel{\text { via } \alpha}{\longrightarrow} \mathcal{O}_{X} \otimes_{X}^{\mathrm{L}} B[j] \stackrel{\vartheta_{0 j}}{=}\left(\mathcal{O}_{X} \otimes_{X}^{\mathrm{L}} B\right)[j]
$$

Similarly, one has the $\mathbb{Z}$-graded endofunctor $-\bar{\otimes} \mathcal{O}_{X}$. There are obvious degree- 0 functorial isomorphisms

$$
\lambda:\left(\mathcal{O}_{X} \bar{\otimes}-\right) \stackrel{\sim}{\longrightarrow} \operatorname{id}_{\mathbf{E}_{X}}, \quad \rho:\left(-\bar{\otimes} \mathcal{O}_{X}\right) \stackrel{\sim}{\longrightarrow} \operatorname{id}_{\mathbf{E}_{X}} .
$$

It is immediate that $\left(\bar{\otimes}, \mathcal{O}_{X}, \lambda, \rho\right)$ is a unital product, so we are done.

Corollary 5.4.2. Any full subcategory of $\mathbf{E}_{X}$ has an $H_{X}$-grading, inherited from the preceding one on $\mathbf{E}_{X}$.

The preceding $\mathbb{Z}$-graded unital product is in fact $H_{X}$-graded. This results from the following characterization of $H_{X} \subset \mathcal{C}_{\mathbf{E}_{X}}$.

PROPOSITION 5.5. With notation as in 5.4 and its proof, the following conditions on $\xi \in \mathcal{C}_{\mathbf{E}_{X}}^{n}$ are equivalent:

(i) $\xi \in H_{X}^{n}=H^{n}\left(X, \mathcal{O}_{X}\right)$.

(ii) For all $(\alpha, \beta) \in \mathbf{E}_{X}^{i}(A, C) \times \mathbf{E}_{X}^{j}(B, D)$, it holds that

$$
(\xi \alpha) \bar{\otimes} \beta=\xi(\alpha \bar{\otimes} \beta), \quad \alpha \bar{\otimes}(\beta \xi)=(\alpha \bar{\otimes} \beta) \xi, \quad \text { and } \quad(\alpha \xi) \bar{\otimes} \beta=\alpha \bar{\otimes}(\xi \beta) .
$$

Proof. (i) $\Rightarrow$ (ii). Since

$$
(\xi \alpha) \bar{\otimes} \beta=\left(\xi_{C} \bar{\otimes} \operatorname{id}_{D}\right) \circ(\alpha \bar{\otimes} \beta) \quad \text { and } \quad \xi(\alpha \bar{\otimes} \beta)=\left(\xi_{C \bar{\otimes} D}\right) \circ(\alpha \bar{\otimes} \beta)
$$

therefore, for the first equality, one need only show that

$$
\left(\xi_{C} \bar{\otimes} \operatorname{id}_{D}\right)=\xi_{C \bar{\otimes} D}
$$

Similarly, the second equality reduces to

$$
\left(\mathrm{id}_{C} \bar{\otimes} \xi_{D}\right)=\xi_{C \bar{\otimes} D} .
$$

The third equality results from the first two, since the hom-sets $\mathbf{E}_{X}(-,-)$ are symmetric graded $\mathcal{C}_{\mathrm{E}_{X}}^{n}$-modules.

Thus, one need only treat the case where $\alpha: A=C \rightarrow C$ and $\beta: B=D \rightarrow D$ are the identity maps $\mathrm{id}_{C}$ and $\mathrm{id}_{D}$ respectively.

The equality (5.5.1) is equivalent to the obvious commutativity of the natural 
$\mathbf{D}(X)$-diagram, where $\otimes:=\otimes_{X}^{\mathrm{L}}$,

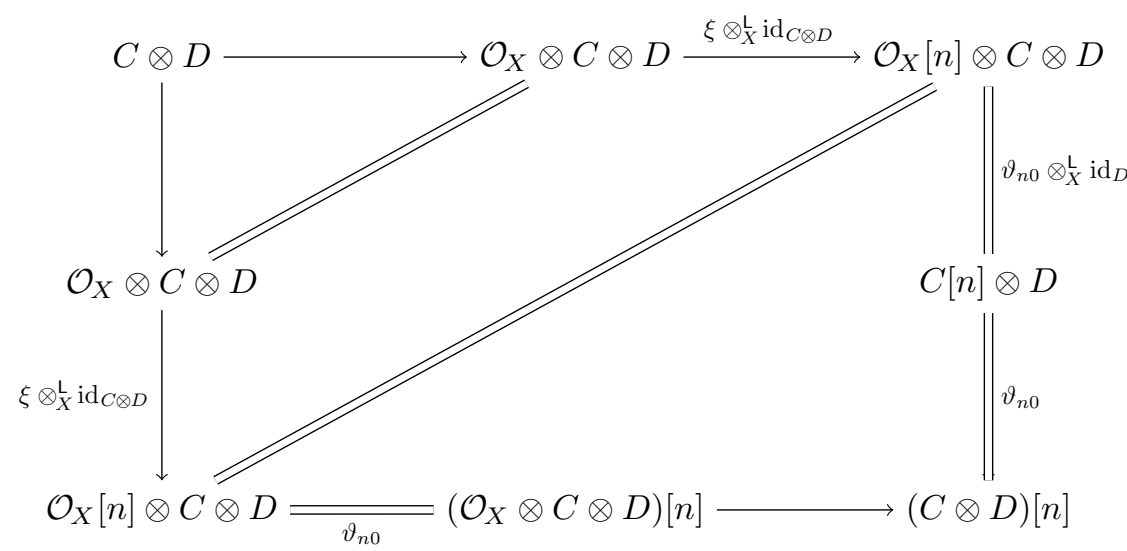

As for (5.5.2), let $\tau^{\prime}(A, B): A \otimes_{X} B \stackrel{\sim}{\longrightarrow} B \otimes_{X} A$ be the unique bifunctorial isomorphism of $\mathcal{O}_{X}$-complexes that restricts on $A^{p} \otimes_{X} B^{q}$ to the map taking $a \otimes b$ to $(-1)^{p q}(b \otimes a) \in B^{q} \otimes A^{p}(p, q \in \mathbb{Z})$. One shows as in Lemma 5.3.1 that there is a unique bifunctorial $\mathbf{D}(X)$-isomorphism $\tau(A, B)$ such that for any $A$ and $B$ the following $\mathbf{D}(X)$-diagram commutes:

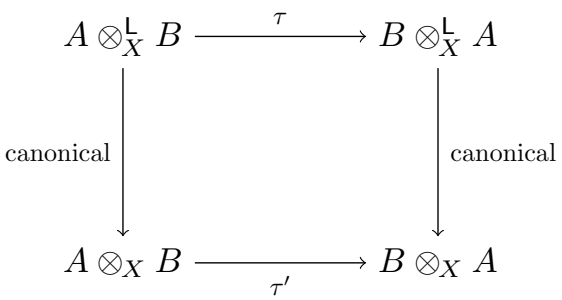

Equality (5.5.2) is equivalent to commutativity of the border of the natural diagram

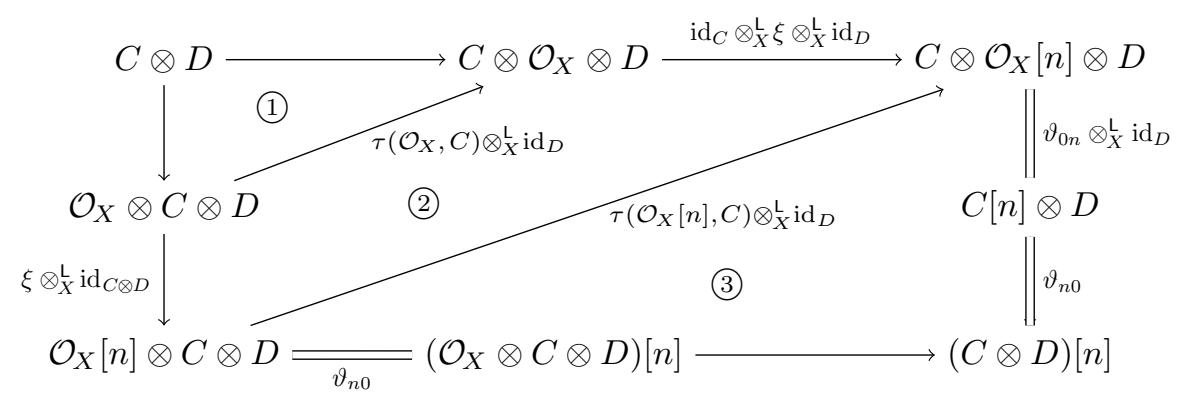

Commutativity of subdiagram (1) is easily checked. Commutativity of (2) holds by functoriality of $\tau$. For commutativity of (3), one checks, taking signs into account, that both paths from $\mathcal{O}_{X}[n] \otimes C \otimes D$ to $(C \otimes D)[n]$ have the same restriction to each $\mathcal{O}_{X}[n] \otimes C^{p} \otimes D^{q}(p, q \in \mathbb{Z})$.

The desired conclusion results.

(ii) $\Rightarrow$ (i). For $\alpha=\operatorname{id}_{\mathcal{O}_{X}} \in \mathbf{E}_{X}^{0}\left(\mathcal{O}_{X}, \mathcal{O}_{X}\right)$ and $\beta=\operatorname{id}_{A} \in \mathbf{E}_{X}^{0}(A, A)$ the identity maps, the third equality in condition (ii) yields

$$
\xi_{\mathcal{O}_{X}} \bar{\otimes} \operatorname{id}_{A}=\operatorname{id}_{\mathcal{O}_{X}} \bar{\otimes} \xi_{A} .
$$


In other words, in the following $\mathbf{D}(X)$-diagram-where unlabeled arrows represent the natural isomorphisms - subdiagram (4) commutes:

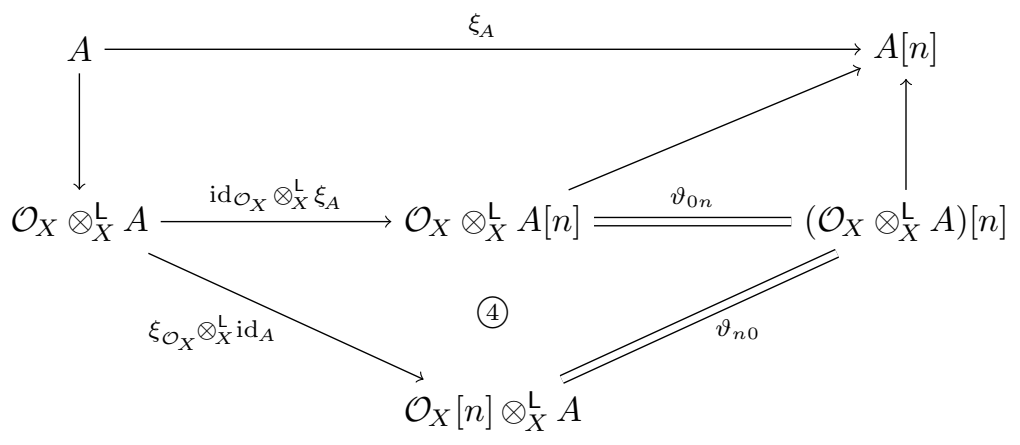

The other two subdiagrams clearly commute, so the border commutes. But by definition, the counterclockwise path from the upper left corner to the upper right corner is $\xi_{A}^{\prime}$, where $\xi^{\prime}$ is the canonical image in $\mathcal{C}_{\mathbf{E}_{X}}^{n}$ of the element $\xi_{\mathcal{O}_{X}} \in \mathbf{E}_{X}^{n}\left(\mathcal{O}_{X}, \mathcal{O}_{X}\right)=$ $H^{n}\left(X, \mathcal{O}_{X}\right)$. Thus, after identification of $H^{n}\left(X, \mathcal{O}_{X}\right)$ with its image in $\mathcal{C}_{\mathbf{E}_{X}}^{n}$, we have $\xi=\xi^{\prime} \in H^{n}\left(X, \mathcal{O}_{X}\right)$.

5.6. Let $f: X \rightarrow Y$ be a ringed-space map. The natural composition

$$
\mu_{f}: \mathbf{E}_{Y}\left(\mathcal{O}_{Y}, \mathcal{O}_{Y}\right) \rightarrow \mathbf{E}_{X}\left(f^{*} \mathcal{O}_{Y}, f^{*} \mathcal{O}_{Y}\right) \stackrel{\sim}{\longrightarrow} \mathbf{E}_{X}\left(\mathcal{O}_{X}, \mathcal{O}_{X}\right)
$$

is a graded-ring homomorphism from $H_{Y}$ to $H_{X}$. Hence, from 5.4.2, one gets an $H_{Y}$-grading on any full subcategory of $\mathbf{E}_{X}$.

The graded functors $f^{*}$ and $f_{*}$ of $\S 5.2$ are actually $H_{Y}$-graded:

Proposition 5.6.1. Let $f: X \rightarrow Y$ be a ringed-space map, and $C \in \mathbf{D}(Y)$, $D \in \mathbf{D}(Y), A \in \mathbf{D}(X)$ and $B \in \mathbf{D}(X)$.

(i) The map $f^{*}: \mathbf{E}_{Y}(C, D) \rightarrow \mathbf{E}_{X}\left(f^{*} C, f^{*} D\right)$ is $H_{Y}$-linear.

(ii) The map $f_{*}: \mathbf{E}_{X}(A, B) \rightarrow \mathbf{E}_{Y}\left(f_{*} A, f_{*} B\right)$ is $H_{Y}$-linear.

(iii) If $C=D$ (respectively $A=B$ ) then the map in (i) (respectively (ii)) is a homomorphism of graded $H_{Y}$-algebras.

Proof. (i) We need to show, for

$$
\gamma: C \rightarrow D[i] \text { in } \mathbf{E}_{Y}^{i}(C, D) \text { and } h: \mathcal{O}_{Y} \rightarrow \mathcal{O}_{Y}[n] \text { in } \mathbf{E}_{Y}^{n}\left(\mathcal{O}_{Y}, \mathcal{O}_{Y}\right)=H_{Y}^{n},
$$

that $f^{*}(\gamma h)=\left(f^{*} \gamma\right) h$-whence by symmetry, $f^{*}(h \gamma)=h\left(f^{*} \gamma\right)$. Underlying definitions show that the equality in question amounts to commutativity of the border of the next diagram (5.6.2), where the unlabeled maps are natural (see [L3, 3.2.4(i)]), and "=" represents various canonical isomorphisms.

In the subdiagrams (1) and (2) of (5.6.2) one can replace $C$ by a q-flat resolution $P_{C}$ that belongs to a family of q-flat resolutions that commute with translation (see [L3, 2.5.5], and thereby reduce the question of commutativity to the analogous one in which all derived functors are replaced by ordinary functors of complexes. The latter question is easily disposed of.

Commutativity of the other subdiagrams is straightforward to verify.

(ii) As in (i), given $\alpha: A \rightarrow B[i]$ (in $\mathbf{E}_{X}^{i}(A, B)$ ) and $h: \mathcal{O}_{Y} \rightarrow \mathcal{O}_{Y}[n]$, one wants commutativity of the border of the next diagram (5.6.3), in which $p_{2}(F, G)$ is the 
$(5.6 .2)$

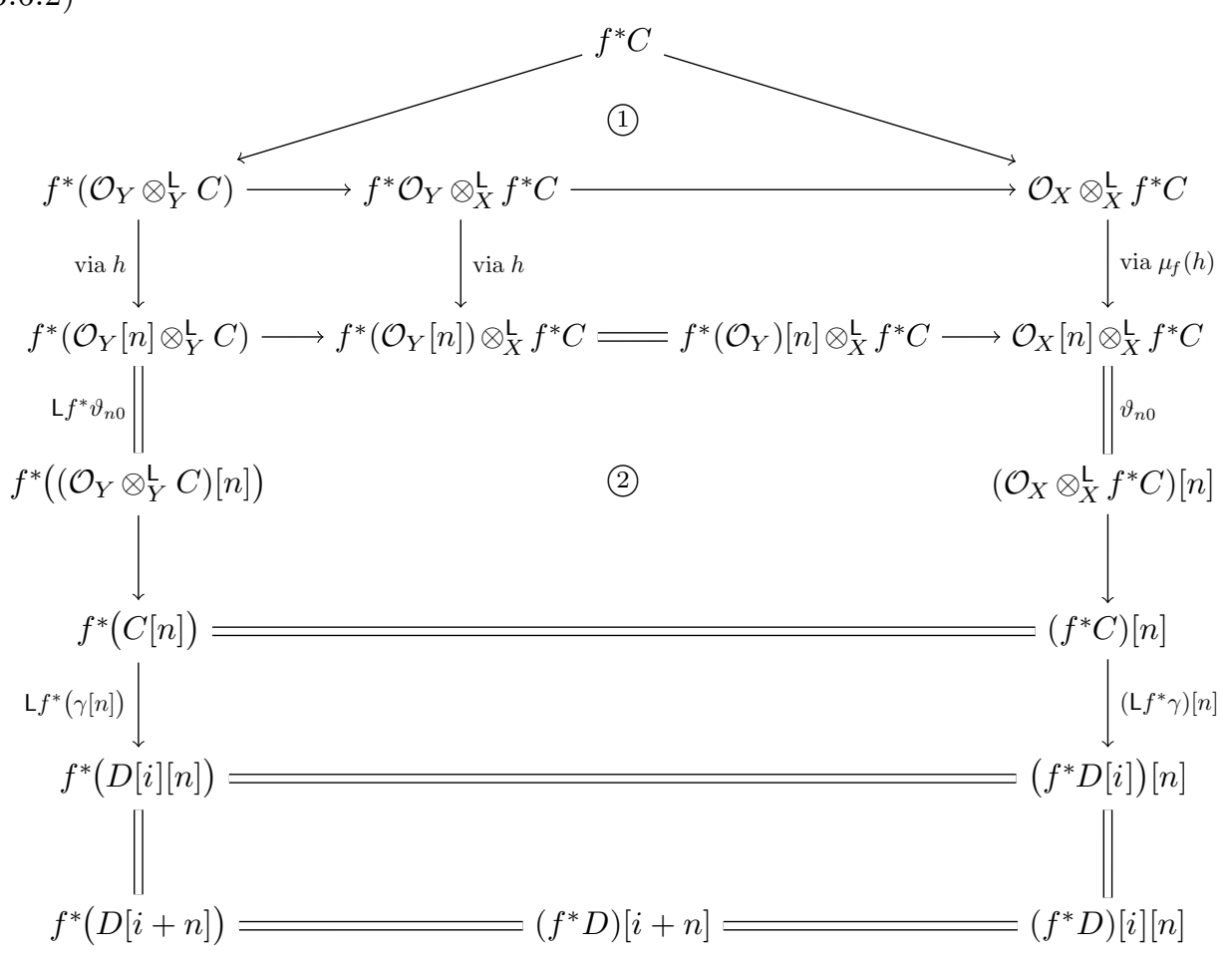

bifunctorial map adjoint to the natural composition in $\mathbf{D}(X)$

$$
f^{*}\left(F \otimes_{Y}^{\llcorner} f_{*} G\right) \rightarrow f^{*} F \otimes_{X}^{\llcorner} f^{*} f_{*} G \rightarrow f^{*} F \otimes_{X}^{\llcorner} G \quad(F, G \in \mathbf{D}(Y)) ;
$$

and where unlabeled maps are the natural ones (see [L3, 3.2.4(ii)]).

Commutativity of the unlabeled subdiagrams of (5.6.3) is easily checked.

Commutativity of subdiagram (3) is shown in [L3, p. 104].

As for (4), it suffices to prove commutativity of the adjoint diagram, namely the border of the natural $\mathbf{D}(X)$-diagram (5.6.4) below.

Diagram (5) is the commutative diagram (2) in (5.6.2), with $C=f_{*} A$.

Diagram (6) is "dual" to diagram (2) in (5.2.2), so its commutativity can be proved as indicated in the last line of the proof of Proposition 5.2.1.

Commutativity of the remaining subdiagrams is straightforward to verify.

Thus (4) commutes, and (ii) results.

(iii) This follows from (i) (respectively (ii)) and functoriality of $f^{*}$.

5.7. Recall examples (a) and (b) in $§ 5.1 .5$. These examples support a twisted inverse-image pseudofunctor $(-)_{+}^{!}$, as follows.

A scheme-map $f: X \rightarrow Y$ is essentially smooth (resp. essentially étale) if it is essentially of finite presentation (\$5.1.4) and formally smooth (resp. formally étale), i.e., for each $x \in X$, the local ring $\mathcal{O}_{X, x}$ is formally smooth (resp. formally étale) over $\mathcal{O}_{Y, f x}$ for the discrete topologies, see $\left[\mathrm{Gr}_{0}\right.$, p. 115, 19.10.2] and cf. [Gr4, §17.1 and Thm.17.6.1]. From [Gr4, Theorems (17.5.1) and (17.6.1)] it follows that any essentially smooth or essentially étale map is flat. 
$(5.6 .3)$

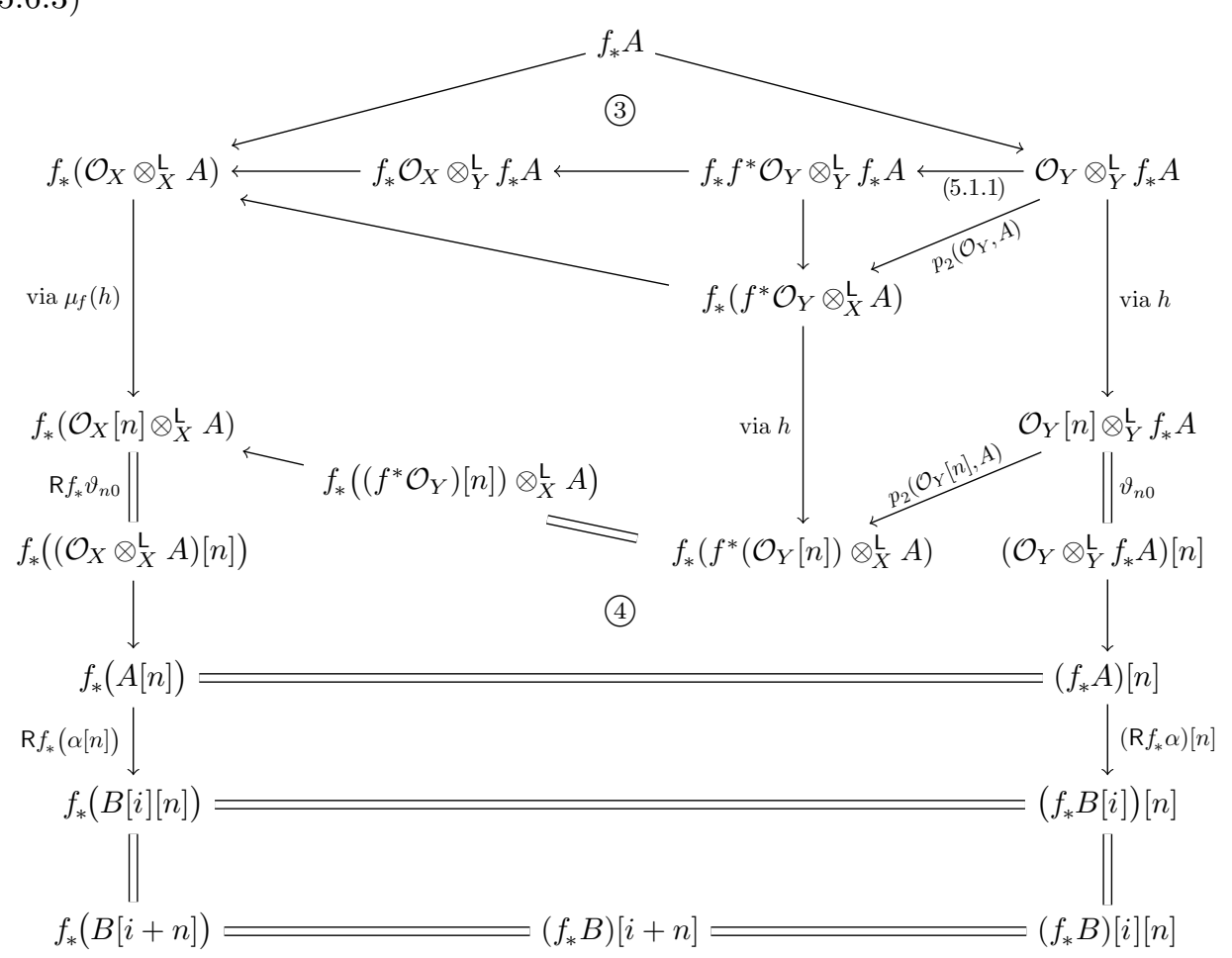

For a ringed space $X$, let $\mathbf{D}_{\mathrm{qc}}^{+}(X) \subset \mathbf{D}_{\mathrm{qc}}(X)$ be the full subcategory with objects those complexes $G \in \mathbf{D}_{\mathrm{qc}}(X)$ such that $H^{n}(G)=0$ for all $n \ll 0$.

In case (a), [Nk2, 5.3] gives a contravariant $\mathbf{D}_{\mathrm{qc}}^{+}$-valued pseudofunctor $(-)_{+}^{!}$over $\mathbf{S}$, uniquely determined up to isomorphism by the properties:

(i) When restricted to proper maps, $(-)_{+}^{!}$is pseudofunctorially right-adjoint to the right-derived direct-image pseudofunctor $\mathrm{R} f_{*}$.

Thus for proper $f: X \rightarrow Y, f_{+}^{!}$is defined on all of $\mathbf{D}_{\mathrm{qc}}(Y)$, and there is a counit map

$$
\overline{\int_{f}}: R f_{*} f_{+}^{!} \rightarrow \operatorname{id}_{\mathbf{D}_{\text {qc }}(Y)}
$$

such that (2.4.4), mutatis mutandis, commutes (cf. [L3, proof of 4.1.2]); and further, to any independent S-square $\mathbf{d}$ as in Proposition 5.2.4, there is associated the functorial isomorphism $\theta_{\mathbf{d}}: u^{*} f_{*} \stackrel{\sim}{\longrightarrow} g_{*} v^{*}$, whose restriction $\mathrm{L} u^{*} \mathrm{R} f_{*} \stackrel{\sim}{\longrightarrow} \mathrm{R} g_{*} \mathrm{~L} v^{*}$ to derived-category functors we denote by $\bar{\theta}_{\mathbf{d}}$.

There results the base-change map

$$
\overline{\mathrm{B}}_{\mathbf{d}}: v^{*} f_{+}^{!} \rightarrow g_{+}^{!} u^{*}
$$

that is adjoint to the natural composition

$$
\mathrm{R} g_{*} v^{*} f_{+}^{!} \underset{\bar{\theta}_{\mathbf{d}}^{-1}}{\stackrel{\sim}{\longrightarrow}} u^{*} \mathrm{R} f_{*} f_{+}^{!} \underset{\bar{J}_{f}}{\longrightarrow} u^{*} .
$$


$(5.6 .4)$

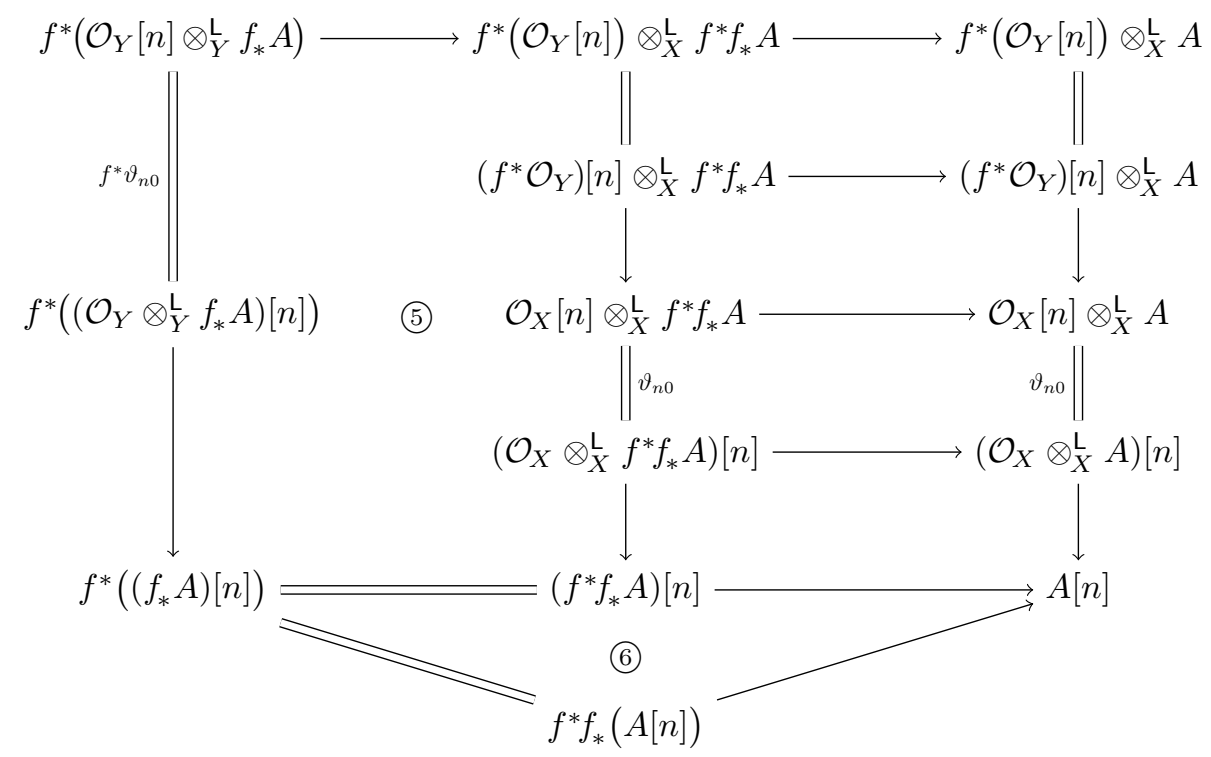

(ii) When restricted to essentially étale maps, $(-)_{+}^{!}$is equal to the usual inverseimage pseudofunctor (derived or not).

(iii) For each independent S-square $\mathbf{d}$ as in 5.2.4, with $f$ (hence $g$ ) proper and $u$ (hence $v$ ) essentially étale, $\overline{\mathrm{B}}_{\mathbf{d}}$ is the natural composite isomorphism

$$
v^{*} f_{+}^{!}=v_{+}^{!} f_{+}^{!} \stackrel{\sim}{\longrightarrow}(f v)_{+}^{!}=(u g)_{+}^{!} \stackrel{\sim}{\longrightarrow} g_{+}^{!} u_{+}^{!}=g_{+}^{!} u^{*} .
$$

There is a similarly-characterized pseudofunctor $(-)_{+}^{!}$in case (b)-argue as in [Nk1, Theorem 7.3.2], using [L3, 4.7.4 and 4.8.2.3].

The point of this subsection is to extend $(-)_{+}^{!}$to an $H_{Y}$-graded pseudofunctor $(-)^{!}$ taking values in the categories $\mathbf{D}_{W}$.

For any $f: X \rightarrow Y$ in $\mathbf{S}$, denote the "relative dualizing complex" $f_{+}^{!} \mathcal{O}_{Y}$ by $\mathcal{D}_{f}$. Recalling from $\S 5.2$ that we write $f^{*} C$ for $\mathrm{L} f^{*} C$, and with $\bar{\otimes}$ as in (5.4.1), set

$$
f^{!} C:=\mathcal{D}_{f} \bar{\otimes} f^{*} C \quad\left(C \in \mathbf{D}_{Y}\right) .
$$

It follows from Propositions 5.5 and 5.6.1(i) that $f^{!}(-)$is an $H_{Y}$-graded functor from $\mathbf{D}_{Y}$ to $\mathbf{D}_{X}$.

Next, for any $X \stackrel{f}{\rightarrow} Y \stackrel{g}{\rightarrow} Z$ in S, we need a degree-0 functorial isomorphism $\mathrm{ps}^{!}: f^{!} g^{!} \stackrel{\sim}{\longrightarrow}(g f)^{!}$.

By $[\mathrm{Nk} 2,5.8]$ (in case (a)), or by [L3, 4.7.2] (in case (b)), there is a canonical functorial isomorphism

$$
\chi_{C}^{f}: \mathcal{D}_{f} \otimes_{X}^{\llcorner} f^{*} C \stackrel{\sim}{\longrightarrow} f_{+}^{!} C \quad\left(C \in \mathbf{D}_{\mathrm{qc}}^{+}(Y)\right) .
$$

There is, in particular, an isomorphism

$$
\chi_{\mathcal{D}_{g}}^{f}: \mathcal{D}_{f} \otimes_{X}^{\llcorner} f^{*} \mathcal{D}_{g} \stackrel{\sim}{\longrightarrow} \mathcal{D}_{g f} .
$$


We can now define a degree-0 functorial isomorphism

$$
\mathrm{ps}^{!}: f^{!} g^{!} E \stackrel{\sim}{\longrightarrow}(g f)^{!} E \quad\left(E \in \mathbf{D}_{Z}\right)
$$

to be the natural functorial composite

$$
\mathcal{D}_{f} \otimes_{X}^{\llcorner} f^{*}\left(\mathcal{D}_{g} \otimes_{Y}^{L} g^{*} E\right) \stackrel{\sim}{\longrightarrow}\left(\mathcal{D}_{f} \otimes_{X}^{L} f^{*} \mathcal{D}_{g}\right) \otimes_{X}^{L} f^{*} g^{*} E \stackrel{\sim}{\longrightarrow} \mathcal{D}_{g f} \otimes_{X}^{L}(g f)^{*} E .
$$

By the proof of $[\mathrm{L} 3,4.9 .5]$, when $E \in \mathbf{D}_{\mathrm{qc}}^{+}(Z)$, this ps! can be identified via $\chi_{g^{!} E}^{f}, \chi_{E}^{g}$ and $\chi_{E}^{g f}$ with the isomorphism given by $\mathrm{ps}_{+}^{!}: f_{+}^{!} g_{+}^{!} \stackrel{\sim}{\longrightarrow}(g f)_{+}^{!}$.

Furthermore, for any $X \stackrel{f}{\rightarrow} Y \stackrel{g}{\rightarrow} Z \stackrel{h}{\rightarrow} W$ in S, the following natural diagram commutes,

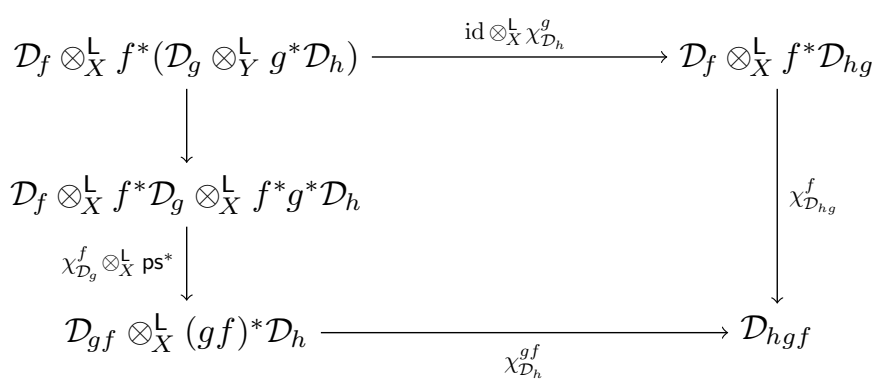

since it is isomorphic to the natural diagram

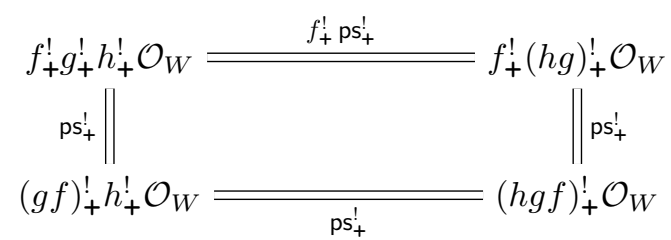

which commutes because $(-)_{+}^{!}$and $\mathrm{ps}_{+}$form a pseudofunctor.

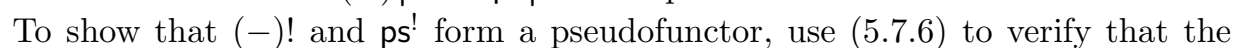
following expansion (5.7.7) of the second diagram in (2.2.1) commutes.

To see that subdiagram (1) commutes when applied to, say, $E \in \mathbf{D}(W)$, replace $\mathcal{D}_{g}, g^{*} \mathcal{D}_{h}$ and $g^{*} h^{*} E$ by q-flat resolutions to reduce to the analogous question for ordinary complexes and nonderived tensor products, which is now easily settled.

Similarly, for commutativity of (2) replace $\mathcal{D}_{h}$ and $h^{*} E$ by q-flat resolutions, and argue as in the middle of [L3, p. 124].

Checking commutativity of the remaining subdiagrams is straightforward.

5.8. Consider, in $\mathrm{S}$, an independent square

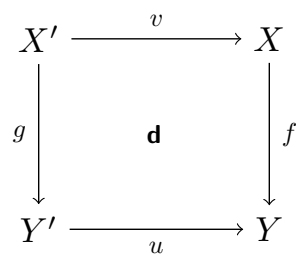

By Proposition 5.2.4, the associated map $\theta_{\mathbf{d}}: u^{*} f_{*} \rightarrow g_{*} v^{*}$ is an isomorphism. 


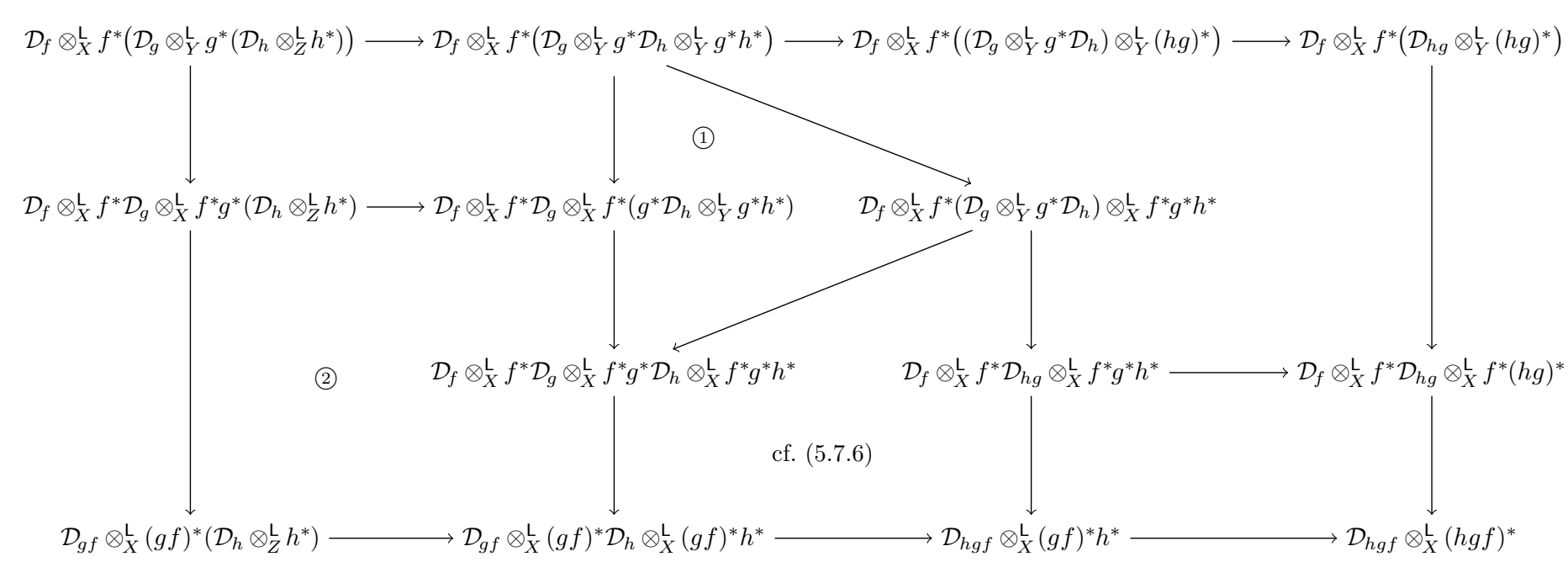


5.8.2. With notation as in (5.7.1), the functorial flat base-change isomorphism

$$
\overline{\mathrm{B}}_{\mathbf{d}}(G): v^{*} f_{+}^{!} G \rightarrow g_{+}^{!} u^{*} G \quad\left(G \in \mathbf{D}_{\mathrm{qc}}^{+}(Y)\right)
$$

is defined in case (a) of $\S 5.1 .5$ as follows.

If $f$ (hence $g$ ) is proper, then $\bar{B}_{\mathbf{d}}$ is, as in (5.7.2), the $\mathbf{D}\left(X^{\prime}\right)$-map adjoint to the composite map

$$
g_{*} v^{*} f_{+}^{!} \stackrel{\bar{\theta}_{\mathrm{d}}^{-1}}{\longrightarrow} u^{*} f_{*} f_{+}^{!} \stackrel{u^{*} \bar{f}_{f}}{\longrightarrow} u^{*} .
$$

That in this case $\bar{B}_{\mathbf{d}}(G)$ is an isomorphism for all $G \in \mathbf{D}_{\mathrm{qc}}^{+}(Y)$ is a basic fact of Grothendieck duality theory [L3, Corollary 4.4.3], [Nk2, Theorem 5.3].

When $f$ is not necessarily proper, there exists a factorization $f=\bar{f} \circ f$ with $\bar{f}$ proper and $f$ a localizing immersion [Nk2, Theorem 4.1]. Localizing immersions are set-theoretically injective maps that on sufficiently small affine sets correspond to localization of rings. They are flat monomorphisms, and if of finite type, open immersions, see $[\mathrm{Nk} 2,2.7,2.8 .8,2.8 .7,2.8 .3]$. They are essentially étale, so $f_{+}^{!}=f^{*}$. Localizing immersions remain so after base change [Nk2, 2.8.1]. Hence $\mathbf{d}$ decomposes into two fiber squares

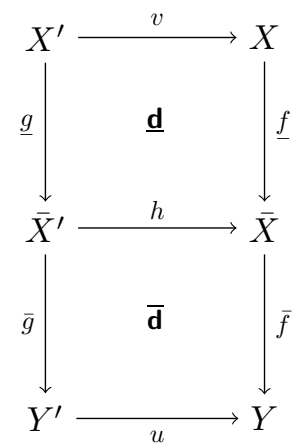

where $\underline{g}$ is a localizing immersion, so that $\underline{g}^{!}=\underline{g}^{*}$.

Let $\bar{B}(\overline{\mathbf{d}}, \underline{\mathbf{d}})$ be the composite isomorphism, in $\mathbf{D}\left(X^{\prime}\right)$,

$$
v^{*} f_{+}^{!} \stackrel{\sim}{\longrightarrow} v^{*} \underline{f}_{+}^{!} \bar{f}_{+}^{!}=v^{*} \underline{f}^{*} \bar{f}_{+}^{!} \stackrel{\underline{\mathrm{ps}}}{=} \underline{g}^{*} h^{*} \bar{f}_{+}^{!} \underset{\overline{\mathrm{B}}_{\overline{\mathbf{d}}}}{\stackrel{\sim}{\longrightarrow}} \underline{g}^{*} \bar{g}_{+}^{!} u^{*}=\underline{g}_{+}^{!} \bar{g}_{+}^{!} u^{*} \stackrel{\mathrm{ps} !}{=} g_{+}^{!} u^{*} .
$$

Arguing as in the proof of [L3, Theorem 4.8.3], one shows that $\bar{B}(\overline{\mathbf{d}}, \underline{\mathbf{d}})$ depends only on $\mathbf{d}$, and not on its decomposition. We may therefore denote this functorial isomorphism simply by $\bar{B}_{\mathbf{d}}$. (See also $[\mathrm{Nk} 2,5.2,5.3]$.)

In particular, we have the $\mathbf{D}\left(X^{\prime}\right)$-isomorphism

$$
\overline{\mathrm{B}}_{\mathbf{d}}\left(\mathcal{O}_{Y}\right): v^{*} \mathcal{D}_{f}=v^{*} f_{+}^{!} \mathcal{O}_{Y} \stackrel{\sim}{\longrightarrow} g_{+}^{!} u^{*} \mathcal{O}_{Y}=\mathcal{D}_{g} .
$$

Case (b) of $\S 5.1 .5$ can be treated analogously, see [Nk1, Theorem 7.3.2(2)].

5.8.4. Now, referring to (5.8.1), we define the $\mathbf{D}_{X^{\prime}}$-isomorphism

$$
\mathrm{B}_{\mathbf{d}}(G): v^{*} f^{!} G \rightarrow g^{!} u^{*} G \quad\left(G \in \mathbf{D}_{Y}\right)
$$


to be the natural composition

$$
v^{*}\left(\mathcal{D}_{f} \bar{\otimes} f^{*} G\right) \stackrel{\sim}{\longrightarrow} v^{*} \mathcal{D}_{f} \bar{\otimes} v^{*} f^{*} G \underset{(5.8 .3)}{\stackrel{\sim}{\longrightarrow}} \mathcal{D}_{g} \bar{\otimes} v^{*} f^{*} G \stackrel{\mathrm{id} \bar{\otimes} \mathrm{ps}^{*}}{=} \mathcal{D}_{g} \bar{\otimes} g^{*} u^{*} G
$$

It results from [L3, Exercise 4.9.3(c)] that if $G \in \mathbf{D}_{\mathrm{qc}}^{+}(Y)$ then

$$
\mathrm{B}_{\mathbf{d}}(G)=\overline{\mathrm{B}}_{\mathbf{d}}(G)
$$

It is left to the reader to verify that $B_{\mathbf{d}}$ is a degree-0 functorial map.

It is also left to the reader to use the definition of $B_{\mathbf{d}}$ to expand the horizontal and vertical transitivity diagrams (2.3.1) and (2.3.2) and to verify that the expanded diagrams commute, using e.g., transitivity for $\bar{B}_{\mathbf{d}}$ (see [L3, p. 205, (3)] and [L3, p. 208, Theorem 4.8.3] — whose proof, in view of Nayak's compactification theorem [Nk2, Theorem 4.1], extends to essentially finite-type maps), transitivity for $\theta_{\mathbf{d}}$ (cf. [L3, Prop. 3.7.2, (ii) and (iii)]), and the "dual" [L3, pp. 106-107] of the last diagram in $[\mathrm{L} 3,3.4 .2 .2]$, as treated in the first paragraph of [L3, p. 104].

5.9. Let $f: X \rightarrow Y$ be a confined S-map (see $§ 5.1 .5$ ). We now define a degree-0 functorial map $\int_{f}: f_{*} f^{!} \rightarrow$ id that satisfies transitivity (see $\S 2.4$ ).

The projection map $p(F, G)\left(F \in \mathbf{D}_{\mathrm{qc}}(X), G \in \mathbf{D}_{\mathrm{qc}}(Y)\right)$ is the natural composition, in $\mathbf{D}_{\mathrm{qc}}(Y)$,

$$
f_{*} F \otimes_{Y}^{\mathrm{L}} G \rightarrow f_{*} f^{*}\left(f_{*} F \otimes_{Y}^{\mathrm{L}} G\right) \rightarrow f_{*}\left(f^{*} f_{*} F \otimes_{X}^{\mathrm{L}} f^{*} G\right) \rightarrow f_{*}\left(F \otimes_{X}^{\mathrm{L}} f^{*} G\right) .
$$

This $p(F, G)$ is an isomorphism [L3, 3.9.4]. Denote its inverse by $\tilde{p}(F, G)$.

From (5.7.1) we have a $\mathbf{D}_{\mathrm{qc}}(Y)$-map $f_{*} \mathcal{D}_{f} \rightarrow \mathcal{O}_{Y}$. Using this map, let $\overline{\int_{f}}(G)$ be the natural functorial composition

$$
f_{*}\left(\mathcal{D}_{f} \otimes_{X}^{\mathrm{L}} f^{*} G\right) \stackrel{\tilde{p}\left(\mathcal{D}_{f}, G\right)}{\longrightarrow} f_{*} \mathcal{D}_{f} \otimes_{Y}^{\mathrm{L}} G \longrightarrow \mathcal{O}_{Y} \otimes_{Y}^{\mathrm{L}} G \stackrel{\sim}{\longrightarrow} G
$$

LEMMA 5.9.2. This $\overline{\int_{f}}$ extends to a degree-0 map $\int_{f}$ of graded endofunctors of $\mathbf{D}_{Y}$.

Proof. Set $D:=\mathcal{D}_{f}$, and write $\otimes$ for $\otimes_{X}^{\mathrm{L}}$ or $\otimes_{Y}^{\mathrm{L}}$, as the case may be. Unwinding definitions, interpret the assertion as being that for any $\mathbf{D}_{\mathrm{qc}}(Y)$-map $\alpha: A \rightarrow B[i](i \in \mathbb{Z})$, the border of the following natural diagram commutes:

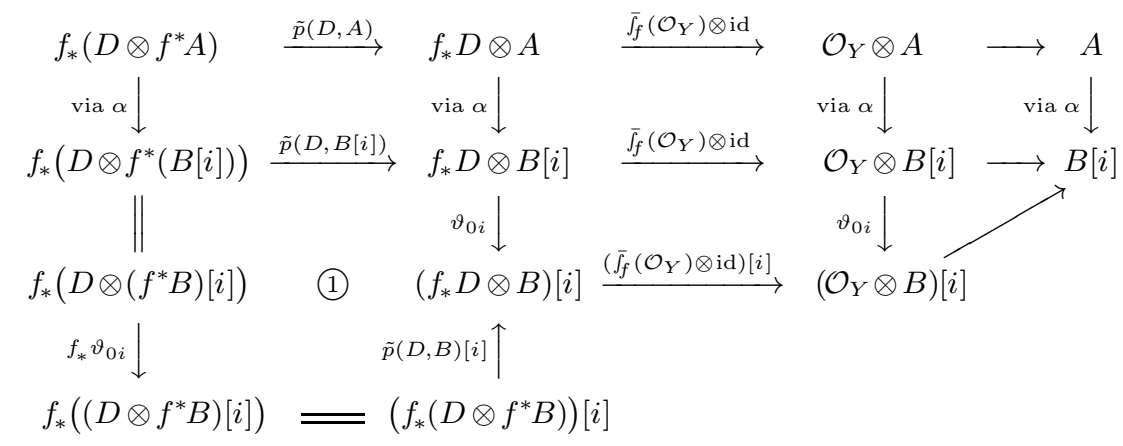


Commutativity of the unlabeled subdiagrams is evident. To prove commutativity of subdiagram (1) replace $\tilde{p}$ by $p$ (reversing the associated arrows), and then look at the $\left(\mathrm{L} f^{*} \dashv \mathrm{R} f_{*}\right)$-adjoint diagram, which is the border of the natural diagram

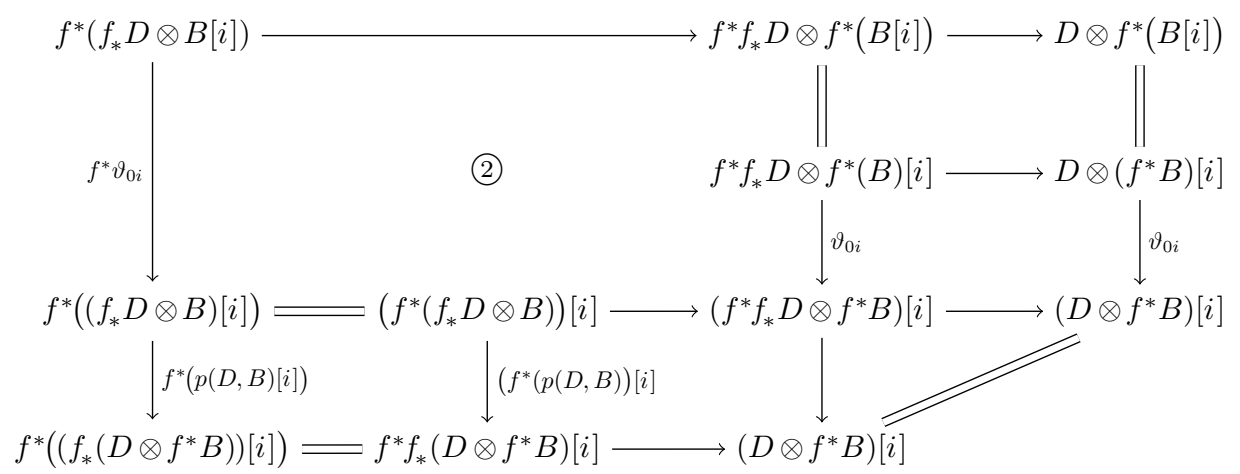

To show that subdiagram (2) commutes, replace $f_{*} D$ and $B$ by quasi-isomorphic q-flat complexes, and $\vartheta$ by $\vartheta^{\prime}$ (see 5.3.1), to reduce the question to the analogous one for ordinary complexes and nonderived functors, which situation is readily handled. Details, as well as commutativity of the other subdiagrams, are left to the reader. Thus the adjoint diagram commutes, whence so does (1), and the conclusion results. $\mathrm{C}$

Proposition 5.9.3. Let $f: X \rightarrow Y$ and $g: Y \rightarrow Z$ be confined S-maps. Then with $(-)^{!}$as in (5.7.3), $\mathrm{ps}^{!}$as in (5.7.5), and $\int$ as in 5.9.2, the transitivity diagram (2.4.4) commutes.

Proof. Global duality asserts the existence, for any S-map $f: X \rightarrow Y$, of a right adjoint $f^{\times}$for the functor $f_{*}: \mathbf{D}_{\mathrm{qc}}(X) \rightarrow \mathbf{D}_{\mathrm{qc}}(Y)$ (see [L3, 4.1]). For confined $f$, the restriction of $f^{\times}$to $\mathbf{D}_{\mathrm{qc}}^{+}(Y)$ can be identified with the functor $f_{+}^{!}$from $\S 5.7(\mathrm{i})$; in particular, the relative dualizing complex $\mathcal{D}_{f}$ in (5.7.3) can be identified with $f^{\times} \mathcal{O}_{Y}$. Furthermore, by [L3, 4.7.2 and 4.7.3(a)], $\chi_{C}^{f}$ in (5.7.4) extends to an isomorphism $f^{!} C:=\mathcal{D}_{f} \otimes_{X}^{\mathrm{L}} f^{*} C \stackrel{\sim}{\longrightarrow} f^{\times} C$ for all $C \in \mathbf{D}_{\mathrm{qc}}(Y)$; and by their very definitions, this extended $\chi_{C}^{f}$ and $\bar{\int}_{f}(C): f_{*} f^{!} C \rightarrow C$ correspond under the adjunction $\mathrm{R} f_{*} \dashv f^{\times}$.

Thus identifying $f^{!}$with $f^{\times}$via the extended isomorphism $\chi^{f}$ turns $\int_{f}$ into the counit map $\int_{f}^{\times}: f_{*} f^{\times} \rightarrow$ id. Furthermore, as in the proof of [L3, 4.9.5], that identification of $f^{!}$with $f^{\times}$turns ps! in (5.7.5) into the natural pseudofunctorial isomorphism

$$
\mathrm{ps}^{\times}: f^{\times} g^{\times} \stackrel{\sim}{\longrightarrow}(g f)^{\times}
$$

The proof of $[\mathrm{L} 3,4.1 .2]$ shows that commutativity of diagram $(2.4 .4)$ with $(-)^{\times}$, $\mathrm{ps}^{\times}$and $\int_{f}^{\times}$in place of $(-)^{!}, \mathrm{ps}^{!}$and $\int_{f}$, respectively, holds by definition of $\mathrm{ps}^{\times}$. The conclusion follows.

5.10. It remains to show that with $\mathbf{d}$ the independent square (5.8.1), diagrams (2.6.1) and (2.6.2) commute. 
5.10.1. According to the definitions in sections 5.8 and 5.9 , commutativity of (2.6.1) amounts to commutativity of the following $\mathbf{D}\left(Y^{\prime}\right)$-diagram, in which $G \in \mathbf{D}_{\mathrm{qc}}(Y), \otimes$ stands for $\otimes^{\mathrm{L}}$ with the appropriate subscript, labels on maps tell how those maps arise, and unlabeled maps are the natural ones.

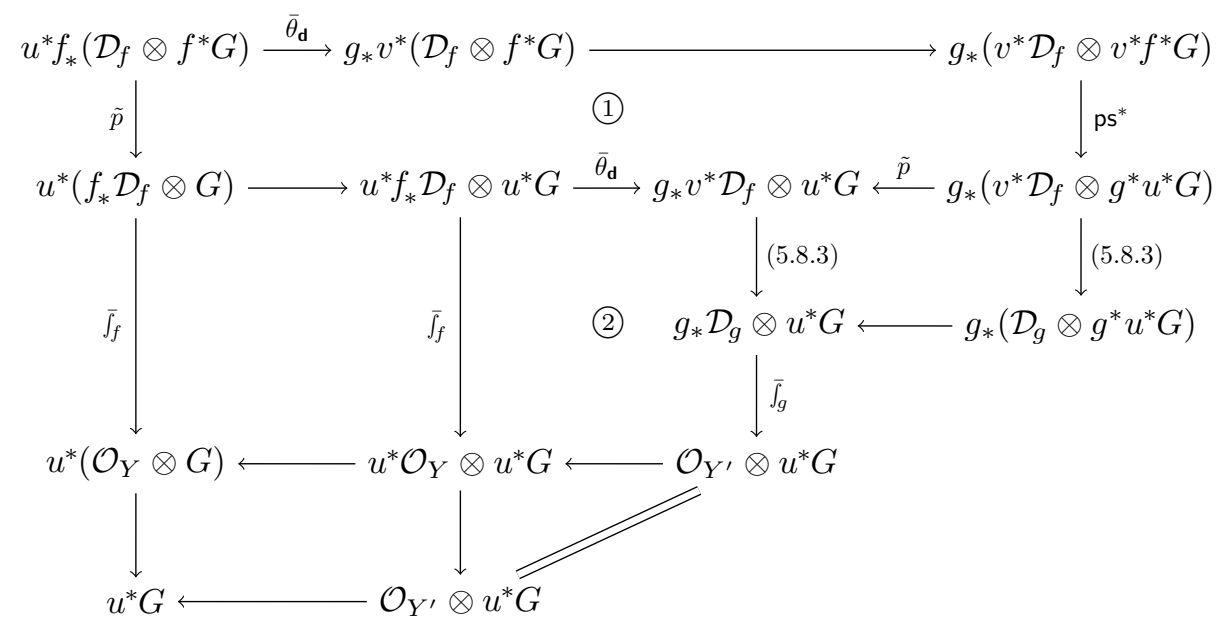

Commutativity of subdiagram (1) is given by [L3, 3.7.3].

Subdiagram (2), without $\otimes u^{*} G$, is just (2.6.1) applied to $\mathcal{O}_{Y}$. This commutes by the definition of $\bar{B}_{\mathbf{d}}\left(\mathcal{O}_{Y}\right)\left(=\mathrm{B}_{\mathbf{d}}\left(\mathcal{O}_{Y}\right)\right.$, see $\left.(5.8 .5)\right)$.

Commutativity of the remaining subdiagrams is straightforward to verify.

5.10.2. As for (2.6.2), since we are now dealing exclusively with confined maps, we may, as in the proof of Proposition 5.9.3, identify $(-)^{!}$with a right adjoint of $(-)_{*}$, and $\int_{(-)}$with the corresponding counit map.

Let $\psi_{\mathbf{d}}: v_{*} g^{!} \rightarrow f^{!} u_{*}$ be the natural composite functorial map

$$
v_{*} g^{!} \rightarrow f^{!} f_{*} v_{*} g^{!} \stackrel{\mathrm{ps}_{*}}{=} f^{!} u_{*} g_{*} g^{!} \stackrel{\int_{g}}{\longrightarrow} f^{!} u_{*} .
$$

The left adjoints of the target and source of $\psi_{\mathbf{d}}$ are then $u^{*} f_{*}$ and $g_{*} v^{*}$ respectively; and the corresponding "conjugate" map is just $\theta_{\mathbf{d}}$, cf. [L3, Exercise 3.10.4]. Since $\theta_{\mathbf{d}}$ is an isomorphism, therefore so is $\psi_{\mathbf{d}}$, and $\psi_{\mathbf{d}}^{-1}$ is the map conjugate to $\theta_{\mathbf{d}}^{-1}$ (see [L3, 3.3.7(c)]). This means that $\psi_{\mathbf{d}}^{-1}$ is the image of the identity map under the sequence of natural isomorphisms (where Hom denotes maps of functors)

$$
\begin{aligned}
& \operatorname{Hom}\left(f^{!} u_{*}, f^{!} u_{*}\right) \stackrel{\sim}{\longrightarrow} \operatorname{Hom}\left(f_{*} f^{!} u_{*}, u_{*}\right) \stackrel{\sim}{\longrightarrow} \operatorname{Hom}\left(u^{*} f_{*} f^{!} u_{*}, \text { id }\right) \\
& \underset{\operatorname{via} \theta_{\mathbf{d}}^{-1}}{\longrightarrow} \operatorname{Hom}\left(g_{*} v^{*} f^{!} u_{*}, \mathrm{id}\right) \stackrel{\sim}{\longrightarrow} \operatorname{Hom}\left(v^{*} f^{!} u_{*}, g^{!}\right) \stackrel{\sim}{\longrightarrow} \operatorname{Hom}\left(f^{!} u_{*}, v_{*} g^{!}\right) .
\end{aligned}
$$

Explicating, one gets that $\psi_{\mathbf{d}}^{-1}$ is the natural composition

$$
f^{!} u_{*} \stackrel{\eta_{v}}{\longrightarrow} v_{*} v^{*} f^{!} u_{*} \rightarrow v_{*} g^{!} g_{*} v^{*} f^{!} u_{*} \stackrel{\text { via } \theta_{\mathbf{d}}^{-1}}{\longrightarrow} v_{*} g^{!} u^{*} f_{*} f^{!} u_{*} \stackrel{\int_{f}}{\longrightarrow} v_{*} g^{!} u^{*} u_{*} \stackrel{\epsilon_{u}}{\longrightarrow} v_{*} g^{!}
$$

By the definition of $\mathbf{B}_{\mathbf{d}}$ when $g$ is proper ( $(5.8)$, it results that $\psi_{\mathbf{d}}^{-1}$ is the natural composition

$$
f^{!} u_{*} \stackrel{\eta_{v}}{\longrightarrow} v_{*} v^{*} f^{!} u_{*} \stackrel{\mathrm{B}_{\mathrm{d}}}{\longrightarrow} v_{*} g^{!} u^{*} u_{*} \stackrel{\epsilon_{u}}{\longrightarrow} v_{*} g^{!}
$$


that is, $\psi_{\mathbf{d}}^{-1}=\phi_{\mathbf{d}}$.

Commutativity of the following natural diagram, whose top row composes, by definition, to the map induced by $\psi_{\mathbf{d}}=\phi_{\mathbf{d}}^{-1}$, and whose bottom row composes to the identity, is an obvious consequence of Proposition 5.9.3. Commutativity of (2.6.2) results.

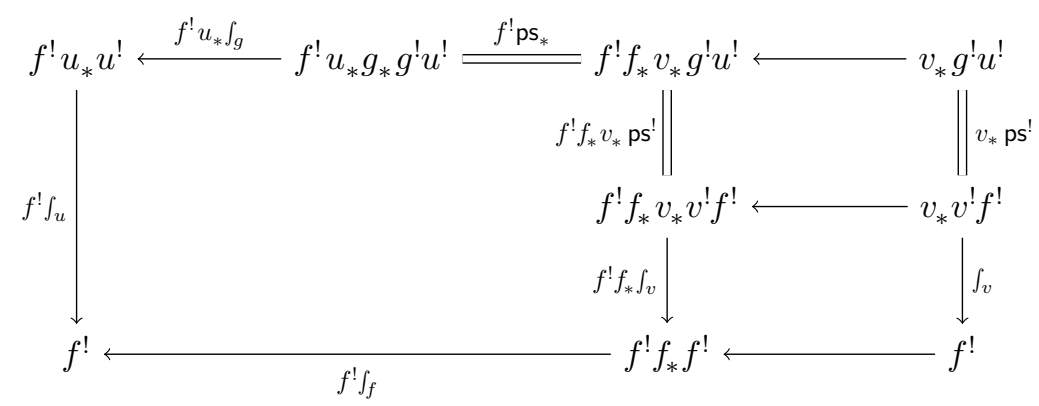

6. Example: Classical Hochschild homology of scheme-maps. This section illustrates some of the foregoing with a few remarks about earlier-known Hochschild homology and cohomology functors on schemes, especially with regard to their relation with the bivariant functors arising from Example 3.5(b). Global Hochschild theory goes back to Gerstenhaber and Schack, and has subsequently been developed by several more authors. Here we concentrate on the functors defined by Căldăraru and Willerton ([Ca1] and [CaW]).

For smooth schemes over a characteristic-zero field, bivariant homology groups coincide with classical Hochschild homology groups; but the classical Hochschild cohomology groups are only direct summands of the bivariant ones (§§6.4-6.6). Even in this special case, then, the bivariant theory has more operations on homology.

6.1. Let $f: X \rightarrow Y$ be a quasi-compact quasi-separated scheme-map, and

$$
\delta=\delta_{f}: X \rightarrow X \times_{Y} X
$$

the associated diagonal map - which is quasi-compact and quasi-separated, [Gr1, p. 294, (6.1.9)(i), (iii), and p. 291, (6.1.5)(v)].

The pre-Hochschild complex of $f$ is

$$
\mathcal{H}_{f}:=\mathrm{L} \delta^{*} \delta_{*} \mathcal{O}_{X} .
$$

(When $f$ is flat, the prefix "pre-" can be dropped, see [BF1, p. 222, 2.3.1].)

The complex $\mathcal{H}_{f}$ gives rise to classical Hochschild cohomology functors

$$
\mathcal{H H}_{X \mid Y}^{i}(F):=H^{i} \operatorname{RHom}_{X}\left(\mathcal{H}_{f}, F\right) \quad\left(i \in \mathbb{Z}, F \in \mathbf{D}_{\mathrm{qc}}(X)\right),
$$

and their global counterparts (cf. [BF1, p. 217, 2.1.1])

$$
\operatorname{HH}_{X \mid Y}^{i}(F):=\operatorname{Ext}_{X}^{i}\left(\mathcal{H}_{f}, F\right)=\mathrm{H}^{i}\left(X, \operatorname{RHom}_{X}\left(\mathcal{H}_{f}, F\right)\right) .
$$

When $X$ is affine, say $X=\operatorname{Spec}(A)$, and $Y=\operatorname{Spec}(k)$ with $k$ a field, this terminology is compatible with the classical one for $A$-modules. 
The global Hochschild cohomology

$$
\mathrm{HH}_{X \mid Y}^{*}(F):=\oplus_{i \in \mathbb{Z}} \mathrm{HH}_{X \mid Y}^{i}(F)=\operatorname{Ext}_{X}^{*}\left(\mathcal{H}_{f}, F\right)
$$

is a symmetric graded module over the commutative-graded ring

$$
H_{X}:=\oplus_{i \geq 0} \mathrm{H}^{i}\left(X, \mathcal{O}_{X}\right)
$$

see Proposition 5.4.

The sheafified version of the adjunction $L \delta^{*} \dashv \delta_{*}$ (see e.g., [L3, 3.2.3(ii)]), gives, furthermore,

$$
\begin{aligned}
\mathrm{HH}_{X \mid Y}^{i}(F) & \cong \mathrm{H}^{i}\left(X \times_{Y} X, \delta_{*} \mathrm{RHom}_{X}\left(\mathrm{~L}^{*} \delta_{*} \mathcal{O}_{X}, F\right)\right) \\
& \cong \mathrm{H}^{i}\left(X \times_{Y} X, \mathrm{RHom}_{X \times_{Y} X}\left(\delta_{*} \mathcal{O}_{X}, \delta_{*} F\right)\right) \\
& =\operatorname{Ext}_{X \times_{Y} X}^{i}\left(\delta_{*} \mathcal{O}_{X}, \delta_{*} F\right) .
\end{aligned}
$$

Proposition 6.1.1. Under Yoneda composition, the classical Hochschild cohomology associated to $f$,

$$
\operatorname{HH}_{X \mid Y}^{*}\left(\mathcal{O}_{X}\right) \cong \oplus_{i \in \mathbb{Z}} \operatorname{Hom}_{\mathbf{D}\left(X \times_{Y} X\right)}\left(\delta_{*} \mathcal{O}_{X}, \delta_{*} \mathcal{O}_{X}[i]\right)
$$

is a graded-commutative $H_{X}$-algebra, of which $H_{X}$ is a graded-ring retract.

Proof. Commutativity is well-known, cf. [BF1, §2.2]. Here is one quick way to see it. Let $\mathbf{D}_{*} \subset \mathbf{D}\left(X \times_{Y} X\right)$ be the full subcategory whose objects are the complexes $\delta_{*} G(G \in \mathbf{D}(X))$. With $p: X \times_{Y} X \rightarrow X$ the first projection, set

$$
E \otimes_{*} F:=\delta_{*}\left(p_{*} E \otimes_{X}^{\mathrm{L}} p_{*} F\right) \quad\left(E, F \in \mathbf{D}_{*}\right) .
$$

There are obvious functorial isomorphisms

$$
\lambda:\left(\delta_{*} \mathcal{O}_{X} \otimes_{*}-\right) \stackrel{\sim}{\longrightarrow} \operatorname{id}_{\mathbf{D}_{*}}, \quad \rho:\left(-\otimes_{*} \delta_{*} \mathcal{O}_{X}\right) \stackrel{\sim}{\longrightarrow} \operatorname{id}_{\mathbf{D}_{*}} .
$$

Then $\left(\otimes_{*}, \delta_{*} \mathcal{O}_{X}, \lambda, \rho\right)$ is a $\mathbb{Z}$-graded unital product, and the commutativity follows (see 1.4.3).

The $H_{X}$-algebra structure is given by 5.6 .1 (iii) (with $f$ replaced by $\delta$ ), as is a left inverse for the structure map (with $f$ replaced by $p$ ).

6.2. As in $\S 1.4 .3, \mathrm{HH}_{X \mid Y}^{*}\left(\mathcal{O}_{X}\right)$ is a graded-algebra retract of the graded center $\mathcal{C}_{*}$ of $\mathbf{D}_{*}$. There is also a natural graded-ring homomorphism from $\mathcal{C}_{*}$ to the graded center $\mathcal{C}$ of $\mathbf{D}(X)$, induced by the essentially surjective functor $p_{*}: \mathbf{D}_{*} \rightarrow \mathbf{D}(X)$. Thus there is a natural graded-ring homomorphism

$$
\varpi: \mathrm{HH}_{X \mid Y}^{*}\left(\mathcal{O}_{X}\right) \rightarrow \mathcal{C} \text {. }
$$

For flat $f$, this is canonically isomorphic to the characteristic homomorphism that plays an important role in [BF1] (where nonflat maps are also treated). It takes a $\mathbf{D}\left(X \times_{Y} X\right)$-map $\alpha: \delta_{*} \mathcal{O}_{X} \rightarrow \delta_{*} \mathcal{O}_{X}[i]$ to the natural functorial composition

$$
A \cong \mathcal{O}_{X} \otimes_{X}^{\mathrm{L}} A \cong p_{*} \delta_{*} \mathcal{O}_{X} \otimes_{X}^{\mathrm{L}} A \stackrel{\text { via } \alpha}{\longrightarrow} p_{*} \delta_{*} \mathcal{O}_{X}[i] \otimes_{X}^{\mathrm{L}} A \cong \mathcal{O}_{X}[i] \otimes_{X}^{\mathrm{L}} A \cong A[i] .
$$

One checks, for example, that in 6.1.1, the left inverse - that is induced by $p_{*}$ - for $H_{X} \rightarrow \mathrm{HH}_{X \mid Y}^{*}\left(\mathcal{O}_{X}\right)$ is the composition $\operatorname{ev}_{\mathcal{O}_{X}} \circ \varpi($ see $(1.3 .1))$. 
6.3. One has also the sheafified Hochschild homology functors

$$
\mathcal{H H}_{i}^{X \mid Y}(F):=H^{-i}\left(\mathcal{H}_{f} \otimes_{X}^{\mathrm{L}} F\right) \quad\left(i \in \mathbb{Z}, F \in \mathbf{D}_{\mathrm{qc}}(X)\right)
$$

and their global counterparts,

$$
\operatorname{HH}_{i}^{X \mid Y}(F):=\operatorname{Tor}_{i}^{X}\left(\mathcal{H}_{f}, F\right)=\mathrm{H}^{-i}\left(X, \mathcal{H}_{f} \otimes_{X}^{\mathrm{L}} F\right) .
$$

The functorial projection isomorphisms [L3, p. 139, 3.9.4]

$$
\pi(E, F): \delta_{*}\left(\delta^{*} \delta_{*} E \otimes_{X \times_{Y} X}^{\mathrm{L}} F\right) \stackrel{\sim}{\longrightarrow} \delta_{*} E \otimes_{X \times_{Y} X}^{\mathrm{L}} \delta_{*} F \stackrel{\sim}{\longrightarrow} \delta_{*}\left(E \otimes_{X}^{\mathrm{L}} \delta^{*} \delta_{*} F\right)
$$

$\left(E, F \in \mathbf{D}_{\mathrm{qc}}(X)\right)$, give, furthermore,

$$
\begin{aligned}
\mathrm{HH}_{i}^{X \mid Y}(F) & \cong \mathrm{H}^{-i}\left(X \times_{Y} X, \delta_{*}\left(\delta^{*} \delta_{*} \mathcal{O}_{X} \otimes_{X}^{\mathrm{L}} F\right)\right) \\
& \cong \mathrm{H}^{-i}\left(X \times_{Y} X, \delta_{*} \mathcal{O}_{X} \otimes_{X \times_{Y} X}^{\mathrm{L}} \delta_{*} F\right) \\
& =\operatorname{Tor}_{i}^{X \times_{Y} X}\left(\delta_{*} \mathcal{O}_{X}, \delta_{*} F\right)
\end{aligned}
$$

6.4. Căldăraru and Willerton work over a "geometric category of spaces" in which some form of Serre duality holds (see [CaW, end of Introduction]), for example, the category of smooth projective varieties over an algebraically closed field $\mathbf{k}$ of characteristic zero. What they call the Hochschild cohomology of such a variety $X$ is simply $\mathrm{HH}_{X \mid \operatorname{Spec}(\mathbf{k})}^{*}\left(\mathcal{O}_{X}\right)$.

Their Hochschild homology,

$$
\operatorname{HH}_{i}^{\mathrm{cl}}(X):=\operatorname{Hom}_{\mathbf{D}\left(X \times_{\mathbf{k}} X\right)}\left(\delta_{*} \mathcal{H} o m\left(\Omega_{X \mid \operatorname{Spec}(\mathbf{k})}^{n}[n], \mathcal{O}_{X}\right), \delta_{*} \mathcal{O}_{X}[-i]\right) \quad(i \in \mathbb{Z}),
$$

(where $n=\operatorname{dim} X$ and $\Omega_{X \mid \operatorname{Spec}(\mathbf{k})}^{n}$ is the sheaf of relative differential $n$-forms) is shown in [CaW, §4.2] to be isomorphic to the global Hochschild homology $\operatorname{HH}_{i}^{X \mid \operatorname{Spec}(\mathbf{k})}\left(\mathcal{O}_{X}\right)$. (The "cl" in the notation indicates either "Căldăraru" or "classic.") Their definitions and arguments actually apply to any essentially smooth $f: X \rightarrow Y$ (§5.7); so when such an $f$ is given we can substitute $Y$ for $\operatorname{Spec}(\mathbf{k})$ in the preceding.

6.5. Also, it is indicated near the beginning of [CaW, §5] that in their setup, Hochschild homology is isomorphic to the bivariant $\mathrm{HH}_{*}(X)$ (§3.6) associated with Example 3.5(b). This can be seen, more generally, as follows.

First, for any flat $f: X \rightarrow Y$, with $\pi_{i}: X \times_{Y} X \rightarrow X(i=1,2)$ the usual projections, and $p(-,-)$ the projection isomorphism in (5.9.1), one has, for any $F \in \mathbf{D}_{\mathrm{qc}}(X)$, the natural composite isomorphisms

$$
\begin{aligned}
\zeta_{i}(F): \delta^{*} \delta_{*} \mathcal{O}_{X} \otimes_{X}^{\mathrm{L}} F \cong \pi_{i *} \delta_{*}\left(F \otimes_{X}^{\mathrm{L}} \delta^{*} \delta_{*} \mathcal{O}_{X}\right) \\
\stackrel{\pi_{i *}\left(F, \delta_{*} \mathcal{O}_{X}\right)^{-1}}{\longrightarrow} \pi_{i *}\left(\delta_{*} F \otimes_{X}^{\mathrm{L}} \delta_{*} \mathcal{O}_{X}\right) \cong \pi_{i *}\left(\delta_{*} \mathcal{O}_{X} \otimes_{X}^{\mathrm{L}} \delta_{*} F\right) \\
\stackrel{\pi_{i *} p\left(\mathcal{O}_{X}, \delta_{*} F\right)}{\longrightarrow} \pi_{i *} \delta_{*}\left(\mathcal{O}_{X} \otimes_{X}^{\mathrm{L}} \delta^{*} \delta_{*} F\right) \cong \delta^{*} \delta_{*} F .
\end{aligned}
$$

It can be shown that the isomorphisms $\zeta_{1}$ and $\zeta_{2}$ are in fact equal.

Now suppose the map $x: X \rightarrow S$ is flat, with Gorenstein fibers. Then, as is well-known, the complex $\omega_{x}:=x ! \mathcal{O}_{S}$ is invertible, that is, each point of $X$ has a neighborhood $U$ over which the restriction of $x^{!} \mathcal{O}_{S}$ is $\mathbf{D}(U)$-isomorphic to $\mathcal{O}_{U}[m]$ for 
some $m$ (depending on $U$, but constant on any connected component of $X$ ). The complex $\omega_{x}^{-1}:=\operatorname{R} \mathcal{H} o m\left(\omega_{x}, \mathcal{O}_{X}\right)$ is also invertible, and, in $\mathbf{D}(X)$,

$$
\omega_{x} \otimes_{\mathcal{O}_{X}} \omega_{x}^{-1}=\omega_{x} \otimes_{X}^{\mathrm{L}} \omega_{x}^{-1} \cong \mathcal{O}_{X}
$$

There are natural isomorphisms

$$
\begin{aligned}
& \operatorname{HH}_{i}(X)=\operatorname{Ext}_{X}^{-i}\left(\mathcal{H}_{X}, \omega_{x}\right) \stackrel{\sim}{\longrightarrow} \operatorname{Ext}_{X}^{-i}\left(\delta^{*} \delta_{*} \mathcal{O}_{X} \otimes_{X}^{\mathrm{L}} \omega_{x}^{-1}, \mathcal{O}_{X}\right) \\
& \stackrel{\sim}{\sim} \operatorname{Ext}_{X}^{-i}\left(\delta^{*} \delta_{*} \omega_{x}^{-1}, \mathcal{O}_{X}\right) \quad(\operatorname{see}(6.5 .1)) \\
& \stackrel{\sim}{\longrightarrow} \operatorname{Ext}_{X \times_{S} X}^{-i}\left(\delta_{*} \omega_{x}^{-1}, \delta_{*} \mathcal{O}_{X}\right) \text {. }
\end{aligned}
$$

In particular, if $x$ is essentially smooth, of constant relative dimension $n$ [Nk2, 5.4], then $\omega_{x} \cong \Omega_{X \mid S}^{n}[n]$, yielding in this case that $\mathrm{HH}_{i}(X) \cong \mathrm{HH}_{i}^{\mathrm{cl}}(X)$.

6.6. For cohomology, the situation is different. Referring to Example 3.5(b), let $x: X \rightarrow S$ be the unique S-map, and $\delta: X \rightarrow X \times{ }_{S} X$ the diagonal.

There are natural functorial maps $\delta_{*} \rightarrow \delta_{*} \mathrm{~L} \delta^{*} \delta_{*} \rightarrow \delta_{*}$ composing to the identity, so the natural identifications

$$
\mathrm{HH}_{X \mid S}^{*}\left(\mathcal{O}_{X}\right) \cong \operatorname{Ext}_{X}^{*}\left(\delta_{*} \mathcal{O}_{X}, \delta_{*} \mathcal{O}_{X}\right) \text { and } \operatorname{HH}^{*}(X) \cong \operatorname{Ext}_{X}^{*}\left(\delta_{*} \mathcal{O}_{X}, \delta_{*} \mathrm{~L} \delta^{*} \delta_{*} \mathcal{O}_{X}\right)
$$

entail that the classical Hochschild cohomology $\mathrm{HH}_{X \mid S}^{*}\left(\mathcal{O}_{X}\right)$ is, as a graded group, a direct summand of the bivariant cohomology $\mathrm{HH}^{*}(X)$.

The projection $\mathrm{HH}^{*}(X) \rightarrow \mathrm{HH}_{X \mid S}^{*}\left(\mathcal{O}_{X}\right)$ can also be viewed as the map

$$
\mathrm{HH}^{*}(X)=\operatorname{Ext}_{X}^{*}\left(\mathcal{H}_{x}, \mathcal{H}_{x}\right) \rightarrow \operatorname{Ext}_{X}^{*}\left(\mathcal{H}_{x}, \mathcal{O}_{X}\right)=\operatorname{HH}_{X \mid S}^{*}\left(\mathcal{O}_{X}\right)
$$

induced by $\epsilon_{\delta}\left(\mathcal{O}_{X}\right): \mathcal{H}_{x}=\mathrm{L} \delta^{*} \delta_{*} \mathcal{O}_{X} \rightarrow \mathcal{O}_{X}$.

Since $\mathrm{HH}_{X \mid S}^{*}\left(\mathcal{O}_{X}\right)$ is graded-commutative (Proposition 6.1.1), the composition of $\varpi$ in (6.2.1) and $\mathrm{ev}_{\mathcal{H}_{x}}$ in (1.3.1) gives a natural homomorphism of graded algebras over $H_{X}$,

$$
\mathrm{HH}_{X \mid S}^{*}\left(\mathcal{O}_{X}\right) \rightarrow \mathrm{HH}_{X \mid S}^{*}\left(\mathcal{H}_{x}\right)=\mathrm{HH}^{*}(X),
$$

with image in the graded center of $\mathrm{HH}^{*}(X)$.

Thus $\mathrm{HH}^{*}(X)$ has a natural structure of graded $\mathrm{HH}_{X \mid S}^{*}\left(\mathcal{O}_{X}\right)$-algebra.

\section{REFERENCES}

[AJL] L. Alonso Tarrío, A. Jeremías Lopez and J. Lipman, Duality and flat base-change on formal schemes, Studies in duality on noetherian formal schemes and non-noetherian ordinary schemes, Contemporary Math., 244, pp. 3-90, American Math. Soc., Providence, RI, 1999. Correction: Proc. Amer. Math. Soc., 131:2 (2003), pp. 351-357.

[An] B. Angéniol, Familles de cycle algébriques-schéma de Chow, Lecture Notes in Math., 896. Springer-Verlag, Berlin-New York, 1981.

[AnL] B. Angéniol And M. LeJeune-Jalabert, Calcul différentiel et classes caractéristiques en géométrie algébrique, Travaux en Cours, 38, Hermann, Paris, 1989.

[AnZ] B. AngÉniol And F. El Zein, La classe fondamentale relative d'un cycle, Appendix to [EZ] pp. 67-93.

[BF1] R. Buchweitz And H. Flenner, Global Hochschild (co-)homology of singular spaces, Adv. Math., 217 (2008), pp. 205-242.

[BF2] The global decomposition theorem for Hochschild (co-)homology of singular spaces via the Atiyah-Chern character, Adv. Math., 217 (2008), pp. 243-281. 
[Ca1] A. CăLDĂRARU, The Mukai pairing, I: the Hochschild structure, arXiv:math/0308079.

[Ca2] The Mukai pairing, II. The Hochschild-Kostant-Rosenberg isomorphism, Adv. Math., 194 (2005), pp. 34-66.

[CaW] A. CăLdăRARU And S. Willerton, The Mukai pairing, I: a categorical approach, New York J. Math., 16 (2010), pp. 61-98.

[EZ] F. EL ZEIN, Complexe dualisant et applications á la classe fondamentale d'un cycle, Bull. Soc. Math. France Mém., 58 (1978).

[FM] W. Fulton And R. MacPherson, Categorical framework for the study of singular spaces, Mem. Amer. Math. Soc., 31 (1981), no. 243.

[GeW] S. Geller And C. Weibel, Étale descent for Hochschild and cyclic homology, Comment. Math. Helv., 66:3 (1991), pp. 368-388.

[GhS] M. Gerstenhaber And S. Schack, Algebraic cohomology and deformation theory, Deformation theory of algebras and structures and applications, pp. 11-264, NATO Adv. Sci. Inst. Ser. C Math. Phys. Sci., 247, Kluwer Acad. Publ., Dordrecht, 1988.

[Gr1] A. Grothendieck And J. A. Dieudonné, Eléments de Géométrie Algébrique I, Grundlehren Math. Wissenschaften, 166, Springer-Verlag, Berlin-New York, 1971.

[Gr4 $\left.4_{0}\right]$ Eléments de Géométrie Algébrique IV, Étude locale des schémas et des morphismes of schémas (première partie), Publications Mathématiques, 20, Institut des Hautes Études Scientifiques, Paris, 1964.

[Gr4] Eléments de Géométrie Algébrique IV, Étude locale des schémas et des morphismes of schémas (quatrième partie), Publications Mathématiques, 32, Institut des Hautes Études Scientifiques, Paris, 1967.

[HS] R. HÜBL AND P. SASTRY, Regular differential forms and relative duality, Amer. J. Math., 115:4 (1993), pp. 749-787.

[Il] L. ILlusie, Complexe cotangent et Déformations I, Lecture Notes in Math, 239, SpringerVerlag, Berlin-New York, 1971.

[Kd] M. KADDAR, Platitude géométrique et classes fondamentales relatives pondérées I, arXiv:0906.1296.

[KW] E. Kunz And R. WAldi, Regular differential forms, Contemporary Math., 79, American Math. Soc., Providence, RI, 1988.

[L1] J. LIPMAN, Dualizing sheaves, differentials and residues on algebraic varieties, Astérisque, 117 (1984).

[L2] _ Residues and traces of differential forms via Hochschild homology, Contemporary Math., 61, American Math. Soc., Providence, RI, 1987.

[L3] , Notes on derived categories and Grothendieck Duality, Lecture Notes in Math., 1960, Springer-Verlag, Berlin-New York, 2009.

[Lo] J.-L. Loday, Cyclic homology, Grundlehren Math. Wissenschaften 301, Springer-Verlag, Berlin, 1992.

[LV] W. LOWEN AND M. VAN DEN BERGH, Hochschild cohomology for abelian categories and ringed spaces, Adv. Math., 198:1 (2005), pp. 172-221.

[Nk1] S. NAYAK, Pasting pseudofunctors, Contemporary Math., 375, pp. 195-271, American Math. Soc., Providence, RI, 2005.

[Nk2] _ , Compactification for essentially finite type maps, Adv. Math., 222 (2009), pp. 527546. 
